\title{
Stress and pain in muscles and brain
}

Citation for published version (APA):

Luijcks, R. (2016). Stress and pain in muscles and brain: developing psychophysiological paradigms to examine stress and pain interactions . [Doctoral Thesis, Maastricht University]. Maastricht University. https://doi.org/10.26481/dis.20161028rl

Document status and date:

Published: 01/01/2016

DOI:

10.26481/dis.20161028rl

Document Version:

Publisher's PDF, also known as Version of record

\section{Please check the document version of this publication:}

- A submitted manuscript is the version of the article upon submission and before peer-review. There can be important differences between the submitted version and the official published version of record.

People interested in the research are advised to contact the author for the final version of the publication, or visit the DOI to the publisher's website.

- The final author version and the galley proof are versions of the publication after peer review.

- The final published version features the final layout of the paper including the volume, issue and page numbers.

Link to publication

\footnotetext{
General rights rights.

- You may freely distribute the URL identifying the publication in the public portal. please follow below link for the End User Agreement:

www.umlib.nl/taverne-license

Take down policy

If you believe that this document breaches copyright please contact us at:

repository@maastrichtuniversity.nl

providing details and we will investigate your claim.
}

Copyright and moral rights for the publications made accessible in the public portal are retained by the authors and/or other copyright owners and it is a condition of accessing publications that users recognise and abide by the legal requirements associated with these

- Users may download and print one copy of any publication from the public portal for the purpose of private study or research.

- You may not further distribute the material or use it for any profit-making activity or commercial gain

If the publication is distributed under the terms of Article $25 \mathrm{fa}$ of the Dutch Copyright Act, indicated by the "Taverne" license above, 


\section{STRESS AND PAIN IN MUSCLES AND BRAIN}

Developing psychophysiological paradigms to examine stress and pain interactions

Rosan Luijcks 


\section{STRESS AND PAIN}

IN MUSCLES AND BRAIN

Developing psychophysiological paradigms

to examine stress and pain interactions

PROEFSCHRIFT

ter verkrijging van de graad van doctor aan de Universiteit Maastricht,

op gezag van de Rector Magnificus, Prof. dr. Rianne M. Letschert, volgens het besluit van het College van Decanen,

in het openbaar te verdedigen op

vrijdag 28 oktober 2016 om 12.00 uur

door

Rosan Luijcks

Cover: Sophia Simons

○ Rosan Luijcks, Maastricht 2016

All rights reserved. No part of this publication may be reproduced or used in any manner whatsoever without prior written permission from the author. 


\section{Promotores}

Prof. dr. J.J. van Os

Prof. dr. ir. H.J. Hermens (Universiteit Twente)

Copromotor

Dr. R. Lousberg

\section{Beoordelingscommissie}

Prof. dr. F.P.M.L. Peeters (voorzitter)

Prof. dr. M.A. van den Hout (Universiteit Utrecht)

Prof. dr. E.A.J. Joosten

Dr. J.J.M.H. Oude Wansink - Strik

Prof. dr. M.R. Vollenbroek-Hutten (Universiteit Twente)

\section{Paranimfen}

Anne Marieke Wiggers

Ellis Niemantsverdriet

The research presented in this thesis was performed at the School for Mental Health \& Neuroscience (MHeNS), department of Psychiatry \& Psychology, Maastricht University, the Netherlands.

Publication of this thesis was financially supported by BrainProducts GmbH and Sint Jans Gasthuis Weert. 


\section{CONTENTS}

Chapter 1

Chapter 2 Experimentally induced stress validated by EMG activity

Chapter 3 Impact of early life adversity on EMG stress reactivity of the trapezius muscle

The influence of perceived stress on cortical reactivity: a proof-of-principle

study

Chapter 5 Processing pain: The modifying role of pain hypervigilance on trapezius muscle

$\begin{array}{ll}\text { Chapter } 6 & \text { Summary, discussion and conclusions }\end{array}$

Samenvatting

Valorization

Dankwoord

Curriculum Vitae

List of publications 
Chapter 1

Introduction

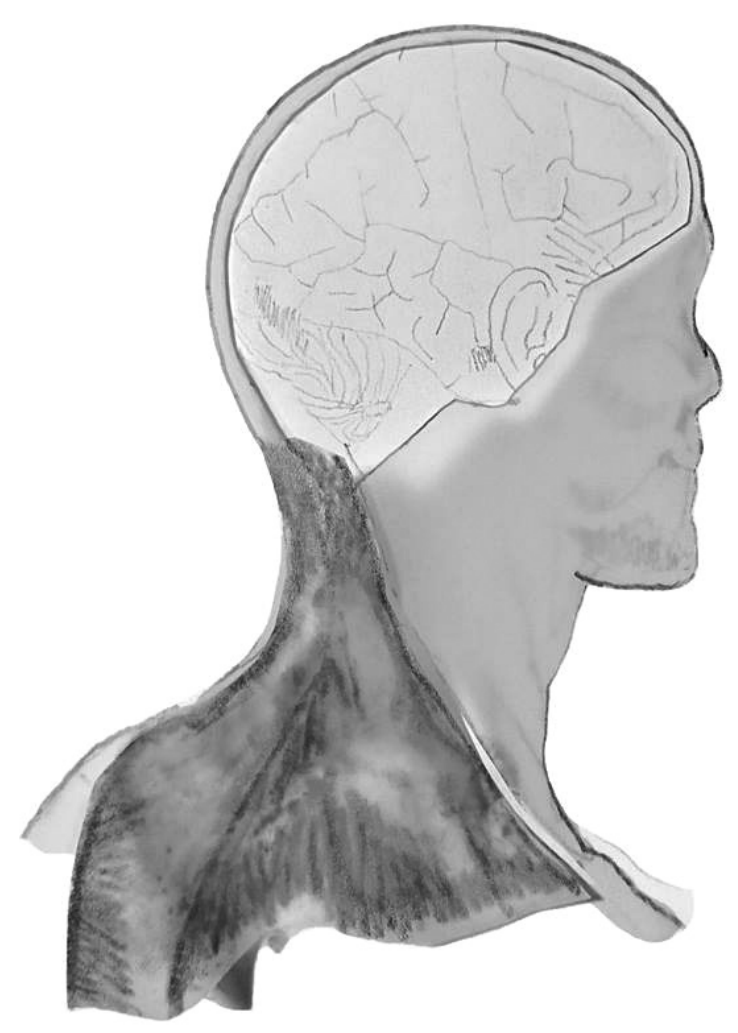




\section{BACKGROUND: EPIDEMIOLOGY AND DEFINITIONS}

Chronic pain is highly prevalent in modern society. Breivik et al. concluded that 19\% of the participants in a large European study had suffered from pain over the past six months at the time of investigation [1]. Of all people with chronic pain, a great number showed moderate to severe pain (66\% and 34\% respectively); half of them experienced constant pain. The prevalence of chronic pain in the Netherlands, as reported by Bekkering et al., correlates with these numbers, since the prevalence of moderate to severe general chronic pain among Dutch adults was estimated at 18\% [2]. According to a large-scale survey, this prevalence appears to be even higher in the United States: the point-prevalence of chronic pain (defined as chronic, recurrent, or long-lasting pain lasting for at least six months) was 31\% [3]. A large body of evidence proves that the quality of the social and working lives of people suffering from chronic pain is seriously affected [4-7]. The presence of chronic pain is distressing and frustrating for the individual and could lead to anxiousness and depression. In addition, the economic burden of chronic pain, such as health-care costs, disability and lost productivity, is most likely to be very extensive. Concrete, up-to-date data on this subject are lacking [8]

A second important health problem nowadays is psychological stress. Continuous or recurrent stress can cause stress-related health problems. These are diverse and can have an excessive impact on daily life. Mental health problems have progressively contributed to long-term disability over recent decades. In 2014, 14\% of the working population of the Netherlands experienced burnout-related complaints [9]. According to numbers published by the Ministry of Health, Welfare and Sport (Dutch: Ministerie van Volksgezondheid, Welzijn en Sport; VWS), in collaboration with ArboNed and Uitvoeringsinstituut Werknemersverzekeringen (UWV), the percentage of employee absenteeism in the Netherlands due to mental health disorders increased greatly between 2010 and 2013. For men, this share has grown from 15\% to 30\%; for women, it has grown from $25 \%$ to $41 \%$. Also, in that time, the average duration of absenteeism increased from 101 days in 2010 to 118 days in 2013 [10]. This trend is alarming. Needless to say, the consequences of stress-related health problems (such as burn-out and depression) are extensive, both on an individual level as well as for society in general.

Before setting out the main aims of this thesis, it is important to define the concepts of stress and pain. The term "stress" refers to a disturbance of homeostasis in the body or threatened disturbance of homeostasis [11]. Moreover, it is frequently used to indicate the accompanying negative emotions. The concept of stress is complex and can be viewed as an interplay of several events. Two key elements should be distinguished: first, there is a stimulus (the stressor); second, there is a stress reaction in the body, which is evoked when the stressor is perceived as a threat. In the next section more detail on the biological role of the stress reaction is provided. Next, the phenomenon of pain needs to be defined. The following definition is proposed by the Taxonomy Committee of the International Association for the Study of Pain (IASP): "Pain is an unpleasant sensory and emotional experience associated with actual or potential tissue damage, or described in terms of such damage" [12]. In origin, acute pain has a protective purpose: preventing further injury and thus enabling quick recovery. In clinical practice, however, it is generally known that there can be a discrepancy between the extent of nociceptive input and the accompanying (subjective) experience of pain. Based on this phenomenon, researchers and clinicians have agreed that pain should be viewed in a biopsychosocial model, taking into account the different aspects and modifying factors of the experience of pain (see Gatchel et al. [13])

In conclusion, when examining stress and pain a biopsychosocial approach should be considered, focusing on the biological (somatic), psychological (behavioral) and social (cultural) dimensions of these concepts [14, 15].

\section{PHYSIOLOGICAL REACTIONS DUE TO PAIN AND STRESS}

Our brain responds to both physical stressors (pain and infection), as well as to psychological stressors, whether in anticipation or in reaction. An instant systemic response to these stressors (threat) is required: both the sympathetic autonomic nervous system and the hypothalamic pituitary-adrenocortical (HPA)-axis are activated [16-18]. This induces the so-called fight-orflight reaction, resulting in an alert body state (for example, an increased heartbeat, increased muscle activity and quicker breathing). The fight-or-flight reaction was first propounded by Walter Cannon and further used by Hans Selye [19]. Selye described this reaction as being the first phase in a series of phases, which he called the general adaptation syndrome (GAS). The first phase was defined as the alarm phase, followed by the resistance phase and sometimes by the exhaustion phase, in the case of recurrent or continuous presence of stressors. In the resistance phase, the body tries to adapt to the stressor in order to regain homeostasis.

When considering this biological activity, it is important to mention two pathways of the stress response: the HPA-axis and the sympathetic-adreno-medullary (SAM) pathway, which is part of the autonomic nervous system [16, 20]. The SAM pathway reacts quite quickly in response to stressor exposure, whereas the HPA-axis functions somewhat more slowly.

A stressor can activate the HPA-axis: the hypothalamus stimulates the pituitary by releasing corticotrophin-releasing hormone (CRH). The pituitary successional produces adrenocorticotropic hormone (ACTH) which stimulates the release of glucocorticoids (in humans primarily cortisol) from the cortex of the adrenal glands [16]. These steroid hormones act on several peripheral and brain tissues. By means of a negative feedback loop, cortisol can 
regulate the secretion of $\mathrm{ACTH}$ and $\mathrm{CRH}$ by acting on the pituitary gland and the hypothalamus, thus limiting or terminating the stress response $[18,21]$. Under basal conditions, in absence of stressors, the HPA-axis operates in an episodic, circadian way, resulting in pulsatile secretion of ACTH and cortisol [22].

In the SAM system, the hypothalamus sends signals to the brain stem nucleus of the solitary tract (NST) and thus stimulates the sympathetic efflux. Adrenaline and noradrenaline are in turn excreted by the medulla of the adrenal glands and by the sympathetic nerves [16, 20]. Adrenaline in particular causes the autonomic responses present in the fight-or-flight reaction, for example, increasing the heart rate and blood pressure by exciting the cardiovascular system. The released cortisol ensures enough energy is provided for this reaction $[16,17,20]$.

\section{THE INTERACTION BETWEEN PAIN AND STRESS}

In the literature, (chronic) pain is frequently linked to stress [23-29]. Pain can be considered a physical stressor and many studies have shown the bidirectional interaction between these two concepts. Nevertheless, the exact interplay between pain and stress within this biopsychosocial context has yet to be elucidated. Epidemiologically, stress-related health problems and chronic pain are known to be highly prevalent in modern society and comorbidity often occurs [30, 31]. For example, work-related stress exposure, such as repetitive work or mental stress during work, increases the risk of stress-related pain [32-38].

When considering stress, there are essentially two types of stressors: physical and cognitive. For cognitive stressors, many object-related (such as spiders) and situation-related (such as heights, public speaking) stressors can be distinguished. Pain, as a state in which the integrity of the body and its homeostasis is threatened [39], is considered a physical stressor. The uncontrollability and unpredictability of a painful event will generate not only a pain response (i.e. reactivity), but also a state of cognitive stress (i.e. anticipatory activity) [40]. It should be noted that autonomic reactivity triggered by painful stimuli is comparable to the reactivity caused by a psychosocial stressor [41], thus emphasizing the common neurobiological pathways [16].

In case of noxious stimuli (thermal, electrical or chemical) nociceptors in the skin can respond to the potential tissue damage. Nociceptors may be subclassified: myelinated A-fibers afferents versus unmyelinated C-fibers afferents $[42,43]$. These nociceptors transmit the pain signal to the spinal cord, where it is conducted by several ascending tracts (bottom-up) [16, 43]. Upon reaching the brain, several brain regions -- i.e. the neuromatrix (including the limbic system, brain stem and somatosensory cortices) -- become involved in the processing of the pain signal, which eventually leads to the subjective experience of pain [16, 39]. Nociception can directly activate a stress response through the connections with the hypothalamus and the NST (ascending tracts), activating the HPA-system [39, 44]. It is important to note that pain perception can be modulated by both emotional and cognitive aspects, such as attention, expectations and reappraisal, as well as by the descending tracts in the spinal cord, which regulate the top-down reaction $[45,46]$.

The effect of acute stress on pain is complex, because the presence of stress can both enhance pain (hyperalgesia) and inhibit it (resulting in hypoalgesia) [47-51]. It is proposed that this effect of stress on pain could be determined by the type of stressor: severe stressors would lead to hypoalgesia, whereas a mild stressor induces a less intense emotional state and therefore may lead to hyperalgesia [52]. Furthermore, it is known that the increased cortisol levels induced by sustained activation of the stress system are related to physiological changes that can give rise to pain.

Concerning long-term effects, it is important to mention that some evidence suggests that the experience of early life stress and adverse childhood events can influence pain processing later in life. For example, they can lead to chronic headaches [53-57].

\section{From acute to chronic}

There are two sides to every coin. It is important to realize that the fight-or-flight stress response in an acute setting is fundamental and allows us to survive in the face of a challenge. However, it is the perception and appraisal of the stressor, its duration and its (un)predictability and (un)controllability which determine the effects of stress on health [40, 58-61]. This brings us to the third phase of the General Adaptation Syndrome, defined as the exhaustion phase. Stressors can be repeatedly or continuously present. Consequently, the body reaches a phase of exhaustion. Continuous and/or repetitive stress can therefore be harmful and lead to mental and physical diseases. Within this context, it is important to mention the concept of allostatic load, being the long-term result of failed adaptation or allostasis [62], resulting in pathology and chronic illness [63]. It has been demonstrated that a high allostatic load prior to adulthood (i.e. childhood trauma) is associated with a lasting impact on physiological reactivity to acute stressors later in life [64-67] and that it increases vulnerability to several disorders [68, 69], suggesting that chronic stress affects one's coping capacity. It has also been proposed that prolonged stress may lead to a vicious circle of heightened stress-reactivity and pain and may play a role in the transition process of acute to chronic pain $[13,70,71]$. In line with this theory research shows that repeated exposure to stress, accompanied by increased muscle activity, causes health care risks and could lead to skeletomuscular diseases, such as neck pain or lower back pain $[72,73]$. The development of persistent disabling lower back pain can be predicted 
by several other psychosocial factors as well, such as a poor general health status, the presence of psychiatric comorbidities, inadequate pain coping behavior [74] or catastrophizing [75].

\section{Measuring stress and pain in the context of a biopsychosocial model}

Stress, like pain, should be viewed as a theoretical construct, which cannot be measured directly. To cover the three dimensions of a biopsychosocial model, measurement of stress and pain should include both subjective measures through self-report questionnaires and objective (psycho)physiological activity. As stated above, pain should be viewed as a stressor, evoking similar reactivity of the autonomous central nervous system. Experiencing a stressor is generally accompanied by an increased level of arousal, leading to several psychophysiological

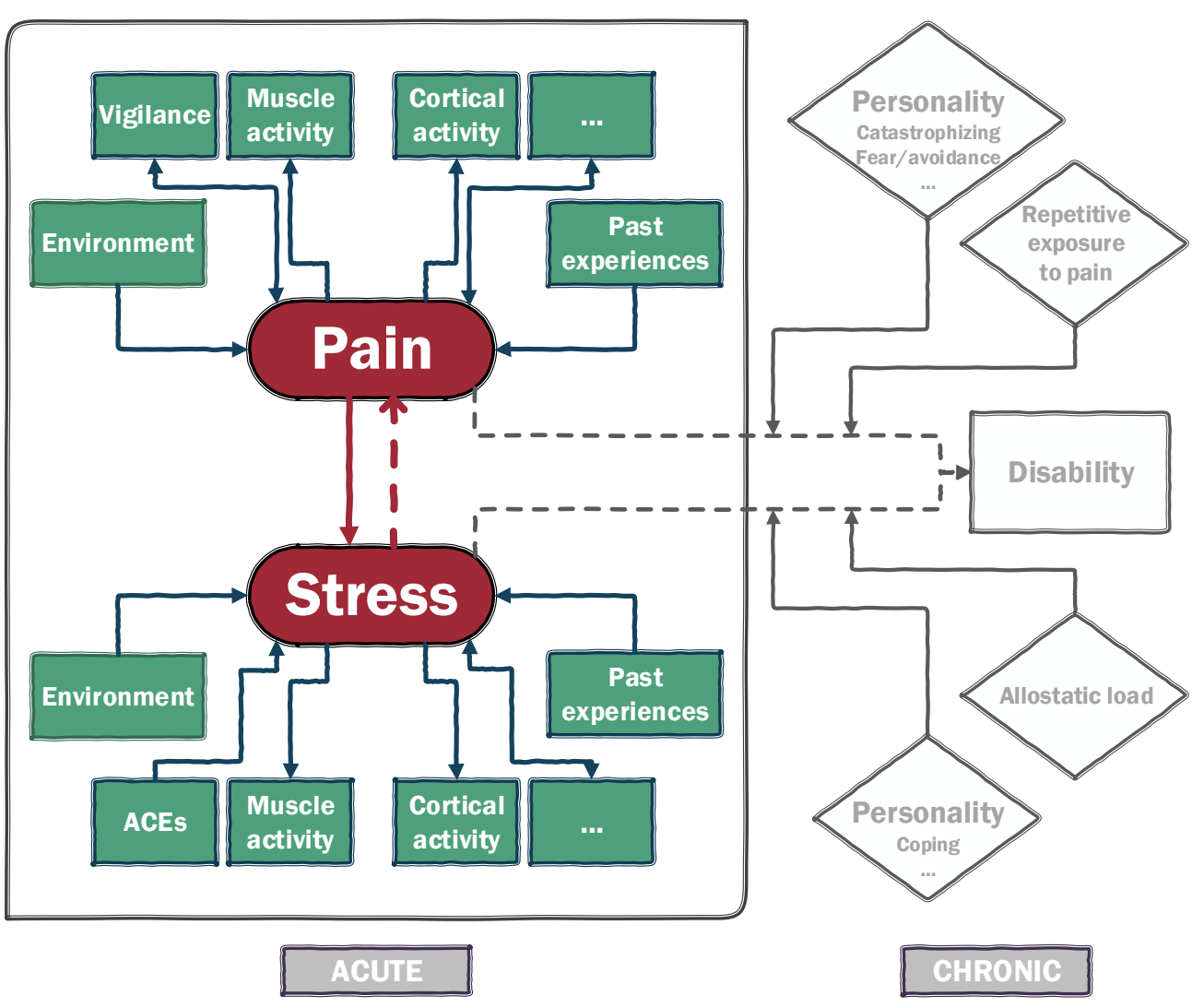

Figure 1. Biopsychosocial model of stress and pain. The acronym ACEs stands for Adverse Childhood Experiences. The left part of the model shows the acute situation, the right grey part shows the possible transition to chronicity. The arrows show the direction of the effect. The dotted lines represent potential effects, which not necessarily will occur. reactions [17]. In experimental stress research, the level of cortisol (salivary or serum) is often used as a representative of stress experienced [76]. Heart rate, heart rate variability, blood pressure and skin conductance are also used as stress representatives. In the present thesis, the focus is on electrical signals of both the brain and muscles, since alterations in these psychophysiological measures may also have clinical implications,

Figure 1 represents a biopsychosocial model of stress and pain, adapted from Turk [77] and Lazarus\&Folkman [59]. The model shows the interplay between the experience of stress and that of pain. Pain and stress are constructs, which are determined by several different factors. For both constructs, a number of factors are depicted. For example, a subject's environment can influence the experience of pain or stress. Past pain experiences and past stress experiences also influence the present experience. Both pain and stress are accompanied by enhanced psychophysiological activity such as muscle activity and cortical activity. The model also demonstrates how a phase of acute stress or acute pain can evolve into chronicity, influenced by circumstantial and personality factors.

\section{PSYCHOPHYSIOLOGICAL MEASUREMENT}

\subsection{Electroencephalography: "a window to the mind"}

As brain cells can become depolarized and electronegative due to activation of a neuron by other neurons through afferent action potentials, cortical electrical activity can be measured by using electroencephalography (EEG) [78]. Electroencephalography has a high temporal resolution, commonly measured in milliseconds, allowing us to investigate fluctuations in cortical activity. Amplitudes of EEG normally range from $10 \mu \mathrm{V}$ to $50 \mu \mathrm{V}$ in adults [79, 80]. The raw EEG data are transformed by means of a Fast Fourier Transformation, resulting in frequency bands. Delta activity, ranging from 1 to $4 \mathrm{~Hz}$, is generally an inhibitory rhythm and is typically linked with sleep in healthy humans. Theta activity ranges from 4 to $8 \mathrm{~Hz}$ and is associated with decreased alertness when observed throughout the scalp, but also with focused attention and mental effort when observed in the frontal midline. The alpha band, which ranges from 8 to $13 \mathrm{~Hz}$, is associated with relaxed wakefulness. In the posterior regions, alpha activity can be provoked by closing the eyes. Beta activity lies within the 13 to $30 \mathrm{~Hz}$ frequency range. It is linked with attention and typically replaces alpha activity during cognitive tasks. The gamma rhythm, ranging from 30 to $50 \mathrm{~Hz}$, is related to mental effort and various demanding mental processes $[79,80]$. Since it is known that cortical activity is strongly influenced by age (e.g. Dustman et al. [81]), statistical analyses are typically performed with age as a confounding variable. Note that the spatial resolution of the EEG is low. In other words, EEG measurements with a relatively small number of electrodes $(<32)$ do not give reliable information on the source of electrical activation. 
Several studies have investigated the associations between EEG activity and stress [82-86]. Within these lines of research there has been a focus on frontal alpha activity and its left and right hemisphere asymmetry $[82,84,87,88]$. Most studies report a relative decrease in alpha activity in stressful situations when compared with relaxation $[82,84,87,89]$. Additionally, it is assumed that the processing of negative emotions is linked to the right hemisphere, whereas the left hemisphere is associated with positive emotions [88]. Next to these changes in alpha activity, it is proposed that stressful conditions, such as anxiety disorders, are associated with changes in EEG gamma activity [90, 91].

As explained in the section on biological activity in the process of pain, several regions of the brain are involved in the processing of pain. In this line of research, a relationship between pain and specific cortical reactivity as measured by EEG is often reported [92-94]. It was decided not to treat this subject, however, since it was not part of the content of this thesis. A fellow member of our research group broadly discusses this subject in several studies [95, 96].

In this thesis, we focus on cortical reactivity caused by cognitive stress, by inducing stress experimentally and measuring EEG activity simultaneously.

\subsection{Electromyography}

In addition to cortical reactivity in stress experiments, as a central correlate to stress, muscle activity can be measured with electromyography (EMG), as a peripheral correlate to stress. An increase in EMG activity during stressful events is reported in most studies [97-106]. This increased EMG activity emerges particularly in the trapezius muscles [102, 107-109] and especially if the stressor is personally relevant [110]. It is interesting to examine the relationship between this altered stress-reactivity due to experiences in the past and the development/ occurrence of stress and pain-related health problems, such as chronic pain syndromes or functional somatic syndromes. For example, evidence suggests a relationship between augmented muscle activity and fibromyalgia [107]. Similarly, as with EEG, EMG is measured with a high temporal resolution $(1000 \mathrm{~Hz})$. The statistical analyses, however, are based on rootmean-square transformed data [111].

\section{EXPERIMENTAL DESIGN}

In this thesis, two experimental designs are used. Chapters 2 to 4 show studies based on a newly developed stress experiment, whereas the study in Chapter 5 is based on a pain habituation experiment. The reasoning behind the set-up of these designs is described in sections 5.1 and 5.2, below.

\subsection{Stress experiment}

The experimental manipulation of a stressor is difficult and standardization is not often achieved $[76,112]$. Since the experiment was set up to examine not only muscle activity but also cortica activity, a stress task without additional mental tasks or demands seemed to be the best fit

A few pillars or characteristics should be identified as the foundation of this experiment, introducing a stressor with a dual nature (cognitive and physical) thus enables the investigation of both forms of stress [113], and to include the aspects of unpredictability and uncontrollability [40]. The paradigm should also be simple to carry out and be independent of educational level, motivation, illness status and other factors. A novel stress experiment was developed to conform with these characteristics. This experiment is illustrated in figure 2 below. It introduces a dual stressor, consisting of the announcement of imminent receipt of a single uncontrollable and unpredictable electric shock, followed by the nociceptive stimulus.

Within this experiment, three phases can be distinguished: (i) an anticipatory pre-stimulus phase during which subjects anticipate an electric shock in a relatively uncontrolled/unpredictable situation; (ii) an immediate post-stimulus phase, showing the initial response to the nociceptive stimulus; and (iii) a return to baseline phase, which can be described as achieving a comparable level of baseline physiological activity. Based on this paradigm, stress-reactivity can be quantified, reflecting individual differences in stress susceptibility. The clear advantage of this paradigm is that the presentation of the physical stimulus has two functions: it makes the examination of a physical stressor possible, and it allows us to demarcate the period of mental stress induction, since subjects were instructed they would receive only one physical stimulus.

\section{Psychophysiological reactivity}

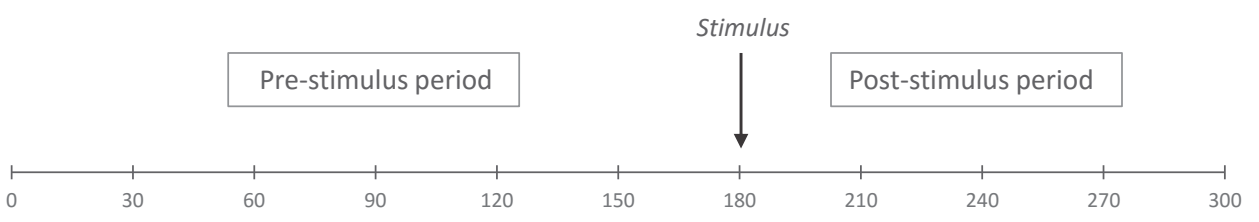

Time (in seconds)

Figure 2. Schematic illustration of the stress experiment. The total duration of the experiment is five minutes. Subjects were unaware of the intensity and timing of the electrical stimulus. The three minute pre-stimulus period was defined as the anticipatory stress phase, followed by the two minute poststimulus phase, in which a return to baseline can take place. 


\subsection{Pain habituation experiment}

In order to examine the concept of pain, a pain habituation experiment was conducted, based on earlier developed and validated studies [114-116]. Subjects received a series of electrical stimuli on the middle finger of the left hand. Subjects were unaware of the number of stimuli and had been told the stimuli would be related to the pain threshold, but could differ in intensity. The inter-stimulus intervals could also vary a little, they were told. In fact, all 25 stimuli were of the same intensity. This stimulus intensity was determined using the subject's personal sensitivity threshold and pain threshold, as described in paragraph 4.1. The interstimulus intervals were randomized between 9 and 11 seconds, to minimalize predictability

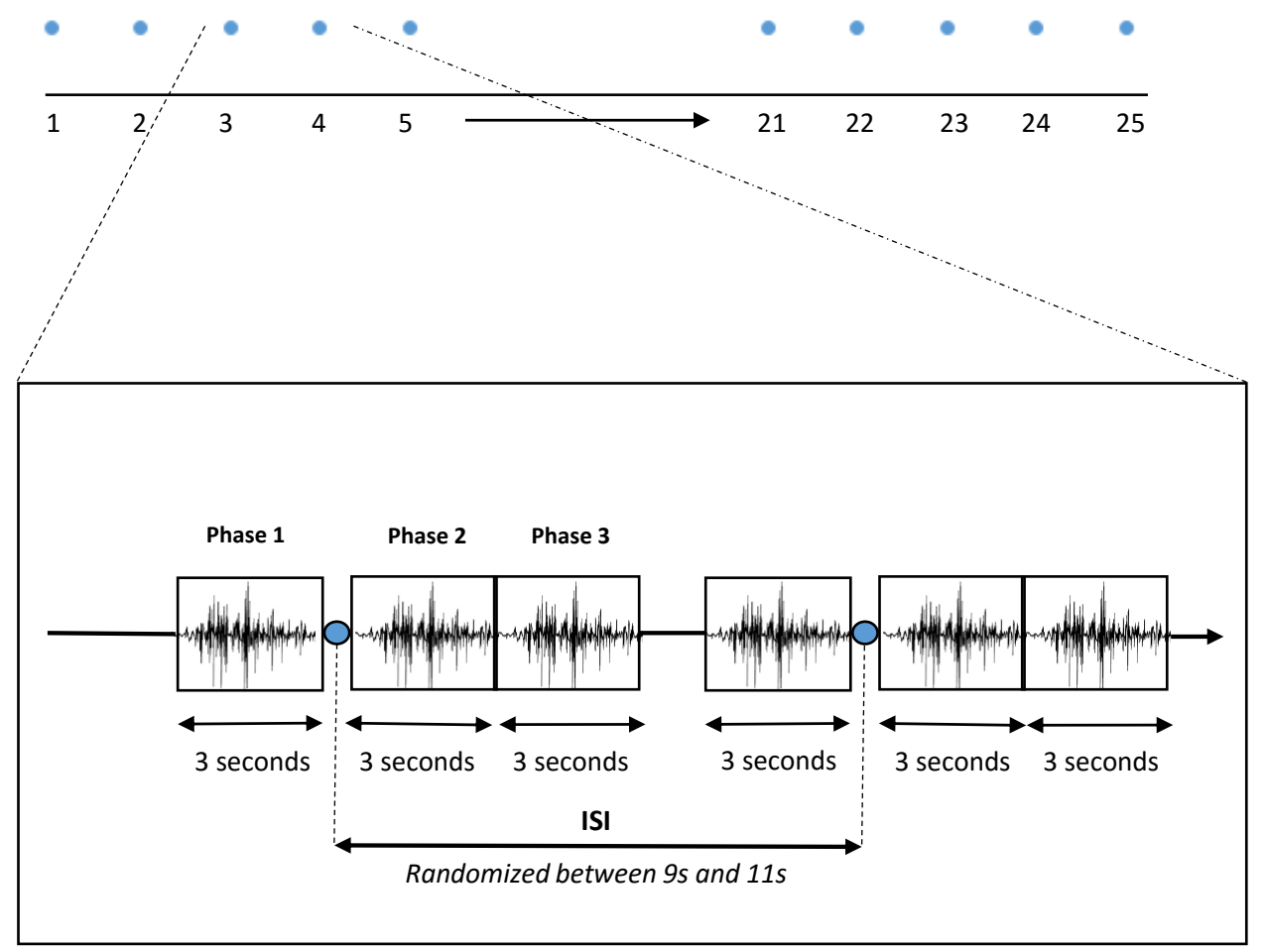

Figure 3. Schematic illustration of the pain habituation experiment. The experimental design included 25 consecutive electrical stimuli, with a variable inter-stimulus interval (randomized between 9 and 11 seconds). The subjects were unaware of the fact that all electrical stimuli were of the same intensity level. EMG activity of the left and right trapezius muscles was measured. Offline data processing allowed for the distinction of three phases: phase 1, the anticipatory phase (three seconds prior to the stimuli); phase 2, the post-stimulus pain reactivity phase (three seconds directly following the stimuli); and phase 3 , the intermediate phase (three to six seconds after the stimuli).
Subjects were asked to rate each stimulus on a numerical rating scale (NRS). For standardization purposes, the first stimulus should be rated as 60 , on a scale of 0 to $100(0=$ no pain, $100=$ strongest pain imaginable). Subjects were told to keep both hands on the table with their palms down, and not to close their eyes during the whole measurement period. A schematic illustration of this experiment can be viewed in figure 3, below.

\section{STATISTICAL METHODS}

Both EMG and EEG activity are typically measured by a computed average over a complete experimental condition. To this end, linear regression or analysis of variance (ANOVA) analyses are used. Measuring EMG over time, however, implies a hierarchical structure of the data in which consecutive time elements are nested within subjects. This hierarchical structure needs to be taken into account by using multilevel random regression procedures [117]. Intuitively, natural responses are not likely to be solely linear. In order to investigate psychophysiological measurements, both linear and non-linear time courses should be modeled. To do so multilevel regression analyses can be used. 


\section{AIMS AND OUTLINE OF THIS THESIS}

The overall aim of the work presented in this thesis is to provide more insight into stress-pain mechanisms, using psychophysiological measures. Both the natural muscle responses and the cortical reactivity in anticipation of, or caused by, a stressful or painful event are examined. The modification of psychological issues is also investigated in the context of a biopsychosocia stress vulnerability model. This led to the following research topics, presented in this thesis:

In Chapter 2, the newly developed stress paradigm is validated using EMG activity of the trapezius muscles as the dependent variable. Linear and non-linear time courses of EMG activity are examined using multilevel modeling, proving that this statistical technique provides us with additional information on the mechanics of EMG activity in a stressful experiment.

In Chapter 3, the same stress paradigm is used to study the influence of exposure to early life adversity on EMG reactivity in the stress experiment, focusing on the anticipatory stress phase.

In Chapter 4, the stress paradigm is used once more, this time examining cortical activity nstead of analyzing the averaged EEG activity pre- and post-stimulus, the linear time course of cortical activity is examined as a proof-of-principle. In addition, the influence of perceived stress on this cortical activity is demonstrated, justifying a biopsychosocial model of stress. The results of this study show that the modifying influence of perceived stress on cortical activity can be objectified.

In Chapter 5, a pain habituation experiment is used to investigate the EMG time courses, both in anticipation of a painful stimulus as well as during the pain reactivity phase. The influence of pain vigilance on this anticipatory stress activity and pain reactivity is examined to obtain more insight into the link between hypervigilance, altered EMG activity and chronic pain.

In Chapter 6, a brief summary of the findings is presented. Further discussion of possible theoretical backgrounds is presented. Finally, the implications of the findings, and directions for future research, are presented.

\section{REFERENCES}

Breivik, H., et al, Survey of chronic pain in Europe: prevalence, impact on daily life, and treatment. Eur J Pain, 2006. 10(4): p. 287-333.

2. Bekkering, G.E., et al., Epidemiology of chronic pain and its treatment in The Netherlands. Neth J Med, 2011. 69(3): p. 141-53.

3. Johannes, C.B., et al., The prevalence of chronic pain in United States adults: results of an Internet-based survey. J Pain 2010. 11(11): p. 1230-9.

4. Schrier, E., et al., Quality of life in rehabilitation outpatients: normal values and a comparison with the general Dutch population and psychiatric patients. Qual Life Res, 2015.

5. Bjornsdottir, S.V., S.H. Jonsson, and U.A. Valdimarsdottir, Mental health indicators and quality of life among individuals with musculoskeletal chronic pain: a nationwide study in Iceland. Scandinavian journal of rheumatology, 2014. 43(5): p. 419-23.

6. Lin, R.-F., et al., Correlations between quality of life and psychological factors in patients with chronic neck pain. The Kaohsiung journal of medical sciences, 2010. 26(1): p. 13-20

7. Laursen, B.S., et al., Health related quality of life and quantitative pain measurement in females with chronic nonmalignant pain. European journal of pain (London, England), 2005. 9(3): p. 267-75.

8. Turk, D.C., H.D. Wilson, and A. Cahana, Treatment of chronic non-cancer pain. Lancet, 2011. 377(9784): p. 2226-35.

9. W.E. Hooftman, G.M.J.M., B. Janssen, E.M.M. de Vroome, S.N.J. van den Bossche, Nationale Enquête Arbeidsomstandigheden 2014: methodologie en globale resultaten. Centraal Bureau voor de Statistiek:TNO, Leiden/ Heerlen, 2015.

10. Rijksinstituur voor Volksgezondheid en Milieu; Ministerie van Volksgezondheid, W.e.S. Ziekteverzuim en arbeidsongeschiktheid: Wat is de relatie met ziekten en aandoeningen? Nationaal Kompas Volksgezondheid 2014 [cited 2015 18-11-2015].

11. Chrousos, G.P., Stress and disorders of the stress system. Nat Rev Endocrinol, 2009. 5(7): p. 374-81.

2. H. Merskey, N.B., Part II: Pain Terms, A Current List with Definitions and Notes on Usage, in Classification of Chronic Pain. 1994, IASP Press: Seattle. p. 209-214.

13. Gatchel, R.J., et al., The biopsychosocial approach to chronic pain: scientific advances and future directions. Psychol Bull, 2007. 133(4): p. 581-624.

14. Williams, D.A., The importance of psychological assessment in chronic pain. Curr Opin Urol, 2013. 23(6): p. 554-9.

15. Turk, D.C. and A. Okifuji, Psychological factors in chronic pain: evolution and revolution. J Consult Clin Psychol, 2002. 70(3): p. 678-90

16. Stress and Pain, in Encyclopedia of Pain, G.F.S. Gebhart, R.F., Editor. 2013, Springer-Verlag: Berlin Heidelberg

17. Contrada, R.J. and A. Baum, eds. The Handbook of Stress Science: Biology, Psychology, and Health. 2010, Springer Publishing Company.

18. Habib, K.E., P.W. Gold, and G.P. Chrousos, Neuroendocrinology of stress. Endocrinol Metab Clin North Am, 2001 30(3): p. 695-728; vii-viii.

19. Cannon, W.B., Bodily changes in pain, hunger, fear and rage; an account of recent researches into the function of emotional excitement. 2d ed. 1929, New York, London:: D. Appleton and Company. xvi p., 1 1., 404 p. 
20. Ulrich-Lai, Y.M. and J.P. Herman, Neural regulation of endocrine and autonomic stress responses. Nat Rev Neurosci, 2009. 10(6): p. 397-409.

21. Charmandari, E., C. Tsigos, and G. Chrousos, Endocrinology of the stress response. Annu Rev Physiol, 2005. 67: p. 259-84.

22. Horrocks, P.M., et al., Patterns of ACTH and cortisol pulsatility over twenty-four hours in normal males and females. Clin Endocrinol (Oxf), 1990. 32(1): p. 127-34.

23. Vachon-Presseau, E., et al., The stress model of chronic pain: evidence from basal cortisol and hippocampal structure and function in humans. Brain, 2013. 136(Pt 3): p. 815-27.

24. Reinhardt, T., et al., Individual modulation of pain sensitivity under stress. Pain Med, 2013. 14(5): p. 676-85.

25. Generaal, E., et al., Biological stress systems, adverse life events and the onset of chronic multisite musculoskeletal pain a 6-year cohort study. Ann Rheum Dis, 2015.

26. Lampe, A., et al., Chronic pain syndromes and their relation to childhood abuse and stressful life events. Journal of psychosomatic research, 2003. 54(4): p. 361-7.

27. McBeth, J., et al., Moderation of psychosocial risk factors through dysfunction of the hypothalamic-pituitary-adrena stress axis in the onset of chronic widespread musculoskeletal pain: findings of a population-based prospective cohor study. Arthritis and rheumatism, 2007. 56(1): p. 360-71.

28. Ross, C., et al., Psychological Distress Mediates the Relationship Between Pain and Disability in Hand or Wrist Fractures. J Pain, 2015. 16(9): p. 836-43.

29. Wegener, S.T., et al., Psychological distress mediates the effect of pain on function. Pain, 2011. 152(6): p. 1349-57.

30. Klossika, I., et al., Emotional modulation of pain: a clinical perspective. Pain, 2006. 124(3): p. 264-8.

31. Moeller-Bertram, T., J. Keltner, and I.A. Strigo, Pain and post traumatic stress disorder - review of clinical and experimental evidence. Neuropharmacology, 2012. 62(2): p. 586-97.

32. Larsman, P., et al., Work related perceived stress and muscle activity during standardized computer work among female computer users. Work, 2009. 32(2): p. 189-99.

33. Lundberg, U., Stress responses in low-status jobs and their relationship to health risks: musculoskeletal disorders. Annals of the New York Academy of Sciences, 1999. 896: p. 162-72.

34. Thorn, S., et al., Trapezius muscle rest time during standardised computer work-a comparison of female computer users with and without self-reported neck/shoulder complaints. Journal of electromyography and kinesiology : official journal of the International Society of Electrophysiological Kinesiology, 2007. 17(4): p. 420-7.

35. Voerman, G.E., M.M. Vollenbroek-Hutten, and H.J. Hermens, Upper trapezius muscle activation patterns in neckshoulder pain patients and healthy controls. European journal of applied physiology, 2007. 102(1): p. 1-9.

36. Bansevicius, D., R.H. Westgaard, and T. Stiles, EMG activity and pain development in fibromyalgia patients exposed to mental stress of long duration. Scandinavian journal of rheumatology, 2001. 30(2): p. 92-8.

37. Lundberg, U., et al., Psychophysiological stress responses, muscle tension, and neck and shoulder pain among supermarket cashiers. Journal of occupational health psychology, 1999. 4(3): p. 245-55.

38. Rissen, D., et al., Surface EMG and psychophysiological stress reactions in women during repetitive work. European ournal of applied physiology, 2000. 83(2-3): p. 215-22.

39. Melzack, R., From the gate to the neuromatrix. Pain, 1999. Suppl 6: p. S121-6.
40. Koolhaas, J.M., et al., Stress revisited: a critical evaluation of the stress concept. Neurosci Biobehav Rev, 2011. 35(5): p. $1291-301$

41. Kyle, B.N. and D.W. McNeil, Autonomic arousal and experimentally induced pain: a critical review of the literature. Pain Res Manag, 2014. 19(3): p. 159-67.

42. Wall, P.D., S.B. McMahon, and M. Koltzenburg, Wall and Melzack's textbook of pain. 5th ed. ed. 2006, Philadelphia :: Elsevier/Churchill Livingstone

43. Giesler, G.J., Ascending Nociceptive Pathways, in Encyclopedia of Pain, G.F. Gebhart and R.F. Schmidt, Editors. 2013, Springer Berlin Heidelberg: Berlin, Heidelberg. p. 214-222.

44. Millan, M.J., Descending control of pain. Prog Neurobiol, 2002. 66(6): p. 355-474.

45. Wiech, K. M. Ploner, and I. Tracey, Neurocognitive aspects of pain perception. Trends Cogn Sci, 2008. 12(8): p. 306

46. Van Damme, S., et al., Keeping pain in mind: a motivational account of attention to pain. Neurosci Biobehav Rev, 2010. 34(2): p. 204-13

47. Quelhas Martins, A., D. McIntyre, and C. Ring, Aversive event unpredictability causes stress-induced hypoalgesia. Psychophysiology, 2015. 52(8): p. 1066-70.

48. Olango, W.M. and D.P. Finn, Neurobiology of stress-induced hyperalgesia. Curr Top Behav Neurosci, 2014. 20: p. 251

49. Jennings, E.M., et al., Stress-induced hyperalgesia. Prog Neurobiol, 2014. 121: p. 1-18.

50. Flor, H., et al., Pavlovian conditioning of opioid and nonopioid pain inhibitory mechanisms in humans. Eur J Pain, 2002. 6(5): p. 395-402

51. Sandkuhler, J., Models and mechanisms of hyperalgesia and allodynia. Physiol Rev, 2009. 89(2): p. 707-58.

52. Rhudy, J.L.M.M.W., The role of emotion in pain modulation. CURRENT OPINION IN PSYCHIATRY, 2001. 14(3): p. 241-

53. Tietjen, G.E., et al., Childhood maltreatment and migraine (part III). Association with comorbid pain conditions. Headache, 2010. 50(1): p. 42-51.

54. Goodwin, R.D., et al., Association between childhood physical abuse and gastrointestinal disorders and migraine in adulthood. Am J Public Health, 2003. 93(7): p. 1065-7.

55. Asmundson, G.J., et al., What is the role of fear and escape/avoidance in chronic pain? Models, structural analysis and future directions. Pain Manag, 2012. 2(3): p. 295-303.

56. Butler, R.K. and D.P. Finn, Stress-induced analgesia. Prog Neurobiol, 2009. 88(3): p. 184-202.

57. Geuze, E., et al., Altered pain processing in veterans with posttraumatic stress disorder. Arch Gen Psychiatry, 2007 64(1): p. $76-85$.

58. Ingram, R.E. and D.D. Luxton, Vulnerability-stress models. Development of psychopathology: A vulnerabilitystress perspective, 2005: p. 32-46

59. Lazarus, R.S. and S. Folkman, Stress, appraisal, and coping. 1984: Springer publishing company

60. Ursin, H. and H.R. Eriksen, The cognitive activation theory of stress. Psychoneuroendocrinology, 2004. 29(5): p. 567-92. 
61. Lupien, S.J., et al., Effects of stress throughout the lifespan on the brain, behaviour and cognition. Nat Rev Neurosci, 2009. 10(6): p. 434-45.

62. Korte, S.M. et al. The Darwinian concept of stress: benefits of allostasis and costs of allostatic load and the trade-offs in health and disease. Neurosci Biobehav Rev, 2005. 29(1): p. 3-38.

63. Logan, J.G. and D.J. Barksdale, Allostasis and allostatic load: expanding the discourse on stress and cardiovascular disease. Journal of Clinical Nursing, 2008. 17(7b): p. 201-208.

64. Carpenter, L.L., et al., Effect of childhood physical abuse on cortisol stress response. Psychopharmacology (Berl), 2011. 214(1): p. 367-75.

65. Heim, C., et al., Altered pituitary-adrenal axis responses to provocative challenge tests in adult survivors of childhood abuse. Am J Psychiatry, 2001. 158(4): p. 575-81.

66. Teicher, M.H., et al., The neurobiological consequences of early stress and childhood maltreatment. Neurosc Biobehav Rev, 2003. 27(1-2): p. 33-44.

67. Nicolson, N.A., et al., Childhood maltreatment and diurnal cortisol patterns in women with chronic pain. Psychosom Med, 2010. 72(5): p. 471-80.

68. Kendler, K.S., J.W. Kuhn, and C.A. Prescott, Childhood sexual abuse, stressful life events and risk for major depression in women. Psychol Med, 2004. 34(8): p. 1475-82.

69. Varese, F., et al., Childhood adversities increase the risk of psychosis: a meta-analysis of patient-control, prospectiveand cross-sectional cohort studies. Schizophr Bull, 2012. 38(4): p. 661-71.

70. Burns, J.W., The role of attentional strategies in moderating links between acute pain induction and subsequent psychological stress: evidence for symptom-specific reactivity among patients with chronic pain versus healthy nonpatients. Emotion (Washington, D C ), 2006. 6(2): p. 180-92.

71. Bartholomeusz, M.D., R. Callister, and D.M. Hodgson, Altered psychophysiological reactivity as a prognostic indicator of early childhood stress in chronic pain. Medical hypotheses, 2013. 80(2): p. 146-9.

72. Krantz, G., M. Forsman, and U. Lundberg, Consistency in physiological stress responses and electromyographic activity during induced stress exposure in women and men. Integr Physiol Behav Sci, 2004. 39(2): p. 105-18.

73. Hanvold, T.N., et al., The effect of work-related sustained trapezius muscle activity on the development of neck and shoulder pain among young adults. Scand J Work Environ Health, 2013. 39(4): p. 390-400.

74. Chou, R. and P. Shekelle, Will this patient develop persistent disabling low back pain? Jama, 2010. 303(13): p. 1295-302

75. Severeijns, R., et al., Pain catastrophizing predicts pain intensity, disability, and psychological distress independent of the level of physical impairment. Clin J Pain, 2001. 17(2): p. 165-72.

76. Dickerson, S.S. and M.E. Kemeny, Acute stressors and cortisol responses: a theoretical integration and synthesis of laboratory research. Psychol Bull, 2004. 130(3): p. 355-91.

77. Turk, D.C., A diathesis-stress model of chronic pain and disability following traumatic injury. Pain Research and Management, 2002. 7(1): p. 9-19.

78. Cacioppo, J.T., L.G. Tassinary, and G. Berntson, Handbook of Psychophysiology. 2007: Cambridge University Press.

79. Chang, B.S.S., D.L; Niedermeyer, E., Normal EEG and sleep: adults and elderly, in Electroencephalography: Basic Principles, clinical applications and related fields, F.L.d.S. Donald L. Schomer, Editor. 2011, Lippincott Williams \& Wilkins. p. 183-214.
80. Davidson, R.J., D.C. Jackson, and C.L. Larson, Human electroencephalography. Handbook of psychophysiology, 2000. 2: p. $27-52$.

81. Dustman, R.E., D.E. Shearer, and R.Y. Emmerson, Life-span changes in EEG spectral amplitude, amplitude variability and mean frequency. Clin Neurophysiol, 1999. 110(8): p. 1399-409.

82. Lewis, R.S., N.Y. Weekes, and T.H. Wang, The effect of a naturalistic stressor on frontal EEG asymmetry, stress, and health. Biol Psychol, 2007. 75(3): p. 239-47.

83. Master, S.L., et al., Neurobiological correlates of coping through emotional approach. Brain Behav Immun, 2009. 23(1): p. 27-35.

84. Goodman, R.N., et al., Stress, emotion regulation and cognitive performance: the predictive contributions of trait and state relative frontal EEG alpha asymmetry. Int J Psychophysiol, 2013. 87(2): p. 115-23.

85. Yu, X., et al., Relationship between scalp potential and autonomic nervous activity during a mental arithmetic task Auton Neurosci, 2009. 146(1-2): p. 81-6.

86. Ishitani, N., et al., Changes in electroencephalographic activities following pressure stimulation in humans. Psychiatry Clin Neurosci, 2005. 59(6): p. 644-51.

87. Cipresso, P., et al., EEG alpha asymmetry in virtual environments for the assessment of stress-related disorders. Stud Health Technol Inform, 2012. 173: p. 102-4

88. Coan, J.A. and J.J. Allen, Frontal EEG asymmetry as a moderator and mediator ofemotion. Biol Psychol, 2004. 67(1-2): p. 7-49.

89. Pizzagalli, D., Electroencephalography and high-density electrophysiological source localization, in Handbook of psychophysiology (3rd ed.), L.G.T. J. T. Caccioppo, \& G. Berntson, Editor. 2007, Cambridge University Press: Cambridge, UK. p. 56-84

90. Nitschke, J.B., Heller, W., Miller, G.A., Anxiety, stress, and cortical brain function. In Borod, J.C., The Neuropsychology of Emotion. 2000, New York: Oxford Universiy Press.

91. Oathes, D.J., et al., Worry, generalized anxiety disorder, and emotion: evidence from the EEG gamma band. Biol Psychol, 2008. 79(2): p. 165-70,

92. Peng, W., et al., Changes of spontaneous oscillatory activity to tonic heat pain. PLoS One, 2014. 9(3): p. e91052.

93. Gross, J., et al., Gamma oscillations in human primary somatosensory cortex reflect pain perception. PLoS Biol, 2007. 5(5): p. e133.

94. Chang, P.F., L. Arendt-Nielsen, and A.C. Chen, Dynamic changes and spatial correlation of EEG activities during cold pressor test in man. Brain Res Bull, 2002. 57(5): p. 667-75.

95. Vossen, C.J., et al., Does Habituation Differ in Chronic Low Back Pain Subjects Compared to Pain-Free Controls? A CrossSectional Pain Rating ERP Study Reanalyzed with the ERFIA Multilevel Method. Medicine (Baltimore), 2015. 94(19): p. $1-10$.

96. Vossen, C.J., et al., Introducing the event related fixed interval area (ERFIA) multilevel technique: a method to analyze the complete epoch of event-related potentials at single trial level. PLoS One, 2013. 8(11): p. e79905.

97. Krantz, G., M. Forsman, and U. Lundberg, Consistency in physiological stress responses and electromyographic activity during induced stress exposure in women and men. Integrative physiological and behavioral science : the official journal of the Pavlovian Society, 2004. 39(2): p. 105-18.

98. Tulen, J.H., et al., Characterization of stress reactions to the Stroop Color Word Test. Pharmacology, biochemistry, and behavior, 1989. 32(1): p. 9-15.

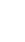


99. Lundberg, U., et al., Psychophysiological stress and EMG activity of the trapezius muscle. International journal of behavioral medicine, 1994. 1(4): p. 354-70.

100. Flodgren, G.M., et al., Changes in interstitial noradrenaline, trapezius muscle activity and oxygen saturation during low-load work and recovery. European journal of applied physiology, 2009. 107(1): p. 31-42.

101. Wahlstrom, J., et al., Influence of time pressure and verbal provocation on physiological and psychological reactions during work with a computer mouse. European journal of applied physiology, 2002. 87(3): p. 257-63.

102. Schleifer, L.M., et al., Mental stress and trapezius muscle activation under psychomotor challenge: a focus on EMG gaps during computer work. Psychophysiology, 2008. 45(3): p. 356-65.

103. Laursen, B., et al., Effect of mental and physical demands on muscular activity during the use of a computer mouse and a keyboard. Scandinavian journal of work, environment \& health, 2002. 28(4): p. 215-21.

104. Wahlstrom, J., et al., Perceived muscular tension, emotional stress, psychological demands and physical load during VDU work. International archives of occupational and environmental health, 2003. 76(8): p. 584-90.

105. Finsen, L., et al., Muscle activity and cardiovascular response during computer-mouse work with and without memory demands. Ergonomics, 2001. 44(14): p. 1312-29.

106. Svebak, S., R. Anjia, and S.I. Karstad, Task-induced electromyographic activation in fibromyalgia subjects and controls. Scandinavian journal of rheumatology, 1993. 22(3): p. 124-30.

107. Westgaard, R.H., et al., Trapezius activity of fibromyalgia patients is enhanced in stressful situations, but is similar to healthy controls in a quiet naturalistic setting: a case-control study. BMC Musculoskelet Disord, 2013. 14: p. 97.

108. Wijsman, J., et al., Trapezius Muscle EMG as Predictor of Mental Stress. Acm Transactions on Embedded Computing Systems, 2013. 12(4).

109. Willmann, M. and B. Bolmont, The trapezius muscle uniquely lacks adaptive process in response to a repeated moderate cognitive stressor. Neurosci Lett, 2012. 506(1): p. 166-9.

110. Flor, H., et al., Symptom-specific psychophysiological responses in chronic pain patients. Psychophysiology, 1992 29(4): p. 452-60.

111. Mark Burden, A.S.E. Lewis, and E. Willcox, The effect of manipulating root mean square window length and overlap on reliability, inter-individual variability, statistical significance and clinical relevance of electromyograms. Man The 2014. 19(6): p. 595-601.

112. Biondi, M. and A. Picardi, Psychological stress and neuroendocrine function in humans: the last two decades of research. Psychother Psychosom, 1999. 68(3): p. 114-50.

113. Ferrari, P.F., et al., Accumbal dopamine and serotonin in anticipation of the next aggressive episode in rats. Eur $J$ Neurosci, 2003. 17(2): p. 371-8.

114. Granovsky, Y., et al., Objective correlate of subjective pain perception by contact heat-evoked potentialls. J Pain, 2008. 9(1): p. 53-63.

15. Miltner, W., W. Larbig, and C. Braun, Habituation of subjective ratings and event related potentials to painful intracutaneous electrical stimulation of the skin. Journal of Psychophysiology, 1987. 1(3): p. 221-228,

116. Vossen, H., et al., More potential in statistical analyses of event-related potentials: a mixed regression approach. Int J Methods Psychiatr Res, 2011. 20(3): p. e56-68.

117. Myers, N.D., et al., Multilevel modeling in psychosomatic medicine research. Psychosomatic medicine, 2012. 74(9): p. 925-36. 


\section{Chapter 2}

Experimentally induced stress validated by EMG activity

Luijcks R1, Hermens $\mathrm{HJ}^{2}$, Bodar L1, Vossen $\mathrm{CJ}^{1,3}$, van Os J1,4, Lousberg R'

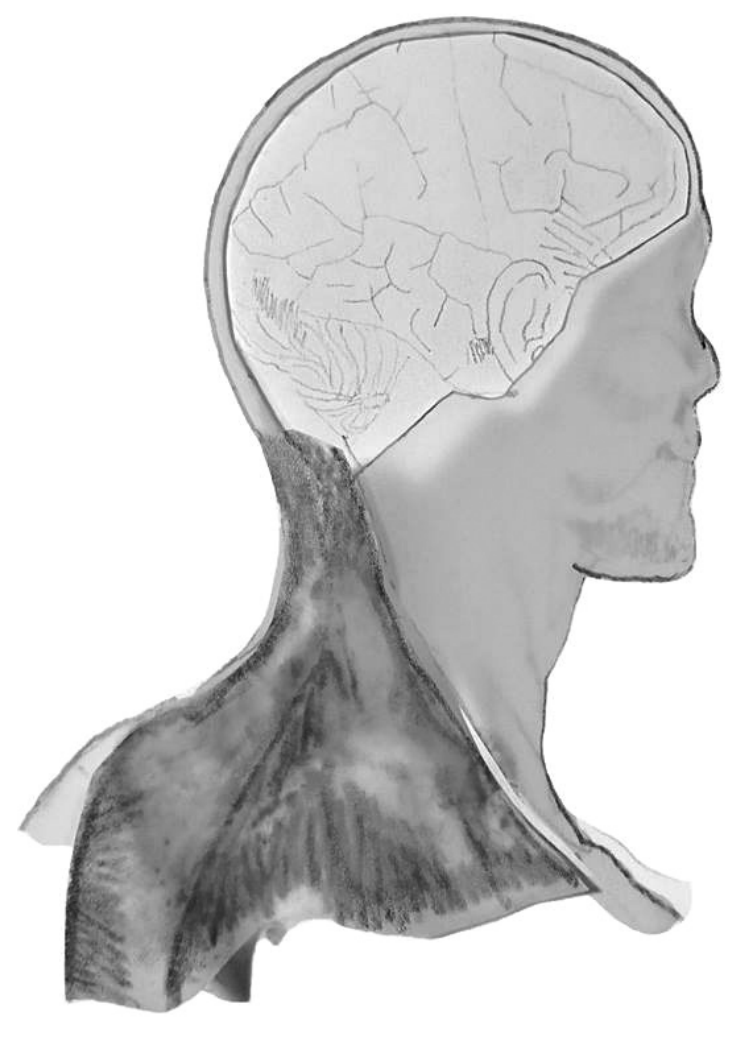

1 Department of Psychiatry \& Psychology, Maastricht University Medical Centre, The Netherlands

2 Roessingh Research and Development, Enschede, The Netherlands

列

4 King's College London, King's Health Partners, Department of Psychosis Studies, Institute of Psychiatry, London 


\section{ABSTRACT}

Experience of stress may lead to increased electromyography (EMG) activity in specific muscles compared to a non-stressful situation. The main aim of this study was to develop and validate a stress-EMG paradigm in which a single uncontrollable and unpredictable nociceptive stimulus was presented. EMG activity of the trapezius muscles was the response of interest. In addition to linear time effects, non-linear EMG time courses were also examined. Taking into account the hierarchical structure of the dataset, a multilevel random regression model was applied. The stress paradigm, executed in $\mathrm{N}=70$ subjects, consisted of a 3-minute baseline measurement, a 3-minute pre-stimulus stress period and a 2-minute post-stimulus phase. Subjects were unaware of the precise moment of stimulus delivery and its intensity level. EMG activity during the entire experiment was conform a priori expectations: the prestimulus phase showed a significantly higher mean EMG activity level compared to the other two phases, and an immediate EMG response to the stimulus was demonstrated. In addition, the analyses revealed significant non-linear EMG time courses in all three phases. Linear and quadratic EMG time courses were significantly modified by subjective anticipatory stress level, measured just before the start of the stress task. Linking subjective anticipatory stress to EMG stress reactivity revealed that subjects with a high anticipatory stress level responded with more EMG activity during the pre-stimulus stress phase, whereas subjects with a low stress level showed an inverse effect. Results suggest that the stress paradigm presented here is a valid test to quantify individual differences in stress susceptibility. Further studies with this paradigm are required to demonstrate its potential use in mechanistic clinical studies.

\section{INTRODUCTION}

Chronic stress is a risk factor for both somatic outcomes, such as hypertension[1-3], coronary heart disease [4], peptic ulcers [5-7] and asthma [8], and mental health outcomes including chronic 'benign' pain [9, 10], anxiety [11-13], PTSD [7, 14], depression [13, 15] and burn-out [15, 16]. In addition, work-related exposures such as repetitive work or mental stress during work, increase the risk for stress-related pain [17-23].

After Selye's theoretical and experimental pioneering work, more elaborate definitions of stress were proposed, including the concept of Levine and Ursin that makes a distinction between three elements: the input (stress stimuli), the processing systems (including the subjective experience of stress) and the output (stress responses) [24]. Stress, like pain, is best conceptualized as a theoretical construct, which is not directly measurable. A biopsychosocial approach to stress measurement may be useful, focusing on biological (somatic), psychological (behavioral) and social (cultural) dimensions of stress. To cover the three dimensions of stress, measurement should include both subjective measures (self-report questionnaires) and (psycho)physiological measures. The experience of stress is generally accompanied by an increased level of arousal and may lead to a number of physiological reactions, such as acceleration of the heart rate, pupil dilatation, increased galvanic skin response and increased finger pulse volume. Muscle activity measured with electromyography (EMG) also is sensitive to stress. The majority of experimental, clinical and field studies report an increase in EMG activity [25-34]. Increased EMG activity is prominent in the trapezius muscles, particularly if the stressor is personally relevant. Interestingly, however, some studies do not report such a relationship [35-37].

A well-known caveat in stress experiments is that the procedure of stress-induction is not under adequate experimental control. There are many possibilities to induce stress experimentally [38]. Examples include solving a mental arithmetic task, the Stroop word color test, examination stress, public speaking tests (e.g. the Trier Social Stress Test) or a combination of different stress tasks [39], but also paradigms in which subjects have to undergo an unpleasant physical stimulus, such as the cold pressure test [40] or electric shock test [35, 41-43]. It is unlikely that the above stress paradigms are unpredictable and/or uncontrollable to the same degree, which is relevant, given that one of the most crucial stress modifying factors is the degree to which a stimulus can be predicted and/or controlled [44-48]. In the stress paradigm of repeated (nociceptive) stimuli, the measurement of the direct stress response of each stimulus may be confounded by the phenomenon of habituation. In addition, experimental stress tasks may be confounded if accompanied by painful procedures not associated with the experiment itself, such as venipuncture. As a final critical point, it is important to note that there is an essential difference between a cognitive and a physical stressor. Most stress paradigms provide either a 
cognitive or a physical stressor. Studies providing both type of stressors at the same time are scarce (e.g. [25])

Led by these considerations, the goal of this study was to develop a novel and robust experimental stress paradigm according to the following principles. First, the stress paradigm should present a distinct cognitive and a distinct physical stressor. Second, it should include elements of uncontrollability and unpredictability, and third, it should be simple to administer, independent of illness status, educational level, motivation and other factors. To this end, a dual stress induction was introduced, consisting of (i) the announcement of imminent receipt of a single uncontrollable and unpredictable electric shock, thus inducing a cognitive anticipatory stress phase, followed by (ii) a nociceptive stimulus.

Within this experiment, three phases can be distinguished: (i) an anticipation, pre-stimulus phase, during which subjects are anticipating an electric shock in a relatively uncontrolled/ unpredictable situation; (ii) an immediate post-stimulus phase, showing the initial response to the nociceptive stimulus and (iii) a return to baseline phase, which can be described as the time required to achieve a comparable level of baseline physiological activity. Based on this paradigm, stress reactivity can be quantified and stored, reflecting individual differences in stress susceptibility.

The clear advantage of this paradigm is that the presentation of the physical stimulus has two functions: i) it makes the examination of a physical stressor possible and ii) it allows to demarcate the period of mental stress induction, since subjects were instructed that there would only be one physical stimulus.

A second goal of the study was to investigate in detail the muscle activation response during the stress condition. Experimental muscle response stress paradigms typically examine the linear time-related post-stimulus effects. This is logical, given the expectation that EMG activity increases while tension is building up in the anticipatory pre-stimulus phase. A linear time course suggests that the highest EMG activity would be reached just before receiving the stimulus, but considering the fact that the subjects receive an unpredictable stimulus, a more complex combination of linear and non-linear time effects may be more appropriate, for example in the form of a higher order polynomial function, which reaches a maximum. Modeling non-linear time effects is also relevant for the post-stimulus phase. Modeling an inverse time function (1/ time) is desirable, as this function portrays an initial sharp decrease or increase, followed by a plateau, which is applicable to the post-stimulus phase. EMG activity is typically measured by a computed average over a complete experimental condition. Thus, condition effects are tested by a comparison of the condition means. To this end, linear regression or ANOVA analyses are used. However, measuring EMG over time implicates a hierarchical structure of the data, in which consecutive time elements are nested within subjects. This hierarchical structure needs to be taken into account using multilevel random regression procedures [49].

We hypothesized that in comparison to baseline, EMG activity would increase during the prestimulus anticipation period, and would show a return to baseline in the post-stimulus period. In addition to the main hypothesis, we expected (i) non-linear EMG effects during pre- and post-stimulus stress periods, hypothesizing presence of both quadratic and inverse effects; (ii) asymmetric (left-right) EMG activity in the trapezius muscles, since we presented the electric stimulus unilaterally, on the left side; and (iii) given the multidimensional nature of stress, a positive association between subjective anticipatory stress on the one hand and EMG activity on the other. Further, in addition to a main effect, an interaction between the two different phases of the stress task and subjective anticipatory stress was expected. Since subjective stress is assumed to correlate positively with EMG activity, a larger increase in EMG activity within the pre-stimulus phase was expected in subjects with high levels of anticipatory stress, as compared to subjects with low levels of anticipatory stress.

\section{MATERIALS AND METHOD}

\section{Ethics Statement}

The study was conducted according to the principles of the Declaration of Helsinki and was approved by the medical ethics committee of the Academic Hospital Maastricht and Maastricht University (METC azM/UM, Maastricht). Before the start of the experiment, subjects provided written informed consent.

\section{Subjects}

Seventy right-handed subjects (44 females and 26 males) participated in the study. Their age ranged from 18 to 65 years. Exclusion criteria were structural use of antipsychotics, antiepileptics or anxiolytics during the past year or structural use of alcohol (>10 u/day). Subjects were asked to refrain from alcohol-containing consumptions the evening before and to refrain from caffeine-containing consumptions three hours prior to the experiment.

\section{Electro-shocker and stimuli}

An electro-shocker (type Shocko-100-AA-20, developed by Maastricht Instruments BV and approved for usage in experimental studies) was used to deliver electroshocks (see also [50]). Stimuli were electrical pulses of 10 milliseconds duration, administered intracutaneously on the top of the middle finger of the non-dominant left hand, as described by Bromm and Meier[51]. The sensation and pain threshold were determined by gradually increasing the intensity of 
the stimulus, starting at zero intensity. The first intensity that was consciously experienced was defined as the sensation threshold, the first intensity experienced as painful was defined as the pain threshold. This procedure was repeated three times in order to obtain a reliable estimate. The intensity of the electric stimulus applied during the experiment was computed for each subject individually. The intensity of the actually delivered stress stimulus during the experiment was calculated as follows:

Actually delivered stress stimulus $=$ pain threshold $+0.25 *$ (pain threshold - sensation threshold)

As shown in a previous experiment, this intensity level was experienced as painful by all subjects, albeit still acceptable [50].

\section{Procedure}

EMG-, and ECG-electrodes as well as the shock electrode were attached. EMG-electrodes were attached on the left and right trapezius muscle.

The baseline measurement was 3 minutes. After determination of the individual pain threshold subjects were instructed that they would receive a single electric shock sometime during a 5 -minute period. The experimenter pointed out that the precise moment of stimulus delivery and its intensity level would be determined by a personal computer. In addition, subjects were told that stimulus intensity might vary between the sensation threshold and a leve clearly above the pain threshold. Just before the start of the task, subjects had to answer the following question on a 10 point Likert scale: How much stress do you experience at this moment, awaiting the stimulus? Subjects were instructed to keep both hands on the table, palms down, and not to close their eyes during the whole measurement period. In fact, all subjects received the experimental stimulus at exactly $t=3$ minutes. The whole procedure was controlled by the software program "Presentation 0.71" (Neurobehavioral Systems).

\section{Psychophysiological recordings}

All recordings were conducted in an electrically and sound-shielded cubicle $\left(7.1 \mathrm{~m}^{2}\right)$. EMG activity was recorded from the left and right upper trapezius muscle. Electrodes were centered on a point $2 \mathrm{~cm}$ lateral to the midpoint between the acromion process and spinous process of the seventh cervical vertebra, using Ag/AgCl electrodes. A reference electrode was placed over the spinous process of the seventh cervical vertebra. Cardiac activity was recorded with a standard 3 lead ECG. All electrodes were fixed using 10-20 conductive paste. Brainvision BrainAmp Research Amplifier was used for all recordings. ECG and EMG were sampled with $1000 \mathrm{~Hz}$.

\section{Offline dataprocessing}

EMG data was filtered offline (low pass $0.5 \mathrm{~Hz}$, high pass $250 \mathrm{~Hz}$, $50 \mathrm{~Hz}$ notch filter) and segmented into epochs of 500ms. Raw data were visually inspected for artifacts and, if found, excluded from further analyses. EMG activity was corrected for ECG activity: the variance due to ECG activity was removed from the uncorrected EMG variable, using regression analysis. Next, for each $500 \mathrm{~ms}$ epoch, the root mean square value was calculated followed by a logarithmic transformation to preserve a normal distribution.

\section{Statistical analysis}

Given the hierarchical structure of the EMG dataset, consisting of epochs (level 1) that are clustered within individuals (level 2), multilevel random regression analyses were performed. EMG activity served as the dependent variable. Epoch number and condition, coded in two dummy variables contrasting baseline and post-stimulus versus pre-stimulus, served as independent variables in the basic model. Number of segment was included in order to investigate the linear effect over time. In addition to this linear time effect, a quadratic (epoch*epoch) and inverse effect (1/epoch) were added.

In order to test which covariance structure yielded the best fit for our dataset, various covariance structures were tested. Scaled Identity turned out to be significantly better than that of its competitors, namely compound symmetry (CS) and AR1. All models were tested with a random intercept. All statistical analyses were performed using SPSS 20.0. P-values below 0.05 were considered to be statistically significant.

\section{RESULTS}

Due to protocol violations (eyes closed, movements), 6 subjects were excluded from the analyses, leaving $n=64$ analyzable participants ( 40 females, 24 males).

Before analyzing the psychophysiological reactivity of the stress task, the amount of anticipatory stress experienced was analyzed. The mean score on the subjective stress item was 3.6 (SD = 2.61) with a score range from 0 to 10 . As expected, the mean score was significantly different from 0 ( $T=10.68, p<0.0001$ ), indicating that, on average, subjects experienced mild to moderate stress. Individual scores ranged from 0 to 10, indicative of large between-subject differences in experience of anticipatory stress.

\section{EMG activity during the three experimental phases}

Table 1 shows the results comparing the average left and right trapezius EMG activity for the baseline, pre-stimulus and post-stimulus periods. Mean EMG activity during the pre-stimulus 
period was significantly higher compared to baseline period (left trapezius muscle $T=32.13$, $\mathrm{p}<0.001$; right trapezius muscle $\mathrm{T}=31.33, \mathrm{p}<0.001$ ), whereas mean EMG activity during the poststimulus period was significantly lower compared to pre-stimulus period (left $\mathrm{T}=-6.70, \mathrm{p}<0.001$ right $T=-9.62, p<0.001$ ). An asymmetric (left-right) reaction in EMG activity was observed. The overall tension of the left trapezius muscle was higher than the tension of the right trapezius muscle during the whole experiment $\left(p<10^{-6}\right)$ (Table 1). The random intercepts of the models were significant (all $p<0.001$ ), indicating that EMG activity level at the start of each phase varied significantly between subjects.

Table 1. Mean EMG activity (in RMS) for baseline period, pre-stimulus period and post-stimulus period.

\begin{tabular}{lll}
\hline Period & $\begin{array}{l}\text { Left trapezius EMG activity } \\
\text { (mean and SD in log RMS) }\end{array}$ & $\begin{array}{l}\text { Right trapezius EMG activity } \\
\text { (mean and SD in log RMS) }\end{array}$ \\
\hline Baseline & $\mu=1.29[\sigma=0.29]$ & $\mu=1.24[\sigma=0.30]$ \\
Pre-stimulus & $\mu=1.38[\sigma=0.30]$ & $\mu=1.34[\sigma=0.31]$ \\
Post-stimulus & $\mu=1.36[\sigma=0.29]$ & $\mu=1.30[\sigma=0.32]$ \\
\hline
\end{tabular}

The linear time* condition interaction effect of EMG activity was investigated. As hypothesized EMG activity showed a significant increase during the pre-stimulus period compared to the baseline period, for both the left $(T=2.31, p=0.021)$ and the right $(T=2.46, p=0.014)$ trapezius muscle. In comparison with the pre-stimulus period, a very significant contrasting (decreasing EMG activity) linear time*condition interaction was observed in the post-stimulus phase, again both left ( $T=-15.84, p=<0.0001)$ and right $(T=-12.38, p=<0.0001)$.

Non-linear EMG time effects were modeled post-hoc, by including a quadratic and inverse component as predictor variables in the multilevel model. The results of this model are shown in figure 1.

An inverse effect was observed at all periods, consisting of a rapid initial decline of EMG values immediately after the start of each period, followed by a plateau phase. The inverse and parabolic effects were significantly different between the pre- and post-stimulus period, for both the left $(T=5.53, p<0.0001)$ and right $(T=4.20, p<0.0001)$ trapezius muscle. With regard to the parabolic component, there was a statistically significant pre-post difference, only for the right trapezius muscle $(T=-2.46, p=0.014)$. This quadratic difference indicates that the parabolic decrease in the EMG activity of the right trapezius muscle in the post-stimulus period was larger than the parabolic decrease in the pre-stimulus period.

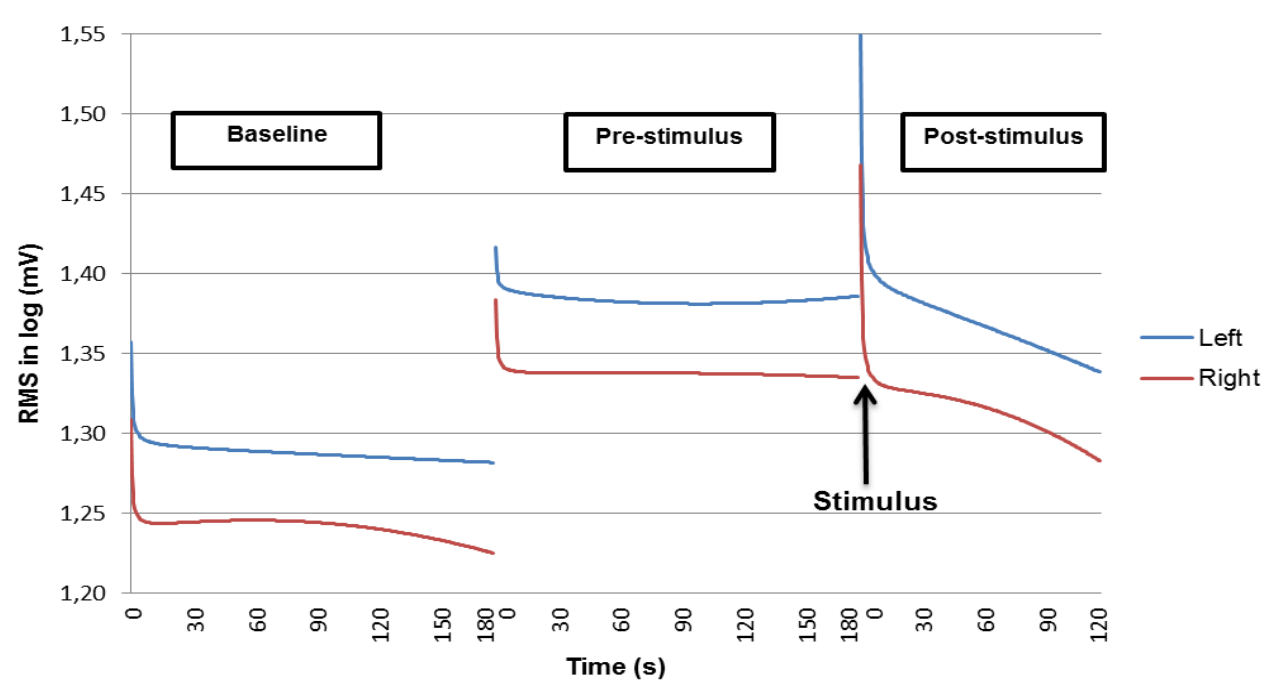

Figure 1. Modeled non-linear EMG activity in trapezius muscle for baseline, pre- and poststimulus period.

Figure 1 shows that a complete return of EMG activity to baseline level (i.e. mean EMG activity in the baseline period) did not take place in the post-stimulus phase

\section{Response to the nociceptive stimulus}

Figure 2 shows the short-term effect of the experimental stimulus on EMG activity for a time window ranging from 10 seconds pre-stimulus to 15 seconds post-stimulus. The immediate EMG effect of the nociceptive stimulus consisted of a sharp increase, both left and right, followed by a pronounced decrease. The time required to reach an EMG activity level equal to or lower than the level just before the stimulus is about 15 seconds (Fig. 2).

\section{Subjective anticipatory stress and EMG activity}

Including the non-linear time components (quadratic and inverse) in the model, it was tested whether the mean EMG activity level in pre- and post-stimulus phase was independently associated with the subjective anticipatory stress level. Contrary to the hypothesis, this appeared to be the case in neither the pre-stimulus nor the post-stimulus period, for both the left and the right trapezius muscle (all $p>0.70$ ).

We examined whether both linear and non-linear time courses within the pre- and poststimulus period interacted with subjective anticipatory stress. In order to visualize the interaction effects as contrasting as possible, only the predicted EMG time course of the 


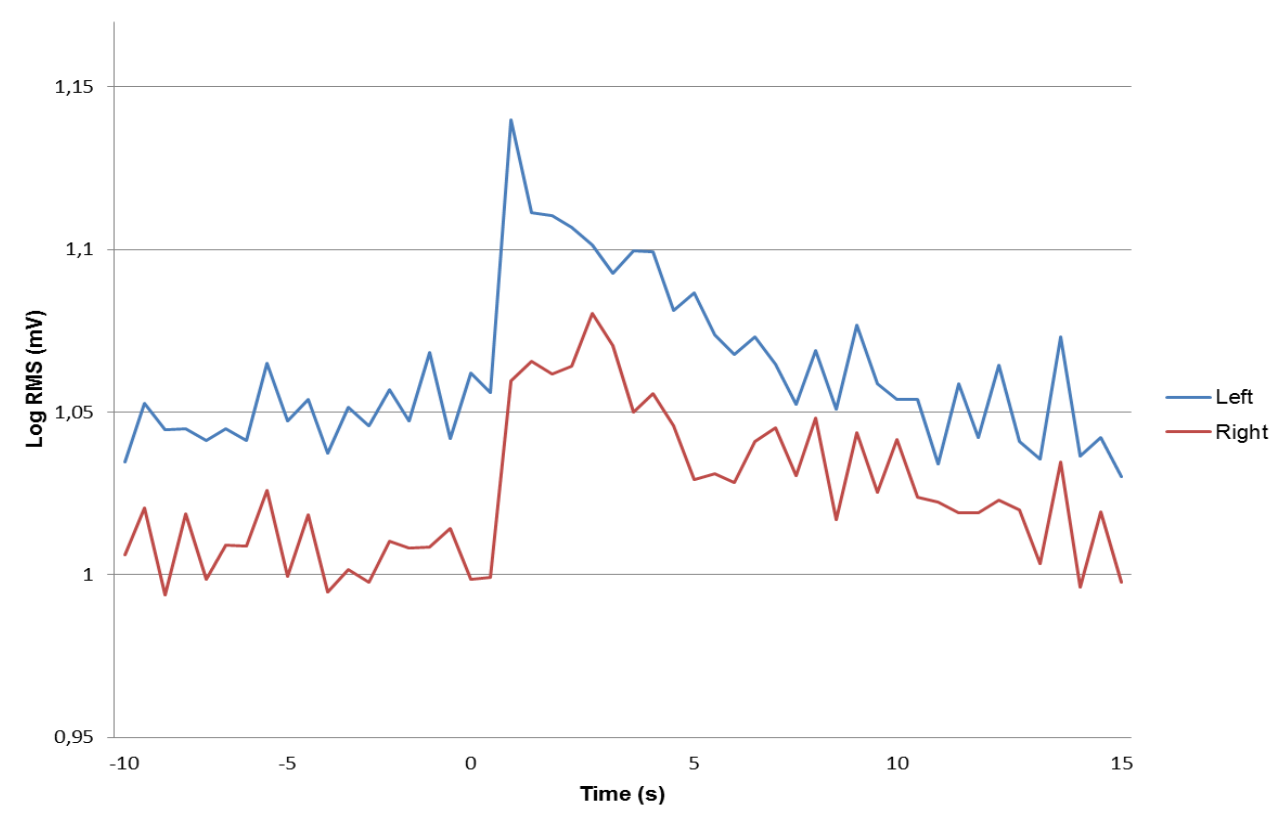

Figure 2. Direct stimulus response, showing a time interval of 10 seconds pre-stimulus to 15 seconds post-stimulus.

highest anticipatory stress score (10) and the lowest anticipatory stress score (0) was plotted. This revealed strong differences in the (non-linear) time course of the stress task between the extremes (Fig.3 and 4). Testing the hypothesized greater level of EMG activity in subjects experiencing more stress in the pre-stimulus phase revealed that both left and right trapezius muscle showed this effect: subjects with the highest stress level (score 10), showed a quadratic initial increase of EMG activity during the pre-stimulus period, while subjects with a zero stress level tended to display an initial decrease during the pre-stimulus period. The multilevel models, including the anticipatory stress scale as a continuous variable, showed highly significant linear and parabolic effects (all $p<0.001$ ). No interaction effects for the inverse time component were found.

The results of a final post hoc analysis, concerning the post-stimulus period, were less conclusive. Whereas in the left trapezius muscle significant linear and parabolic interaction effects were apparent, these effects were not evident in the right trapezius muscle.

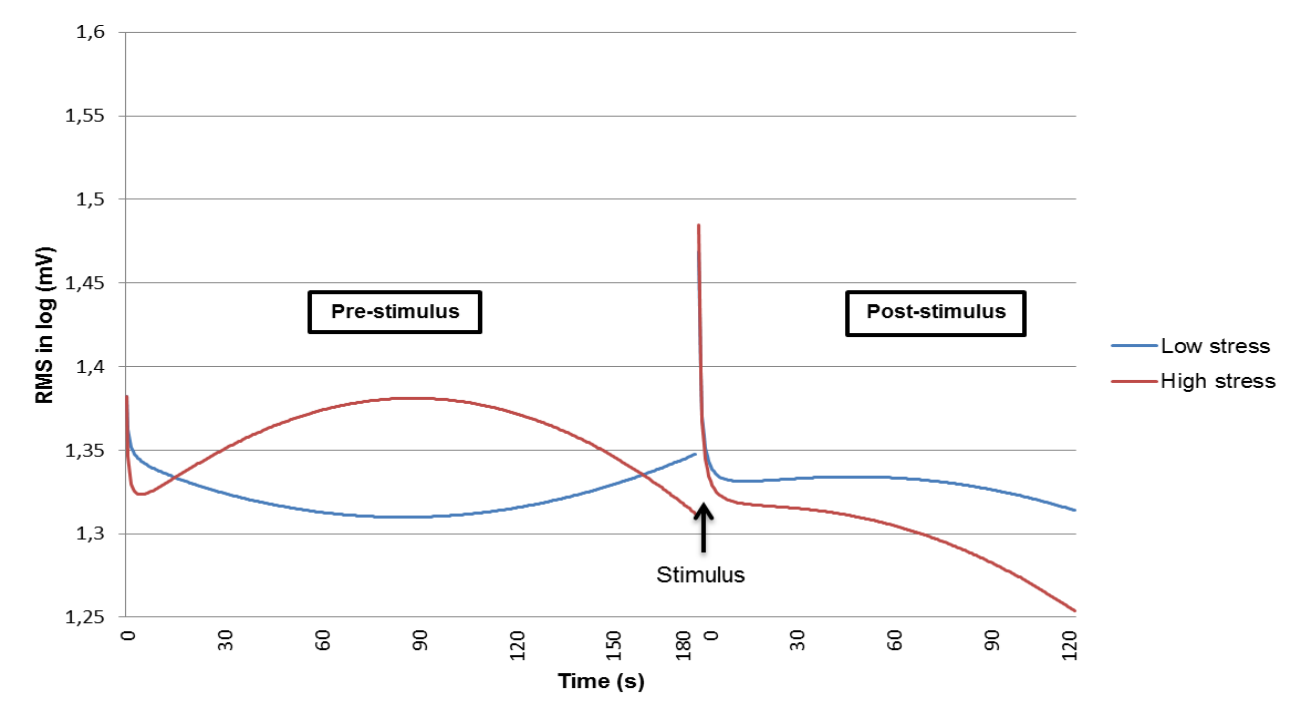

Figure 3. Modeled non-linear time course during the stress task interacted with subjective anticipatory stress. Low and high stress levels are contrasted, for the left trapezius muscle.

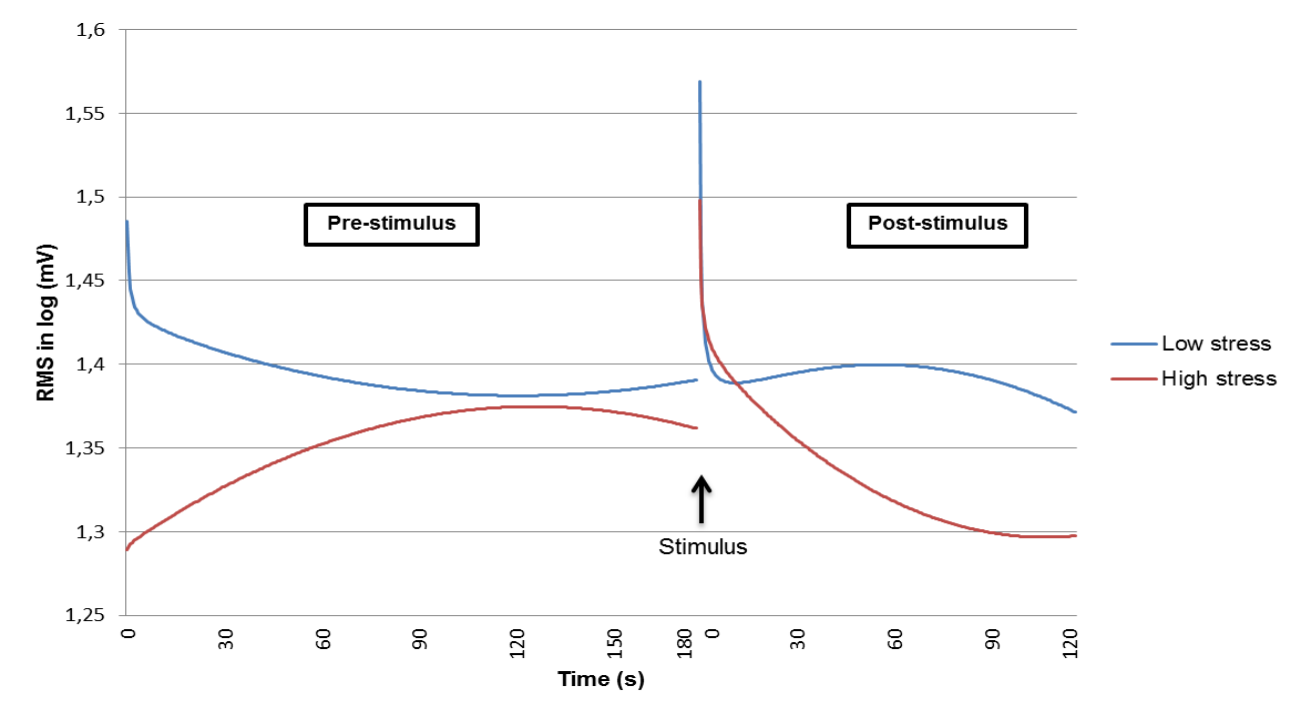

Figure 4. Modeled non-linear time course during the stress task interacted with subjective anticipatory stress. Low and high stress levels are contrasted, for the right trapezius muscle. 


\section{DISCUSSION AND CONCLUSION}

\section{General EMG effects}

The key finding of this study was that, in agreement with the a priori hypotheses, significant differences in mean EMG activity were observed (in both left and right trapezius), between the three experimental phases of a novel experimental stress paradigm including a cognitive and a physical stressor. In addition, a clear response to the experimental nociceptive stimulus was demonstrated. Mean EMG activity level was higher in the pre-stimulus period compared to both the baseline and the post-stimulus phase. Further, during all three periods, a linear time course in EMG was present. During the pre-stimulus phase there was a linear increase in EMG activity compared to baseline, followed by a decreasing linear course during the post-stimulus phase.

Non-linear EMG time courses were observed. The pronounced inverse effect, characterized by a rapid decline just after the start of each of the three phases, may be explained as follows: with respect to the baseline period and the pre-stimulus phase, the EMG recording started immediately after instructions were given by the experimenter. This 'event' may have caused an increase in EMG activity, which rapidly disappeared after the start of the measurement. The inverse effect at the start of the post-stimulus phase is attributed to the stimulus reactivity. In addition to these inverse effects, parabolic effects were also modeled. In contrast to the inverse effects, no parabolic time component in EMG activity was significant.

In all models, a significant random intercept was observed, indicating that there was random (intercept) variability in EMG starting level between subjects. This so-called intercept variability in 'habitual EMG level' is a well-known phenomenon in (clinical) practice. It can be concluded that multilevel analyses are required when investigating EMG activity in this type of stress experiment.

Anticipatory stress level, measured just before the start of the stress task, was mild to moderate on average. Given subjective experience of stress, with parallel evidence of changes in EMG activity, suggests that the stress task was validated. In addition, EMG activity during the three phases was demonstrated in the expected direction given the dual nature (the cognitive and nociceptive stress elements) of the stress task. The relative increase (compared to the baseline period) during the pre-stimulus phase can be regarded as the cognitive stress response. The immediate, sharp increase after the experimental stimulus can be regarded as the nociceptive stress response.

\section{Influence of subjective stress on EMG activity}

There was no association between the level of subjective anticipatory stress and the mean EMG activity level, not even during the pre-stimulus phase. Apparently, the intuitive assumption that subjective stress is expressed in a general rise in EMG activity does not apply. Interpersonal differences in this relationship, related to third variables including personality factors, may create heterogeneity. Some examples of these potentially relevant personality characteristics are maladaptive coping strategies such as catastrophizing [52], state anxiety level [53] and specific personality traits, such as neuroticism [13]. In this respect, it becomes clear that in a proper analysis of EMG variability, a multidimensional perspective is necessary.

When modeling the quadratic and inverse time effects, it was found that the EMG non-linear time course during the pre-stimulus phase was moderated by anticipatory stress level. For both left and right trapezius muscle in the pre-stimulus phase, EMG activity developed exactly in the direction which was predicted: subjects with high anticipatory stress showed an initial increase, whereas subjects with low anticipatory-stress showed an opposite EMG time course. The observation that the top of the fitted parabola was reached within the pre-stimulus phase may be associated with the unpredictability, i.e. the uncertainty about the exact point of time the stimulus would be received.

Although different EMG time courses were observed during the post-stimulus phase, there was no consistency between the left and right trapezius muscle. This left-right inconsistency in the post-stimulus phase may be explained by the fact that the stimulus was applied to only one (left) hand, leading to an asymmetry in EMG activity. More experimental research to unravel this issue is required.

Some methodological issues are apparent. First, since return to baseline has been suggested as a useful stress parameter $[10,54]$, it would have been interesting to carry out analyses on this measure. The results made clear (figure 1), however, that the post-stimulus period was too short to reach a complete return to the EMG activity level of the baseline phase. Therefore, in a future experiment, the length of the post-stimulus period should be prolonged. A second point is that one could argue that the response to the physical stimulus is a superimposed reaction on an existing mental stress state, and thus might be biased. This is true, but the ideal situation, in which an experimentally controlled physical stressor is presented without the knowledge of the subject, might be considered as unethical. Further, stress experiments using a series of physical stimuli, implicitly contain a mental stress element. In other words, it seems impossible to set up a physical-stress paradigm without also inducing a mental stressor. Another critical note pertains the generalizability of the results of the stress task: the current paradigm may be considered as a rigid stress task, performed in a laboratory setting. This 
undoubtedly has its limitations to the generalization to daily life stress situations. On the other hand, the exact timing of the physical stressor and the identical environment for all subjects facilitates the examination of individual variability of stress reactivity and aides in linking stress mechanisms to subjective experienced stress levels.

In sum, a new stress paradigm has been developed which contains a dual stress induction presenting both a mental stressor as well as a physical stressor. Additionally, the stress task is straight-forward and easy to administer in a broad population, independent of illness status, educational level, motivation and other factors. The results of the analyses provide evidence for the validity of the stress task. The present paradigm may be used in future studies on fundamental stress mechanisms. Dependent on the research question, other stress-related (psycho)physiological parameters can be incorporated. This stress task may also be used to assess the relationship between experimentally induced psychophysiological stress reactivity and a subsequent health outcomes. As such, it may possibly be useful as a diagnostic tool in clinical practice, and contribute to high-risk preventive paradigms.

\section{ACKNOWLEDGMENTS}

We want to thank Ron Mengelers for providing technical support.

\section{REFERENCES}

1. Jones, A., et al., Adiposity is associated with blunted cardiovascular, neuroendocrine and cognitive responses to acute mental stress. PloS one, 2012. 7(6): p. e39143.

2. Esler, M., et al., Chronic mental stress is a cause of essential hypertension: presence of biological markers of stress. Clinical and experimental pharmacology \& physiology, 2008. 35(4): p. 498-502.

3. Sparrenberger, F., et al., Does psychosocial stress cause hypertension[quest] A systematic review of observational studies. J Hum Hypertens, 2008. 23(1): p. 12-19.

4. Richardson, S., et al., Meta-Analysis of Perceived Stress and Its Association With Incident Coronary Heart Disease. American Journal of Cardiology, 2012. 110(12): p. 1711-1716

5. Levenstein, S., G.A. Kaplan, and M. Smith, Sociodemographic characteristics, life stressors, and peptic ulcer. A prospective study. Journal of clinical gastroenterology, 1995. 21(3): p. 185-92.

6. Levenstein, S., G.A. Kaplan, and M.W. Smith, Psychological predictors of peptic ulcer incidence in the Alameda County Study. Journal of clinical gastroenterology, 1997. 24(3): p. 140-6.

7. Handwerger, K., Differential patterns of HPA activity and reactivity in adult posttraumatic stress disorder and major depressive disorder. Harvard review of psychiatry, 2009. 17(3): p. 184-205.

8. Wright, R.J., M. Rodriguez, and S. Cohen, Review of psychosocial stress and asthma: an integrated biopsychosocial approach. Thorax, 1998. 53(12): p. 1066-74

9. Flor, H., et al., Symptom-specific psychophysiological responses in chronic pain patients. Psychophysiology, 1992 29(4): p. 452-60.

10. Flor, H. and D.C. Turk, Psychophysiology of chronic pain: do chronic pain patients exhibit symptom-specific psychophysiological responses? Psychological bulletin, 1989. 105(2): p. 215-59.

11. Strohle, A. and F. Holsboer, Stress responsive neurohormones in depression and anxiety. Pharmacopsychiatry, 2003 36 Suppl 3: p. S207-14

12. Abelson, J.L. and G.C. Curtis, Hypothalamic-pituitary-adrenal axis activity in panic disorder. 24-hour secretion of corticotropin and cortisol. Archives of general psychiatry, 1996. 53(4): p. 323-31.

13. Uliaszek, A.A., et al., The role of neuroticism and extraversion in the stress-anxiety and stress-depression relationships. Anxiety, Stress \& Coping, 2009. 23(4): p. 363-381.

14. de Kloet, C.S., et al., Enhanced cortisol suppression in response to dexamethasone administration in traumatized veterans with and without posttraumatic stress disorder. Psychoneuroendocrinology, 2007. 32(3): p. 215-26.

15. Chrousos, G.P. and P.W. Gold, The concepts of stress and stress system disorders. Overview of physical and behavioral homeostasis. JAMA : the journal of the American Medical Association, 1992. 267(9): p. 1244-52.

16. Tyssen, R., et al., The impact of job stress and working conditions on mental health problems among junior house officers. A nationwide Norwegian prospective cohort study. Medical education, 2000. 34(5): p. 374-84.

17. Larsman, P., et al., Work related perceived stress and muscle activity during standardized computer work among female computer users. Work, 2009. 32(2): p. 189-99.

18. Lundberg, U., Stress responses in low-status jobs and their relationship to health risks: musculoskeletal disorders Annals of the New York Academy of Sciences, 1999. 896: p. 162-72.

19. Thorn, S., et al., Trapezius muscle rest time during standardised computer work-a comparison of female computer users with and without self-reported neck/shoulder complaints. Journal of electromyography and kinesiology : official journal of the International Society of Electrophysiological Kinesiology, 2007. 17(4): p. 420-7. 
20. Voerman, G.E., M.M. Vollenbroek-Hutten, and H.J. Hermens, Upper trapezius muscle activation patterns in neckshoulder pain patients and healthy controls. European journal of applied physiology, 2007. 102(1): p. 1-9.

21. Bansevicius, D., R.H. Westgaard, and T. Stiles, EMG activity and pain development in fibromyalgia patients exposed to mental stress of long duration. Scandinavian journal of rheumatology, 2001. 30(2): p. 92-8.

22. Lundberg, U., et al., Psychophysiological stress responses, muscle tension, and neck and shoulder pain among supermarket cashiers. Journal of occupational health psychology, 1999. 4(3): p. 245-55.

23. Rissen, D., et al., Surface EMG and psychophysiological stress reactions in women during repetitive work. European journal of applied physiology, 2000. 83(2-3): p. 215-22.

24. Levine, S., Ursin, H., What is stress?, in Stress, Neurobiology and Neuroendocrinology, M.R. Brown, Rivier, C., Koob, G. (Eds.), Editor 1991, Marcel Decker: New York. p. 3-21.

25. Krantz, G., M. Forsman, and U. Lundberg, Consistency in physiological stress responses and electromyographic activity during induced stress exposure in women and men. Integrative physiological and behavioral science : the official journal of the Pavlovian Society, 2004. 39(2): p. 105-18.

26. Tulen, J.H., et al., Characterization of stress reactions to the Stroop Color Word Test. Pharmacology, biochemistry, and behavior, 1989. 32(1): p. 9-15.

27. Lundberg, U., et al., Psychophysiological stress and EMG activity of the trapezius muscle. International journal of behavioral medicine, 1994. 1(4): p. 354-70.

28. Flodgren, G.M., et al., Changes in interstitial noradrenaline, trapezius muscle activity and oxygen saturation during low-load work and recovery. European journal of applied physiology, 2009. 107(1): p. 31-42

29. Wahlstrom, J., et al., Influence of time pressure and verbal provocation on physiological and psychological reactions during work with a computer mouse. European journal of applied physiology, 2002. 87(3): p. 257-63.

30. Schleifer, L.M., et al., Mental stress and trapezius muscle activation under psychomotor challenge: a focus on EMG gaps during computer work. Psychophysiology, 2008. 45(3): p. 356-65,

31. Laursen, B., et al., Effect of mental and physical demands on muscular activity during the use of a computer mouse and a keyboard. Scandinavian journal of work, environment \& health, 2002. 28(4): p. 215-21.

32. Wahlstrom, J., et al., Perceived muscular tension, emotional stress, psychological demands and physical load during VDU work. International archives of occupational and environmental health, 2003. 76(8): p. 584-90.

33. Finsen, L., et al., Muscle activity and cardiovascular response during computer-mouse work with and without memory demands. Ergonomics, 2001. 44(14): p. 1312-29.

34. Svebak, S., R. Anjia, and S.I. Karstad, Task-induced electromyographic activation in fibromyalgia subjects and controls. Scandinavian journal of rheumatology, 1993. 22(3): p. 124-30.

35. Noteboom, J.T. K.R. Barnholt, and R.M. Enoka, Activation of the arousal response and impairment of performance increase with anxiety and stressor intensity. Journal of applied physiology, 2001. 91(5): p. 2093-2101.

36. Blangsted, A.K., et al., The effect of physical and psychosocial loads on the trapezius muscle activity during computer keying tasks and rest periods. European journal of applied physiology, 2004. 91(2-3): p. 253-8.

37. Bansevicius, D., R.H. Westgaard, and C. Jensen, Mental stress of long duration: EMG activity, perceived tension fatique, and pain development in pain-free subjects. Headache, 1997. 37(8): p. 499-510.

38. Biondi, M. and A. Picardi, Psychological stress and neuroendocrine function in humans: the last two decades of research. Psychotherapy and psychosomatics, 1999. 68(3): p. 114-50.
39. Reinhardt, T., et al. Salivary cortisol heart rate, electrodermal activity and subjective stress responses to the Mannheim Multicomponent Stress Test (MMST). Psychiatry research, 2012. 198(1): p. 106-111.

40. Schwabe, L., L. Haddad, and H. Schachinger, HPA axis activation by a socially evaluated cold-pressor test. Psychoneuroendocrinology, 2008. 33(6): p. 890-5.

41. Breznitz, S., et al., Experimental induction and termination of acute psychological stress in human volunteers: effects on immunological, neuroendocrine, cardiovascular, and psychological parameters. Brain, behavior, and immunity, 1998. 12(1): p. 34-52.

42. Weisse, C.S., et al., Differential effects of controllable and uncontrollable acute stress on lymphocyte proliferation and leukocyte percentages in humans. Brain, behavior, and immunity, 1990. 4(4): p. 339-51.

43. Arntz, A. and R. Lousberg, The effects of underestimated pain and their relationship to habituation. Behaviour research and therapy, 1990. 28(1): p. 15-28.

44. Oka, S., et al. Predictability of painful stimulation modulates subjective and physiological responses. The journal of pain : official journal of the American Pain Society, 2010. 11(3): p. 239-46.

45. Weiss, J.M... Effects of coping responses on stress. Journal of comparative and physiological psychology, 1968. 65(2): p. $251-60$

46. Maier, S. and L. Watkins, Stressor Controllability, Anxiety, and Serotonin. Cognitive Therapy and Research, 1998 22(6): p. 595-613.

47. Koolhaas, J.M., et al., Stress revisited: a critical evaluation of the stress concept. Neuroscience and biobehaviora reviews, 2011. 35(5): p. 1291-301.

48. Arntz, A., M. van Eck, and P. de Jong, Avoidance of pain of unpredictable intensity. Behaviour research and therapy, 1991. 29(2): p. 197-201.

49. Myers, N.D., et al., Multilevel modeling in psychosomatic medicine research. Psychosomatic medicine, 2012. 74(9): p. 925-36.

50. Vossen, H., et al., More potential in statistical analyses of event-related potentials: a mixed regression approach. international journal of methods in psychiatric research, 2011. 20(3): p. e56-68.

51. Bromm, B. and W. Meier, The intracutaneous stimulus: a new pain model for algesimetric studies. Methods and findings in experimental and clinical pharmacology, 1984. 6(7): p. 405-10.

52. Karsdorp, P.A., et al., Pain catastrophizing, threat, and the informational value of mood: task persistence during a painful finger pressing task. Pain, 2012. 153(7): p. 1410-7.

53. Endler, N.S. and N.L. Kocovski, State and trait anxiety revisited. Journal of anxiety disorders, 2001. 15(3): p. 231-45.

54. Traue, H.C., et al., Nonverbal expressiveness and EMG activity in tension headache sufferers and controls. Journal of psychosomatic research, 1985. 29(4): p. 375-81. 


\section{Chapter 3}

Impact of early life adversity on EMG stress reactivity of the trapezius

muscle

R Luijcks'; CJ Vossen 1,2; S Roggeveen'; J van Os ${ }^{1,3} ;$ HJ Hermens ${ }^{4,5}$ and R Lousberg'

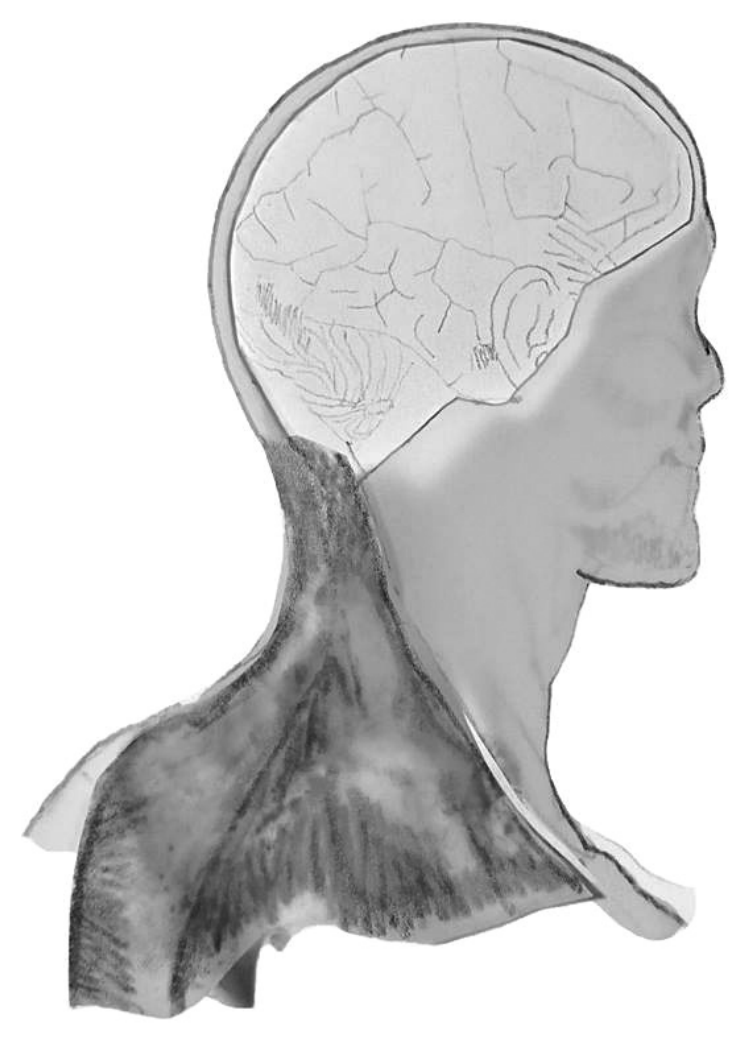

1 Department of Psychiatry \& Psychology, Maastricht University Medical Centre, Maastricht, The Netherlands Department of Anesthesiology and Pain $M$

Netherlands

King's College London, King's Health Partners, Department of Psychosis Studies, Institute of Psychiatry, London, United Kingdom

作

5 Telemedicine Group, Faculty of Electrical Engineering, Mathematics and Computer Science, University of Twente 


\section{ABSTRACT}

Human and animal research indicates that exposure to early life adversity increases stress sensitivity later in life. While behavioral markers of adversity-induced stress-sensitivity have been suggested, physiological markers remain to be elucidated. It is known that trapezius muscle activity increases during stressful situations. The present study examined to what degree early life adverse events experienced during early childhood (0-11 years) and adolescence (12-17 years) moderate experimentally induced electromyographic (EMG) stress activity of the trapezius muscles, in an experimental setting. In a general population sample $(n=115)$, an anticipatory stress effect was generated by presenting a single unpredictable and uncontrollable electrical painful stimulus at $t=3$ minutes. Subjects were unaware of the precise moment of stimulus delivery and its intensity level. Linear and non-linear time courses in EMG activity were modelled using multilevel analysis. The study protocol included two experimental sessions ( $t=0$ and $t=6$ months) allowing for examination of reliability.

Results show that EMG stress reactivity during the stress paradigm was consistently stronger in people with higher levels of early life adverse events; early childhood adversity had a stronger moderating effect than adolescent adversity. The impact of early life adversity on EMG stress reactivity may represent a reliable facet that can be used in both clinical and non-clinical studies.

\section{INTRODUCTION}

Exposure to early life adversity (also called adverse childhood experiences, both terms are used interchangeably) is a risk factor for developing affective and psychotic disorders later in life [1-4] and is similarly associated with functional somatic syndromes including pain [5, 6]. Exposure to adverse childhood experiences (ACEs) increases stress sensitivity later in life [7-9]. A stress sensitization model has been hypothesized, which postulates that childhood adversity increases vulnerability to mental disorders following adult stressful life events [79]. In addition, several studies have demonstrated that ACEs have a lasting impact on adult physiology, including neurobiological processes, immunological processes and autonomic endocrine and metabolic systems [10-16]. Hamer et al. suggest that (psycho)physiological reactivity to mental stress can be viewed as a robust characteristic, indicating that stressinduced responses do not habituate over (a short period of) time [17].

Stressors experienced during childhood can be highly variable, including neglect or sexual abuse, but also parental separation or divorce. The literature indicates that significant early life adversity is not rare in western societies [18-20]. Dong et al. demonstrated that different ACEs were significantly interrelated, and that the influence of adverse childhood experiences on behavior, physical health and mental disorders is cumulative $[9,21]$. Age at the time of traumatization also may influence the effects in later life, earlier exposure being associated with more harmful effects [22, 23].

While behavioral markers of trauma-induced stress sensitivity have been suggested [7-9] physiological markers of trauma-induced stress sensitivity remain to be elucidated. EMG stress reactivity may be an interesting candidate physiological marker in relation to early life adversity. It is known that muscle activity increases during stressful situations [24-28]. In particular, trapezius muscle activity can be influenced by stress [24, 27-30], making the trapezius muscle a possible candidate for examining the impact of early life adversity on a physiological stressrelated outcome.

In the present study, we examined the relationship between ACEs and muscle activity in a recently developed stress experiment [26]. In this paradigm, an anticipatory stress effect is generated by inducing both a distinct cognitive stressor and a physical painful stimulus. The anticipatory stress effect is mediated by an increase in EMG activity of the trapezius muscle during the pre-stimulus phase [26].

The first objective was to investigate the association between ACEs, experienced during early childhood and adolescence, and stress-related trapezius muscle activity. The second objective was to examine the reliability of any influence of ACEs on EMG stress reactivity. A stress 
experiment was applied, in which a single unpredictable and uncontrollable electrical painful stimulus was presented. Given the relatively high rate of adverse childhood experiences in the general population [31, 32], a general population sample was included. In order to investigate the reliability of the influence of ACEs on EMG stress reactivity, the study protocol included two experimental sessions for each participant. The first took place at the moment of inclusion, the second six months later. The protocol was identical across the two experimental sessions.

Measuring EMG over time implicates a hierarchical structure of the data, in which consecutive time elements are nested within subjects. This hierarchical structure needs to be taken into account. Consequently, multilevel random regression was used [33]. In addition, it has been shown that EMG activity measured during a stress experiment comprises non-linear time effects [26], which can be modeled by multilevel regression. In order to approach the natura effects expected to be present in anticipatory muscle activity, it was decided to include a linear, exponential and quadratic time effect in the analyses. A linear increase in muscle activity was expected, since the anticipatory stress phase is associated with an increase in tension, resulting in heightened muscle activity. Additionally, non-linear time effects were also expected. A quadratic time effect could be expected: a parabola opening upwards or downwards, representing either an initial relaxation and a consecutive increase of tension, or an initial building up of muscle activity, followed by a relative relaxation afterwards. In addition to the quadratic time effect, an exponential effect may be present, representing a relatively stable muscle activity during the first part of the anticipation phase, followed by a growth in tension in which the growth is proportional to the current level of tension.

It was hypothesized that exposure to early life adversity, particularly those occurring during early childhood, would be associated with increased trapezius muscle activity during the anticipatory phase of the stress task.

\section{METHODS}

\section{Ethics Statement}

The study was conducted according to the principles of the Declaration of Helsinki and approved by the medical ethics committee of the Maastricht University Medical Centre and Maastricht University (NL40284.068.12/METC 12-3-015). Subjects provided written informed consent before the start of the experiment.

\section{Subjects}

The experiment is part of a larger study. Participants consisted of a general population sample residing in the city of Maastricht, the Netherlands, and had responded to flyers. Between june 2012 and april 2015, one-hundred-fifteen right-handed subjects (74 females and 4 males), aged 18 to 65 years, participated in the study. Exclusion criteria were use of alcoholic beverages in excess of 10 units per day and structural use of antidepressants, anti-epileptics, antipsychotics or anxiolytics during the past year. Subjects were asked to not use alcoholcontaining consumptions the evening before and caffeine-containing consumptions three hours prior to the experiment. Recompense for time spent was 50 euros.

\section{Electro-shocker and stimuli}

An electro-shocker (type Shocko-100-AA-20, developed by Maastricht Instruments BV and approved for use in experimental studies) was used to deliver electrical stimuli (see also Vossen et al. [34]). Stimuli were electrical pulses of 10 milliseconds duration, administered intracutaneously on the top of the middle finger of the non-dominant left hand, as described by Bromm and Meier [35]. The sensation and pain threshold were determined for all subjects, starting at zero intensity, followed by a gradual increase in stimulus intensity. The first intensity that was consciously experienced was defined as the sensation threshold. The pain threshold was defined as the first intensity experienced as painful. The procedure of determining these personal thresholds was repeated three times in order to attain reliable estimates. The intensity of the electrical stimulus applied during the experiment was calculated for each subject separately. This intensity level was experienced as painful by all subjects, but still acceptable [34]. The intensity of the stress stimulus that was delivered during the experiment was calculated as follows:

\section{Stimulus $=$ pain threshold $+0.25^{*}($ pain threshold - sensation threshold $)$}

\section{Procedure}

EMG- and ECG-electrodes, as well as the shock electrode, were attached. EMG-electrodes were placed on the skin above the left and right trapezius muscle. After determination of the individual pain threshold, subjects were instructed that they would receive a single electrical shock at some time during a 5-minute period. The experimenter pointed out that the intensity level of the electrical stimulus and the precise time of delivery would be determined by a personal computer. Additionally, subjects were told that stimulus intensity might vary between the sensation threshold and a level clearly above the pain threshold. Subjects were instructed to keep both hands on the table, palms down, and to not close their eyes during the entire measurement period. All subjects received the experimental stimulus at exactly $t=3$ minutes. The procedure was controlled by the software program "Presentation 0.71" (Neurobehavioral Systems). 
The study protocol included two experimental sessions for each participant. The first took place at the moment of inclusion, the second six months later. The protocol was identical across the two experimental sessions.

\section{Psychophysiological recordings}

All recordings were conducted in an electrically and sound-shielded cubicle (7.1 m2). EMG activity was recorded from the left and right upper trapezius muscle. Electrodes were centered on a point $2 \mathrm{~cm}$ lateral to the midpoint between the acromion process and spinous process of the seventh cervical vertebra [36], using Ag/AgCl electrodes. A reference electrode was placed over the spinous process of the seventh cervical vertebra. Cardiac activity was recorded with a standard 3-lead ECG. All electrodes were fixed using conductive paste. Brainvision BrainAmp Research Amplifier was used for all recordings. ECG and EMG were sampled with $1000 \mathrm{~Hz}$.

\section{Psychological measurements}

Early life adversity was assessed at the moment of inclusion, with a questionnaire developed within the context of the FP7 EU-GEl project (European network of national schizophrenia networks studying Gene-Environment Interactions) [37]. The Childhood Experiences of Care and Abuse questionnaire comprises 15 questions on adverse childhood events, like the divorce of parents, the presence of financial problems, the occurrence of sexual abuse, etcetera.

The questionnaire covers two age categories: the first category includes ACEs between 0 and 11 years (early childhood), the second category ACEs between 12 and 17 year (adolescence). Cronbach's alpha coefficient was 0.68 and 0.64 for the two respective age categories. The maximum score in each age category was 15 , the maximum score for the entire questionnaire was 30. The sum of scored events for both categories together ranged from 0 to 13 (mean 3.1 SD 3.0). For the exposure category of $0-11$ years, the sum of scored events ranged from 0 to 8 (mean 1.6, SD 1.8), whereas for the exposure category of $12-17$ years, the sum of scored events ranged from 0 to 7 (mean 1.4, SD 1.6).

\section{Offline data processing}

EMG data was filtered offline (low pass $0.5 \mathrm{~Hz}$, high pass $250 \mathrm{~Hz}, 50 \mathrm{~Hz}$ notch filter) and segmented into epochs of $512 \mathrm{~ms}$. Raw data were visually inspected for artifacts which, if encountered, were excluded from the analyses. The EMG activity of the trapezius muscle was corrected for cardiac activity: the variance due to ECG activity was removed from the uncorrected EMG variable, using regression analysis. Next, since the number of epochs was restricted due to hardware memory limitations, three consecutive epochs were merged, resulting in a total of 117 analyzable consecutive epochs. For each $1536 \mathrm{~ms}$ epoch, the root mean square value was calculated followed by a logarithmic transformation, in order to preserve a normal distribution

\section{Statistical analysis}

Given the hierarchical structure of the EMG dataset, consisting of epochs (level 1), nested within experimental sessions (level 2), that are clustered within individuals (level 3), multilevel random regression analyses were performed (see appendix for model). Although no conclusive evidence is provided in the literature on differences between left and right muscle activity during experimental stress, some studies do report differences [38-41]. Thus, EMG activity of the left and right trapezius muscle (LTM and RTM respectively) served as the dependent variable in all models. In order to obtain normality, the dependent variables were log-transformed. Epoch number was included in order to investigate the linear effect over time. In addition to the linear time effect, a quadratic (epoch ${ }^{2}$ ) and exponential (e $\left.e^{\text {epoch }}\right)$ time effect were added. Associations of interest were interactions between the time variables and the linear ACE-score. Additionally, the interactions between the time variables and the two age subcategories of ACE exposure (early childhood and adolescence) were examined. Analyses were adjusted for age and sex.

Additional analyses were carried out in order to examine the reliability of the influence of ACEs on the time course of EMG activity. Experimental session was included as predictor, both as main effect as well as interaction term. Three third-order interaction terms were modeled: epoch*ACE-score*session, epoch ${ }^{2 *}$ ACE-score* ${ }^{*}$ session and e epoch*ACE-score*session.

In order to examine which covariance structure yielded the best fit for the dataset, various covariance structures were tested. The -2 log likelihood of different models was calculated in order to determine which statistical model would fit best. An autoregression (AR1) structure showed the best fit (lowest -2 log likelihood). As the dataset has a multilevel structure consisting of consecutive epochs, each epoch is correlated with the previous epoch, which makes an autoregression model suitable. The AR1 structure was therefore used for all statistical analyses.

All models were tested with a random intercept and random slope for the linear effect of time. All statistical analyses were performed using SPSS 22.0. Two-sided P-values $<.05$ were considered statistically significant.

\section{RESULTS}

Ten subjects were excluded from the analyses due to protocol violations (movements, not following instructions), leaving $n=105$ analyzable participants (67 females, 38 males). Ages ranged from 18 to 65 years, with a mean age of 38.6 years (SD 17.1). 


\section{Influence of ACEs on EMG stress reactivity}

ACE-score was included as a continuous variable in the models. Table 1 shows the $\mathrm{T}$ - and p-values of all time effects included in the model, i.e. linear, quadratic and exponential time. When examining this table, it can be seen that for both left and right trapezius muscles, the effects are comparable. For the linear time*ACE interaction, a positive T-value is present for both LTM and RTM ( $T=3.676$ and $T=3.070$, respectively), indicating a relatively higher increase in EMG activity over time for higher ACE-scores: the higher the score, the more increase in muscle activity. Second, the quadratic time*ACE interaction shows us a negative T-value ( $T=-$ 4.879 for the LTM, T=-2.879 for the RTM), representing a parabola with a downward opening Based on the data depicted in the table, it can be concluded that for higher scores on the ACE questionnaire, a higher maximum in muscle activity will be reached (i.e. the top of the parabola). The exact course of the EMG activity should be calculated for each score separately. Finally, the exponential time*ACE interaction has a positive T-value for both trapezius muscles ( $T=3.847$ and $T=2.185$ ). These results mean that, from a certain point during the anticipatory stress phase, muscle activity builds up prior to the imminent painful stimulus. The exponentia building of tension is more apparent for higher ACE-scores. All ACE*time interactions, both left and right, were significant. The effects on the right trapezius muscle were somewhat smaller than on the left trapezius muscle.

Table 1. Interactions between total ACE-score on EMG time effects

\begin{tabular}{lllll} 
& \multicolumn{2}{r}{ Left Trapezius Muscle (LTM) } & \multicolumn{1}{c}{ Right Trapezius Muscle (RTM) } \\
\hline Time*ACE & $\mathrm{T}=3.475^{* *}$ & $\mathrm{p}=0.001^{* *}$ & $\mathrm{~T}=3.015^{* *}$ & $\mathrm{p}=0.003^{* *}$ \\
Time $^{2 *}$ ACE & $\mathrm{T}=-4.548^{* *}$ & $\mathrm{p}<0.001^{* *}$ & $\mathrm{~T}=-2.789^{* *}$ & $\mathrm{p}=0.005^{* *}$ \\
$\mathrm{e}^{\mathrm{t} \text { ime* ACE }}$ & $\mathrm{T}=3.668^{* *}$ & $\mathrm{p}<0.001^{* *}$ & $\mathrm{~T}=2.107^{*}$ & $\mathrm{p}=0.035^{*}$ \\
\hline
\end{tabular}

Values shown are T-values, indexing time*linear ACE-score interaction effects. Three-level regression models were used including level of EMG response, experimental session and subject EMG activity of the RTM and the LTM served as the dependent vaibles. Linear, quadalc and exponential time as well as their interctions with ACE-score as the depente served as the fixed effects of main interest. Results are marked with one asterisk $\left(^{*}\right)$ if $p<.05$ and two $\left(^{* *}\right)$ if $p<.01$. Corresponding fitted time course of EMG activity is illustrated in Fig. 1 .

For illustrative purposes, it was decided to depict the non-linear EMG time course (predicted by the multilevel model) during the anticipatory stress phase for two relatively extreme ACEscores: a score of 0 (representing no ACEs) and a score of 13 (maximum observed score in this sample). In order to obtain a predicted EMG time course, the scores of 0 and 13 were inserted in the regression model respectively (see appendix).

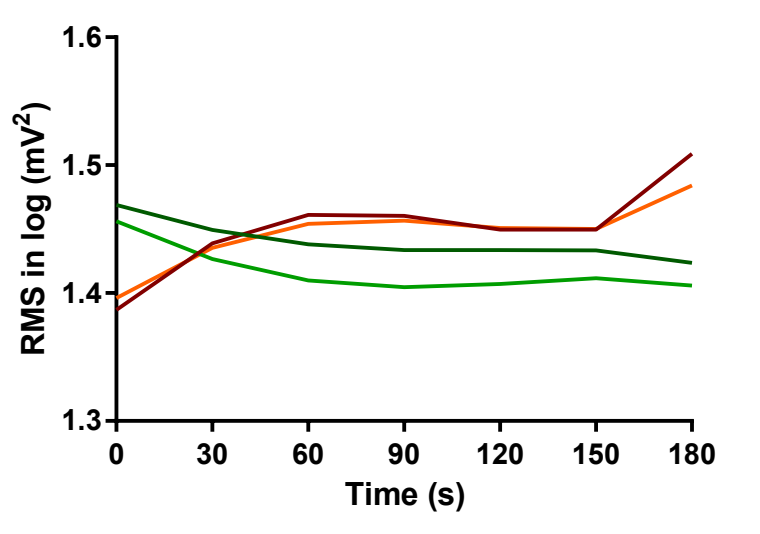

- Low ACE - LTM

- High ACE - LTM

- LOW ACE - RTM

- High ACE - RTM

Fig. 1. Fitted time course of EMG activity during the anticipatory stress period, interacted with ACE-score. LTM indicates the left trapezius muscle, RTM indicates the right trapezius muscle. For illustrative purposes, the EMG time course was calculated for a high ACE-score (13 events, i.e. maximum score in this sample) and a low ACE-score (0 events).

\section{Influence of early life adversity on EMG stress reactivity across different} ACE-exposure age categories

We investigated whether different EMG time interaction effects for the two age subcategories of the ACE-questionnaire, early childhood and adolescence, could be demonstrated. The number of adverse childhood experiences in the age category of 0 to 11 years is associated with increased muscle activity during the anticipatory phase for both the LTM and the RTM. The results show similar T- and $p$-values as described above: a positive linear time*ACE interaction, a negative quadratic time*ACE interaction and a positive exponential time*ACE interaction. For the ACE-exposure age category of 12-17 years, however, a less prominent interaction effect between EMG time course and the number of ACEs experienced was observed. A significant interaction between EMG time course and ACE-score was only apparent for the left trapezius muscle.

Similarly as for the overall effects, for both ACE-exposure age categories, the extreme scores were inserted into the computed multilevel models. For illustrative purposes, figure 2 shows the predicted time courses for the two ACE-score extremes observed in this sample: a minimum score of 0 events, a maximum of 8 events.

In all models, age was associated with left and right trapezius muscle activity ( $p=077$ and $p \leq .005$ respectively). An association between sex and muscle activity could not be demonstrated (both $\mathrm{p} \geq .836$ ). Finally, all models showed a significant random intercept and a random slope for linear time (all $p<.001$ ) 
Table 2. The effect of ACE-score on EMG time effects, per age category.

\begin{tabular}{ccccccccc}
\hline & \multicolumn{3}{c}{ Left Trapezius Muscle (LTM) } & \multicolumn{2}{c}{ Right Trapezius Muscle (RTM) } \\
\hline \multicolumn{3}{c}{ Age 0-11 years } & Age 12-17 years & Age 0-1 1 years & Age 12-17 years \\
Time*ACE & $T=4.482^{* * *}$ & $p<0.001^{* *}$ & $T=1.542$ & $p=0.124$ & $T=3.576^{* *}$ & $p<0.001 * *$ & $T=1.684$ & $p=0.093$ \\
Time $^{2 * A C E}$ & $T=-5.542^{* *}$ & $p<0.001^{* *}$ & $T=-2.378^{*}$ & $p=0.017^{*}$ & $T=-3.442^{* *}$ & $p=0.001 * *$ & $T=-1.407$ & $p=0.160$ \\
$\mathbf{e}^{\text {time*ACE }}$ & $T=4.224^{* *}$ & $p<0.001^{* *}$ & $T=2.195^{*}$ & $p=0.028^{*}$ & $T=2.694^{* *}$ & $p=0.007^{* *}$ & $T=0.955$ & $p=0.339$
\end{tabular}

Values shown are T-values and $p$-values, indexing time*ACE-score interactions. Three-level regression models were used including level of EMG response, experimental session and subject. EMG activity of the RTM and the LTM served as the dependent variables. Linear, quadratic and exponential time as well as their interactions with ACE-score served as the fixed effects of main interest. Results are marked with one asterisk (*) if $p<.05$ and two $\left.{ }^{* *}\right)$ if $p<.01$. For the interaction between time and ACE-score during childhood (0-11 years), corresponding fitted time course of EMC activity is illustrated in Fig. 2.

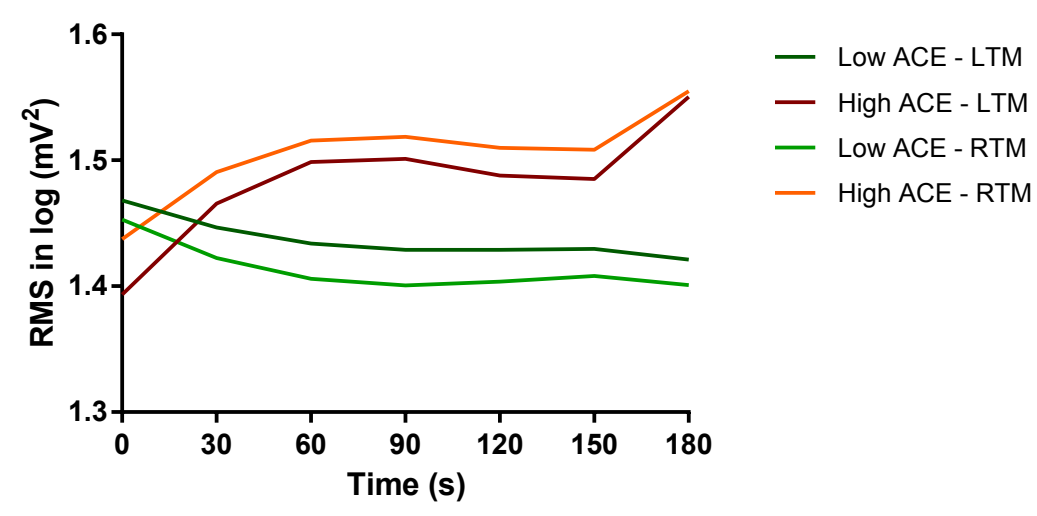

Fig. 2. Fitted time course of EMG activity during the anticipatory stress period, in interaction with ACE-score in exposure age-category 0 to 11 years. LTM indicates the left trapezius muscle, RTM indicates the right trapezius muscle. The time course of EMG activity for a high ACE-score (8 events, i.e. maximum score in this sample) and a low ACE-score (0 events) was calculated, in order to demonstrate the contrasting process.

\section{Reliability of the impact of ACEs on EMG stress reactivity}

In order to examine the reliability of the influence of ACEs on time course of EMG activity, additional analyses were carried out, including experimental session as predictor variable in the model. Three third-order interaction terms were modeled: epoch*ACE-score*session,

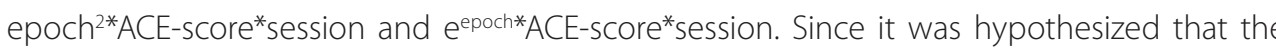

influence of childhood adversity on EMG stress reactivity would be stable over time, no difference in ACE-effect between both experimental sessions was expected. Table 3 shows the $T$ - and $p$-values of these third-order interaction effects. No significant results were apparent (all $p \geq .175$ ).

Table 3. Interactions between total ACE-score, time and experimental session.

\begin{tabular}{|c|c|c|c|c|}
\hline \multirow[b]{2}{*}{ Time*ACE*Experimental session } & \multicolumn{2}{|c|}{ Left Trapezius Muscle (LTM) } & \multicolumn{2}{|c|}{ Right Trapezius Muscle (RTM) } \\
\hline & $\mathrm{T}=-1.228$ & $p=0.220$ & $T=-0.470$ & $p=0.638$ \\
\hline Time $\mathrm{e}^{2 *} \mathrm{ACE}{ }^{*}$ Experimental session & $T=0.612$ & $p=0.541$ & $\mathrm{~T}=0.572$ & $p=0.567$ \\
\hline $\mathrm{e}^{\text {time*} A C E^{*} \text { Experimental session }}$ & $T=0.235$ & $p=0.814$ & $T=-0.457$ & $p=0.648$ \\
\hline
\end{tabular}

Values shown are T-values and $\mathrm{p}$-values indexing time ${ }^{*}$ ACE-score ${ }^{*}$ session interactions. Three-level regression models were used including level of EMG response, experimental session and subject. EMG activity of the RTM and the LTM served as the dependent variables. Linear, quadratic and exponential time as well as their interactions with ACE-score and experimental session served as the fixed effects of main interest. No interaction effects were apparent, indicating invariable session effects.

\section{DISCUSSION}

We investigated the relationship between early life adversity and EMG stress reactivity in adulthood. Although several adult stress-related outcomes are moderated by adverse childhood experiences [10-12], no previous studies reported on the influence of ACEs on EMG reactivity as a psychophysiological stress response. An association between ACEs and altered EMG reactivity was found, particularly for ACEs in early childhood. The association was reliable over time. These findings were conform the a priori hypotheses.

A higher ACE-score was accompanied by increased muscle activity in both trapezius muscles during the anticipatory stress phase. Early life adversities can lead to higher levels of stress sensitivity in adulthood, possibly caused by dysregulation or hyperactivity of the HPA-axis. This could result in long-lasting effects on psychophysiological activity [10-12, 17]. The exponentia time course of the EMG reactivity effect, marked by a sharp increase at the end of the anticipatory phase for high ACE-scores, is instructive. This effect may be related to uncertainty about the exact timing of the painful stimulus. It indicates that subjects who have experienced more early life adversity tend to become tenser as the stressor (i.e. the unpredictably painfu stimulus) is approaching. An interrelationship between adverse childhood experiences, inadequate or immature coping and stress-related symptoms has been described in previous research [42-45]. Inadequate coping, associated with early life adversity, may lead to increased stress sensitivity/vulnerability for mental disorders later in life. 
Early childhood ACEs (occurred between 0 and 11 years) were associated with increased EMG stress reactivity, for both trapezius muscles. For ACEs that occurred during adolescence (12-17 years) this was less prominent and apparent only for the LTM. These results are in line with previous research $[22,23]$, showing more enhanced effects on adult mental health outcomes associated with exposure to adversity at a younger age.

A left-right difference in interaction with EMG reactivity was observed throughout the analyses, the left trapezius muscle showing greater reactivity than the right. This difference may be explained by the fact that the stimulus was applied to the left hand. Second, since it was assumed that the variables age and sex could impact stress reactivity, these variables were included as covariates. The significant main effect of age demonstrated a negative association with the degree of muscle activity. No influence of sex emerged.

Finally, invariability of EMG stress responsivity across measurement occasion was demonstrated. No inter-visit ( $\mathrm{t}=0$ and $\mathrm{t}=6$ months) difference was observed, suggesting a reliable influence of ACEs on psychophysiological activity. The invariability confirms the robustness of the demonstrated anticipatory stress effects. Furthermore, it increases the plausibility that altered stress reactivity plays a role in the development of stress-related health problems.

Natural stress responses may not likely be solely linear. Both exponential and quadratic time effects were demonstrated, which affirms this assumption. One of the strengths of multilevel regression techniques is that random time effects can be modelled. In the present study, a highly significant random intercept as well as random linear time effect were demonstrated. The significant random intercept, indicating different EMG levels between persons, was expected since EMG is influenced by many other factors like posture and body morphology (see Wijsman et al. [28]). The significant random time effect has to be interpreted as the existence of different slopes of linear time courses across subjects. Stated otherwise, every subject reacts differently to the stressor, a phenomenon for which the main effects are corrected. Traditional ANOVA techniques do not permit estimation of random effects.

\section{Limitations of the study}

An experimental painful stimulus was used, which can be viewed as a minor variant of an extensive collection of daily stressors. In daily life, stressors rapidly alternate. The question is to what degree the results of the present experimental study can be extended to daily life situations.

\section{CONCLUSION}

This is, to our knowledge, the first study to investigate the influence of early life adversity on
EMG stress reactivity of the trapezius muscles. In sum, results showed robust alterations in EMG stress reactivity for subjects with a history of adverse childhood experiences. Examining clinical populations may be productive in further unravelling the mechanisms underlying the stress response and its relation to mental health problems.

\section{ACKNOWLEDGEMENTS}

We would like to thank Lonneke Bodar and Marga Schnitzeler for their supportive role in this study and Jacco Ronner and Ron Mengelers for technical support. All statistical methods were developed with adequate in house statistical support. 


\section{REFERENCES}

Kendler KS, Kuhn JW, Prescott CA. Childhood sexual abuse, stressful life events and risk for major depression in women. Psychological medicine. 2004;34(8):1475-82. PubMed PMID: 15724878.

2. Heim C, Newport DJ, Mletzko T, Miller AH, Nemeroff CB. The link between childhood trauma and depression: insights from HPA axis studies in humans. Psychoneuroendocrinology. 2008;33(6):693-710. doi: 10.1016/1. psyneuen.2008.03.008. PubMed PMID: 18602762

3. Varese F, Smeets F, Drukker M, Lieverse R, Lataster T, Viechtbauer W, et al. Childhood adversities increase the risk of psychosis: a meta-analysis of patient-control, prospective- and cross-sectional cohort studies. Schizophr Bul. 2012;38(4):661-71. doi: 10.1093/schbul/sbs050. PubMed PMID: 22461484; PubMed Central PMCID: PMC3406538.

4. McLaughlin KA, Greif Green J, Gruber MJ, Sampson NA, Zaslavsky AM, Kessler RC. Childhood adversities and firs onset of psychiatric disorders in a national sample of US adolescents. Arch Gen Psychiatry. 2012;69(11):1151-60. doi: 10.1001/archgenpsychiatry.2011.2277. PubMed PMID: 23117636; PubMed Central PMCID: PMC3490224.

5. Afari N, Ahumada SM, Wright LJ, Mostouf S, Golnari G, Reis V, et al. Psychological trauma and functional somatic syndromes: a systematic review and meta-analysis. Psychosomatic medicine. 2014;76(1):2-11. doi: 10.1097/ PSY0000000000000010 PubMed PMID: 24336429; PubMed CentratPMCID. PMC3894419.

6. Lampe A, Doering S, Rumpold G, Solder E, Krismer M, Kantner-Rumplmair W, et al. Chronic pain syndromes and their relation to childhood abuse and stressful life events. Journal of psychosomatic research. 2003;54(4):361-7. PubMed PMID: 12670615

7. Lardinois M, Lataster T, Mengelers R, Van Os J, Myin-Germeys I. Childhood trauma and increased stress sensitivity in psychosis. Acta psychiatrica Scandinavica. 2011;123(1):28-35. doi: 10.1111/j.1600-0447.2010.01594.x. PubMed PMID: 20712824

8. Read J, van Os J, Morrison AP, Ross CA. Childhood trauma, psychosis and schizophrenia: a literature review with theoretical and clinical implications. Acta psychiatrica Scandinavica. 2005;112(5):330-50. doi: 10.1111/1.16000447.2005.00634.x. PubMed PMID: 16223421

9. Wichers M, Schrijvers D, Geschwind N, Jacobs N, Myin-Germeys I, Thiery E, et al. Mechanisms of geneenvironment interactions in depression: evidence that genes potentiate multiple sources of adversity. Psychological medicine. 2009;39(7):1077-86. doi: 10.1017/50033291708004388. PubMed PMID: 18834553.

10. Carpenter LL, Shattuck TT, Tyrka AR, Geracioti TD, Price LH. Effect of childhood physical abuse on cortisol stress response. Psychopharmacology. 2011;214(1):367-75. doi: 10.1007/s00213-010-2007-4. PubMed PMID: 20838776 PubMed Central PMCID: PMC3580170.

11. Heim C, Newport DJ, Bonsall R, Miller AH, Nemeroff CB. Altered pituitary-adrenal axis responses to provocative challenge tests in adult survivors of childhood abuse. The American journal of psychiatry. 2001;158(4):575-81. PubMed PMID: 1128269

12. Teicher MH, Andersen SL, Polcari A, Anderson CM, Navalta CP, Kim DM. The neurobiological consequences of eanly stress and childhood maltreatment. Neuroscience and biobehavioral reviews. 2003;27(1-2):33-44. PubMed PMID: 12732221

13. Hennessy MB, Fitch C, Jacobs S, Deak T, Schiml PA. Behavioral effects of peripheral corticotropin-releasing factor during maternal separation may be mediated by proinflammatory activity. Psychoneuroendocrinology. 2011;36(7):996-1004. doi: 10.1016/j.psyneuen.2010.12.011. PubMed PMID: 21255937; PubMed Central PMCID: PMC3568995.

14. McMillen IC, Robinson JS. Developmental origins of the metabolic syndrome: prediction, plasticity, and programming. Physiological reviews. 2005;85(2):571-633. doi: 10.1152/physrev.00053.2003. PubMed PMID: 15788706.
15. Danese A Moffitt TE, Harrington H. Milne BJ, Polanczyk G, Pariante CM, et al Adverse childhood experiences and adult risk factors for age -related disease: depression inflammation and clustering of metabolic risk markers Archives of pediatrics \& adolescent medicine. 2009:163(12):1135-43. doi: 10.1001/archpediatrics.2009.214. PubMed PMID: 19996051. PubMed Central PMCID: PMC3560401.

16. Nicolson NA, Davis MC, Kruszewski D, Zautra AJ. Childhood maltreatment and diurnal cortisol patterns in women with chronic pain Psychosomatic medicine. 2010:72(5):471-80 doi: 101097/PSY0b013e3181d9a104. PubMed PMID: 20467005.

17. Hamer M, Gibson EL, Vuononvirta R, Williams E, Steptoe A. Inflammatory and hemostatic responses to repeated mental stress: individual stability and habituation over time. Brain, behavior, and immunity. 2006;20(5):456-9. doi: 10.1016/j.bbi.2006.01.001. PubMed PMID: 16488574.

18. Finkelhor D, Turner HA, Shattuck A, Hamby SL. Violence, crime, and abuse exposure in a national sample of children and youth: an update. JAMA pediatrics. 2013;167(7):614-21. doi: 10.1001/jamapediatrics.2013.42. PubMed PMID: 23700186.

9. Vanaelst B, Huybrechts I, De Bourdeaudhuij I, Bammann K, Hadjigeorgiou C, Eiben G, et al. Prevalence of negative life events and chronic adversities in European pre- and primary-school children: results from the 7367-70-26. PubMed PMID: 23173879; PubMed Central PMCID: PMC3552702.

20. Euser S, Alink LR, Pannebakker F, Vogels T, Bakermans-Kranenburg MJ, Van IMH. The prevalence of child maltreatment in the Netherlands across a 5-year period. Child abuse \& neglect. 2013:37(10):841-51. doi: 10.1016/. chiabu.2013.07.004. PubMed PMID: 23938018

21. Dong M, Anda RF, Felitti VJ, Dube SR, Williamson DF, Thompson TJ, et al. The interrelatedness of multiple forms of childhood abuse, neglect, and household dysfunction. Child abuse \& neglect. 2004;28(7):771-84. doi: 10.1016/j. chiabu.2004.01.008. PubMed PMID: 15261471.

22. Glaser JP, van Os J, Portegijs PJ, Myin-Germeys I. Childhood trauma and emotional reactivity to daily life stress in adult frequent attenders of general practitioners. Journal of psychosomatic research. 2006;61(2):229-36. do: 10.1016/j.jpsychores.2006.04.014. PubMed PMID: 16880026.

23. Bunce SC, Larsen RJ, Peterson C. Life after trauma: personality and daily life experiences of traumatized people. Journal of personality. 1995;63(2):165-88. PubMed PMID: 7782991.

24. Lundberg U, Kadefors R, Melin B, Palmerud G, Hassmen P, Engstrom M, et al. Psychophysiological stress and EMG activity of the trapezius muscle International journal of behavioral medicine 1994:1(4):354-70 doi: 10.1207/ s15327558ijbm0104_5. PubMed PMID: 16250795 .

25. Wahlstrom J, Lindegard A, Ahlborg G, Jr., Ekman A, Hagberg M. Perceived muscular tension, emotional stress, psychological demands and physical load during VDU work. International archives of occupational and environmental health. 2003:76(8):584-90. doi: 10.1007/500420-003-0454-5. PubMed PMID: 12898271

26. Luijcks R, Hermens HJ, Bodar L, Vossen CJ, Van Os J, Lousberg R. Experimentally induced stress validated by EMG activity. PloS one. 2014;9(4):e95215. doi: 10.1371/journal.pone.0095215. PubMed PMID: 24736740; PubMed Central PMCID: PMC3988146.

27. Lundberg U, Forsman M, Zachau G, Eklof M, Palmerud G, Melin B, et al. Effects of experimentally induced menta and physical stress on motor unit recruitment in the trapezis muscle. Work Stress. 2002;16(2):166-78. dol: Do 10.1080/02678370210136699. PubMed PMID: WOS:000177065700009.

28. Wijsman J, Grundlehner B, Penders J, Hermens H. Trapezius muscle EMG as predictor of mental stress. Wireles Health 2010; 2010; San Diego, California. 
29. Krantz G, Forsman M, Lundberg U. Consistency in physiological stress responses and electromyographic activity during induced stress exposure in women and men. Integrative physiological and behavioral science : the official journal of the Pavlovian Society. 2004;39(2):105-18. PubMed PMID: 15759598

30. Willmann $\mathrm{M}$, Bolmont $\mathrm{B}$. The trapezius muscle uniquely lacks adaptive process in response to a repeated moderate cognitive stressor. Neuroscience letters. 2012;506(1):166-9. doi: 10.1016/.neulet.2011.10.073. PubMed PMID: 22094381.

31. Dawes RM. The problem of child sexual abuse. Science. 2005;309(5738):1182-5; author reply -5. Epub 2005/08/23. PubMed PMID: 16114119 .

32. Heins M, Simons C, Lataster T, Pfeifer S, Versmissen D, Lardinois M, et al. Childhood trauma and psychosis: a case-control and case-sibling comparison across different levels of genetic liability, psychopathology, and type of trauma. Am J Psychiatry. 2011;168(12):1286-94. Epub 2011/10/01. doi: 10.1176/appi.ajp.2011.10101531. PubMed PMID: 21955935.

33. Myers ND, Brincks AM, Ames AJ, Prado GJ, Penedo FJ, Benedict C. Multilevel modeling in psychosomatic medicine research. Psychosomatic medicine. 2012;74(9):925-36. doi: 10.1097/PSY.0b013e3182736971. PubMed PMID: 23107843; PubMed Central PMCID: PMC3498540

34. Vossen $\mathrm{H}$, Van Breukelen $\mathrm{G}$, Hermens $\mathrm{H}$, Van Os J, Lousberg R. More potential in statistical analyses of event related potentials: a mixed regression approach. International journal of methods in psychiatric research. 2011:20(3):e56-68. doi: 10.1002/mpr.348. PubMed PMID: 21812066.

35. Bromm B, Meier W. The intracutaneous stimulus: a new pain model for algesimetric studies. Methods and findings in experimental and clinical pharmacology. 1984;6(7):405-10. PubMed PMID: 6503475.

36. Jensen C, Vasseljen O, Westgaard RH. The influence of electrode position on bipolar surface electromyogram recordings of the upper trapezius muscle. European journal of applied physiology and occupational physiology. 1993;67(3):266-73. PubMed PMID: 8223542

37. European Network of National Networks studying Gene-Environment Interactions in S, van Os J, Rutten BP Myin-Germeys I, Delespaul P, Viechtbauer W, et al. Identifying gene-environment interactions in schizophrenia: contemporary challenges for integrated, large-scale investigations. Schizophr Bull. 2014;40(4):729-36. do 10.1093/schbul/sbu069. PubMed PMID: 24860087; PubMed Central PMCID: PMCPMC4059449.

38. Woda A, L'Heveder G, Ouchchane L, Bodere C. Effect of experimental stress in 2 different pain condition affecting the facial muscles. J Pain. 2013;14(5):455-66. doi: 10.1016/j.jpain.2012.12.008. PubMed PMID: 23453565.

39. Schleifer LM, Spalding TW, Kerick SE, Cram JR, Ley R, Hatfield BD. Mental stress and trapezius muscle activation under psychomotor challenge: a focus on EMG gaps during computer work Psychophysiology. 2008:45(3):356-

40. Rissén D, Melin B, Sandsjö L, Dohns I, Lundberg U. Psychophysiological stress reactions, trapezius muscle activity, and neck and shoulder pain among female cashiers before and after introduction of job rotation. Work \& Stress. 2002;16(2):127-37. doi: 10.1080/02678370210141530.

41. Nimbarte AD, Al Hassan MJ, Guffey SE, Myers WR. Influence of psychosocial stress and personality type on the biomechanical loading of neck and shoulder muscles. International Journal of Industrial Ergonomics. 2012;42(5):397-405. doi: http://dx.doi.org/10.1016/j.ergon.2012.05.001.

42. Bal S, Van Oost P, De Bourdeaudhuij I, Crombez G. Avoidant coping as a mediator between self-reported sexual abuse and stress-related symptoms in adolescents. Child abuse \& neglect. 2003;27(8):883-97. PubMed PMID:
43. Min M, Farkas K, Minnes S, Singer LT. Impact of childhood abuse and neglect on substance abuse and psychological distress in adulthood.J Trauma Stress. 2007:20(5):833-44 doi: 101002/jts 20250. PubMed PMID: 17955535

44. Krause ED, Mendelson T, Lynch TR. Childhood emotional invalidation and adult psychological distress: the mediating role of emotional inhibition. Child abuse \& neglect. 2003;27(2):199-213. PubMed PMID: 12615094.

45. Nickel R, Egle UT. Coping with conflict as pathogenetic link between psychosocial adversities in childhood and psychic disordersinadulthood.ZPsychosom Med Psyc.2001-47(4):332-47.PubMed PMID.WOS.000172348100003. 


\section{APPENDIX A}

\section{Example used multilevel mode}

MIXED Log_RMS_left WITH age sex linear_time quadratic_time exponential_time

$$
\text { ACE_SCORE session }
$$

/CRITERIA=CIN(95) MXITER(100) MXSTEP(10) SCORING(1) SINGULAR(0.000000000001) HCONVERGE(0, ABSOLUTE) LCONVERGE(0, ABSOLUTE) PCONVERGE(0.000001, ABSOLUTE)

/FIXED= age sex linear_time quadratic_time exponential_time ACE_SCORE session linear_time*ACE_SCORE quadratic_time*ACE_SCORE

exponential_time*ACE_SCORE ISSTYPE(3)

METHOD=REML

PRINT=SOLUTION TESTCOV

/RANDOM=INTERCEPT | SUBJECT(subject) COVTYPE(UN)

/RANDOM=INTERCEPT linear_time | SUBJECT(subject*session) COVTYPE(UN)

/REPEATED = time_within_subject | SUBJECT(subject*session) COVTYPE(AR1).

\section{APPENDIX B}

Multilevel model, EMG activity of the left trapezius muscle served as the dependent.

$T$ - and $p$-values of the time*ACE-score interaction effects are presented in Table 1.

\section{Estimates of Fixed Effects}

\begin{tabular}{|c|c|c|c|c|c|c|c|}
\hline \multirow{3}{*}{ Parameter } & \multirow{3}{*}{ Estimate } & \multirow{3}{*}{ Std. Error } & \multirow[b]{3}{*}{ df } & \multirow{3}{*}{$\mathrm{t}$} & \multirow[b]{3}{*}{ Sig. } & \multicolumn{2}{|c|}{ 95\% Confidence Interva } \\
\hline & & & & & & Lower & Upper \\
\hline & & & & & & & \\
\hline Intercept & 1,478384 & 0,084595 & 151,701 & 17,476 & 0 & 1,311249 & 1,64552 \\
\hline Age & $-0,002818$ & 0,001489 & 94,437 & $-1,893$ & 0,061 & $-0,005773$ & 0,000138 \\
\hline Sex & 0,008781 & 0,052457 & 97,31 & 0,167 & 0,867 & $-0,095327$ & 0,112888 \\
\hline Exponential time & $-0,002724$ & 0,001604 & 1514,048 & $-1,698$ & 0,09 & $-0,005871$ & 0,000423 \\
\hline Quadratic time & $1,28 \mathrm{E}-05$ & $4,56 \mathrm{E}-06$ & 2533,21 & 2,814 & 0,005 & 3,89E-06 & $2,18 \mathrm{E}-05$ \\
\hline Linear time & $-0,00116$ & 0,000383 & 420,972 & $-3,033$ & 0,003 & $-0,001912$ & $-0,000408$ \\
\hline Experimental session & 0,025834 & 0,029733 & 82,721 & 0,869 & 0,387 & $-0,033306$ & 0,084974 \\
\hline CHILDABUSE_ALL & $-0,008049$ & 0,009109 & 107,992 & $-0,884$ & 0,379 & $-0,026105$ & 0,010007 \\
\hline $\begin{array}{l}\text { Quadratic_time* } \\
\text { CHILDABUSE_ALL }\end{array}$ & $-5,00 \mathrm{E}-06$ & $1,10 E-06$ & 2534,554 & $-4,548$ & 0 & $-7,15 \mathrm{E}-06$ & $-2,84 \mathrm{E}-06$ \\
\hline $\begin{array}{l}\text { Exponential_time * } \\
\text { CHILDABUSE_ALL }\end{array}$ & 0,001418 & 0,000387 & 1515,033 & 3,668 & 0 & 0,00066 & 0,002176 \\
\hline $\begin{array}{l}\text { Linear_time* } \\
\text { CHILDABUSE_ALL }\end{array}$ & 0,00032 & $9,21 E-05$ & 421,179 & 3,475 & 0,001 & 0,000139 & 0,000501 \\
\hline
\end{tabular}

Dependent Variable: Log_RMS_left. 


\section{Chapter 4}

The influence of perceived stress on cortical reactivity: a proof-of-principle study

Luijcks R', Vossen $\mathrm{CJ}^{1,2}$, Hermens $\mathrm{HJ}^{3}$, van Os J ${ }^{1,4}$ and Lousberg R

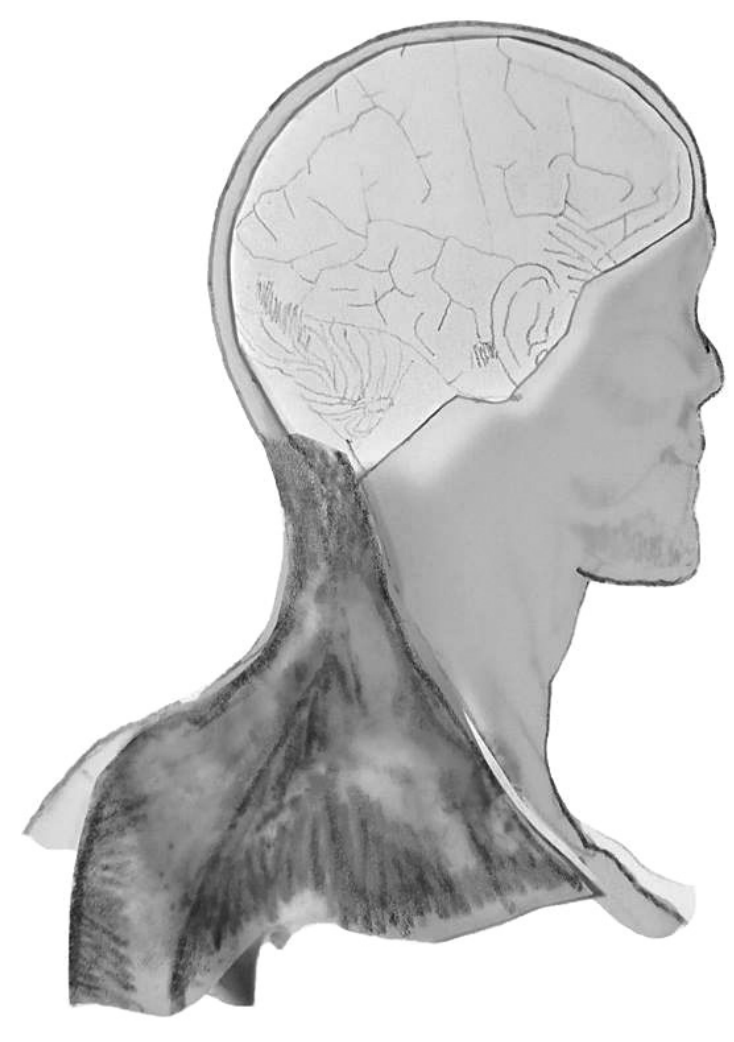

1 Department of Psychiatry \& Psychology, Maastricht University Medical Centre, Maastricht, The Netherlands. 2 Department of Anesthesiology and Pain Medicine, Maastricht University Medical Centre, Maastricht, The Netherlands.

3 Roessingh Research and Development, Enschede, The Netherlands.

4 King's College London, King's Health Partners, Department of Psychosis Studies, Institute of Psychiatry, London, United Kingdom 


\section{ABSTRACT}

Theaimofthisstudywastoinvestigatehowperceivedstressmayaffectelectroencephalographica (EEG) activity in a stress paradigm in a sample of 76 healthy participants. EEG activity was analyzed using multilevel modeling, allowing estimation of nested effects (EEG time segments within subjects). The stress paradigm consisted of a 3-minute pre-stimulus stress period and a 2-minute post-stimulus phase. At $t=3$ minutes, a single electrical stimulus was delivered. Participants were unaware of the precise moment of stimulus delivery and its intensity level. In the EEG time course of alpha activity, a stronger increase was observed during the poststimulus period as compared to the pre-stimulus period. An opposite time course effect was apparent for gamma activity. Both effects were in line with a priori expectations and support the validity of this experimental EEG-stress paradigm.

Secondly, we investigated whether interaction effects of stress and coping, as measured with the Perceived Stress Scale-10 questionnaire (PSS-10), could be demonstrated.

A higher perceived stress score was accompanied by a greater increase in delta- and thetaactivity during the post-stimulus phase, compared to low scores. In contrast, low coping capacity was associated with a stronger decrease in slow beta, fast beta and gamma activity during the post-stimulus phase.

The results of the present article may be interpreted as proof-of-principle that EEG stressrelated activity depends on the level of subjectively reported perceived stress. The inclusion of psychosocial variables measuring coping styles as well as stress-related personality aspects permits further examination of the interconnection between mind and body and may inform on the process of transformation from acute to chronic stress.

\section{INTRODUCTION}

Psychological and physical sequelae of stress are common. Experience of stress is accompanied by an increased level of arousal and may lead to a number of physiological reactions, such as acceleration of heart rate, pupil dilatation, increased galvanic skin response and increased finger pulse volume [1]. Increased muscle activity, especially in the trapezius muscles, and thus an increased EMG response is also associated with stress [2]. EMG activity can be viewed as a peripheral correlate of stress, in the same way as brain activity may represent a central correlate of stress. Cortical activity in relation to stress can be studied using a range of dependent variables, such as electroencephalography (EEG), PET-scan or (f)MRI. In the present study, we chose to investigate the effects of experimentally induced stress on EEG activity. The main reason to focus on EEG as a stress-related variable is the possibility to model the dynamic time aspect of cortical activity.

A number of studies have investigated associations between EEG activity and stress [3-7]. Within these lines of research, in which stress has been experimentally manipulated, there is not only a focus on frontal alpha activity, but also on the left and right hemisphere asymmetry $[3,5,8,9]$. It is assumed that the left hemisphere is more involved in the processing of positive emotions, whereas the right hemisphere is associated with the processing of negative emotions [9]. Most studies report a relatively higher alpha activity in relaxation states compared to stressful situations [3,5,8,10,11]. In addition, stressful conditions like anxiety disorder [12] may be associated with alterations in EEG gamma activity $[10,13]$. Subsequently, relatively lower alpha activity and relatively higher gamma activity can be expected during a stressful period. As to lateralization effects, since stress can be viewed as negative emotion, the effects of stress on the alpha and gamma band may be more pronounced in the right hemisphere.

Most stress experiments include a mental stress task. However, performing such a task may interfere with stress-related cortical processes in the EEG. The challenge in this type of research is to differentiate between cortical activity associated with performing the task itself and the cortical stress component. In response to this methodological problem, a novel experimental stress paradigm was recently developed. In this paradigm, one single unpredictable and uncontrollable electrical stimulus is presented, thus inducing both a distinct cognitive stressor and a physical stressful stimulus. The electrical stimulus divides the experimental phase into two periods: a pre- and a post-stimulus period. An anticipatory stress effect, a direct response to the nociceptive stimulus and a subsequent recovery to baseline levels were demonstrated for EMG activity [2]. In the latter study, it was demonstrated that EMG activity increased in the anticipatory pre-stimulus phase, followed by a decrease in EMG activity after the delivery of the stimulus. Additionally, it was shown that higher subjective stress was associated with a higher level of muscle activity. This new developed paradigm, validated with a subjective 
stress measure and with EMG, can be used to investigate the effects of experimentally induced stress on brain activity, examined by EEG.

The main goal of the present article is to further investigate the relationship between the bands of the full frequency EEG spectrum and experimentally induced stress. Differential cortical processing is expected between the anticipatory pre-stimulus period and the poststimulus period of the novel design. Since stress is a phenomenon that can vary over time, stress-related EEG activity is expected to vary over time as well. Therefore, multilevel regression is required to model the within-condition EEG time effects. The average EEG effect of induced stress can be considered as a state, and gives no information about ongoing processes. The use of multilevel modeling provides insight in time-related EEG processing and may therefore lead to more informed conclusions. Thus, two a priori hypotheses were examined: (i) a relatively larger increase in alpha-activity and (ii) a relatively larger decrease in gamma activity were expected during the post-stimulus phase, compared to the stressful pre-stimulus phase. With respect to location effects, no a priori hypotheses were formulated, since these analyses pertain to the time course of the EEG activity, instead of absolute pre-post activity. In addition, a similar argumentation can be offered for lateralization effects.

Additionally, we were interested to investigate the interaction effect between subjective stress experience and the EEG time course. In the study on experimentally induced stress and its association with EMG activity[2], such a relation was demonstrated. It may be hypothesized that the level of perceived stress in the preceding weeks impacts brain activity during the experiment. This hypothesis is not only intuitive from a clinical point of view, but is also supported by experimental research [4]. In order to take this source of variation into account, the 10-item Perceived Stress Scale (PSS-10), comprising two subscales (stress and coping), was used [14].

\section{MATERIALS AND METHOD}

\section{Ethics Statement}

The study was conducted according to the principles of the Declaration of Helsinki and was approved by the ethics committee of the Academic Hospital Maastricht and Maastricht University (METC azM/UM, Maastricht). Before the start of the experiment, subjects provided written informed consent.

\section{Subjects}

Seventy-six right-handed subjects (46 females and 30 males) participated in the study. Their age ranged from 18 to 65 years. Exclusion criteria were structural use of antipsychotics, anti- epileptics or anxiolytics during the past year or structural use of alcohol (>10 u/day). Subjects were asked to refrain from alcohol-containing consumptions the evening before and to refrain from caffeine-containing consumptions three hours prior to the experiment.

\section{Electroshocker and stimuli}

An electro-shocker (type Shocko-100-AA-20, developed by Maastricht Instruments BV and approved for usage in experimental studies) was used to deliver electrical stimuli (see also [15]). Stimuli were electrical pulses of 10 milliseconds duration, administered intracutaneously on the top of the middle finger of the non-dominant left hand, as described by Bromm and Meier [16]. The sensation and pain threshold were determined by gradually increasing the intensity of the stimulus, starting at zero intensity. The first intensity that was consciously experienced was defined as the sensation threshold, the first intensity experienced as painful was defined as the pain threshold. This procedure was repeated three times in order to obtain a reliable estimate. The intensity of the electrical stimulus applied during the experiment was computed for each subject individually. The intensity of the actually delivered stress stimulus during the experiment was calculated as follows:

Actually delivered stress stimulus $=$ pain threshold $+0.25 *$ (pain threshold - sensation threshold)

As shown in a previous experiment, this intensity level was experienced as painful by all subjects, albeit still acceptable [15].

\section{Procedure}

EEG-electrodes as well as the shock electrode were attached, as described below. Next, a baseline measurement of 3 minutes was conducted. After determination of the individual pain threshold, subjects were instructed that they would receive a single electrical shock over a 5 minute period. The experimenter pointed out that the precise moment of stimulus delivery and its intensity level would be determined by a personal computer. In addition, participants were told that stimulus intensity might vary between the sensation threshold and a level clearly above the pain threshold. Subjects were instructed to keep both hands on the table, palms down, and not to close their eyes during the whole measurement period. In fact, all subjects received the experimental stimulus on exactly $t=3$ minutes. The whole procedure was controlled by the software program "Presentation 0.71" (Neurobehavioral Systems).

\section{Psychophysiological recordings}

All recordings were conducted in an electrically and sound-shielded cubicle (7,1 m2). Ag/AgCl electrodes were placed on 14 different locations (Fz, F3, F4, Cz, C3, C4, Pz, P3, P4, T3, T4, Oz, 
$\mathrm{O} 1$ and O2), using the international 10-20 system [17]. Impedances were kept below $5 \mathrm{k} \Omega$. A reference electrode was placed on each ear lobe. To control for vertical eye movements, an EOG electrode was placed $1 \mathrm{~cm}$ under the midline of each eye. A ground electrode was placed at Fpz. All electrodes were fixed using 10-20 conductive past. Brainvision BrainAmp Research Amplifier (Brain Products; sampling rate $1000 \mathrm{~Hz}$, resolution $0.1 \mu \mathrm{V}$ ) was used for EEG recording.

\section{Offline dataprocessing}

EEG was recorded online with $1000 \mathrm{~Hz}$ sampling rate, using Brainvision 2.0. Data was offline filtered (bandpass $0.5-50 \mathrm{~Hz}$ ) and segmented into epochs of $512 \mathrm{~ms}$, without overlapping segments and using a Hamming window followed by a Fast Fourier transformation (FFT). The EEG spectrum was divided into frequency bands, which were defined as follows: delta $(1-4 \mathrm{~Hz})$, theta $(4-8 \mathrm{~Hz})$, alpha $(8-13 \mathrm{~Hz})$, slow beta $(13-20 \mathrm{~Hz})$, fast beta $(20-30 \mathrm{~Hz})$ and gamma $(30-50 \mathrm{~Hz})$. Epochs with EOG activity exceeding $+100 \mathrm{mV}$ and $-100 \mathrm{mV}$ were excluded from the analyses.

\section{Psychological measurements}

The 10-item Perceived Stress Scale was used to assess subjective stress over the past month The PSS-10 has been translated into different languages and its validity has been demonstrated in several populations [18-20]. The two-factor structure of the PSS-10 was used, since there is a conceptual difference between both factors: the stress factor and the coping factor [21].

Psychometric analyses were performed. Factor analysis showed a two-factor structure, consisting of a 6 -item stress factor and a 4-item coping factor. Crohnbach's alpha's for these two factors were 0.811 and 0.812 respectively. The 4 items that accounted for the coping factor were inverted. Consequently, a high score for the coping factor means that the coping capacity is low.

\section{Statistical analysis}

Because of the hierarchical structure of the current EEG dataset, consisting of epochs (level 1) that are clustered within individuals (level 2), multilevel regression analyses were performed. In all multilevel regression analyses, EEG activity served as the dependent variable. In order to obtain normality, all dependent variables, consisting of the different EEG bands at different locations, were log transformed. Epoch number and condition (pre- versus post-stimulus period), as well as the covariates age and sex, served as independent variables in a basic model. The predictor of main interest was the interaction between the time variable (epoch number) and condition.
In order to discover which covariance structure yielded the best fit for the dataset, various covariance structures were tested. The -2 log likelihood of different models was calculated in order to determine which statistical model would fit best. An autoregression (AR1) structure turned out to be significantly better (lowest -2 log likelihood) than that of its competitors, namely compound symmetry (CS) and Scaled Identity. Since the dataset of EEG activity is composed as a multilevel data file, consisting of consecutive epochs, each epoch is correlated with the previous epoch, which makes an autoregression model convenient. The AR1 structure was therefore used for all statistical analyses. All models were executed with a random intercept. All statistical analyses were performed using SPSS 21.0. P-values equal to or below 0.05 were considered to be statistically significant.

\section{RESULTS}

Due to protocol violations (subjects who closed their eyes during the experiment, subjects who did not follow the instructions), 7 subjects were excluded from the analyses, leaving $n=69$ analyzable participants (40 females, 29 males). Age ranged from 18 to 65, with a mean age of 36 years. The scores on the PSS-10 ranged from 2 to 24 (with a maximum of 40 points). For all subjects, the difference between the pain and sensation threshold was calculated. The average value of the sensation threshold was $0.35 \mathrm{~mA}(\mathrm{SD}=$ range $0.10 \mathrm{~mA}$ to $1.05 \mathrm{~mA}$ ); the average pain threshold was $1.37 \mathrm{~mA}(\mathrm{SD}=$ range $0.15 \mathrm{~mA}$ to $3,65 \mathrm{~mA}$ ); the average of all calculated differences in pain threshold and sensation threshold was 1,02 $\mathrm{mA}(\mathrm{SD}=$ range 0.05 $\mathrm{mA}$ to $3.2 \mathrm{~mA}$ ).

\section{EEG time course effects within the stress experiment}

Since no a priori hypotheses were formulated with respect to location effects, overall analyses of the spectral power time course were executed for all 6 EEG power bands in order to examine robust effects. For illustrational purposes, a graph of the predicted estimated EEG time course during the experiment was computed for the alpha and gamma activity band. With respect to the alpha activity, Fig. 1 shows that the increasing trend in spectral power is more apparent in the post-stimulus period compared to the pre-stimulus period. For gamma activity, an opposite effect can be observed: in both the pre- and post-stimulus period, a decreasing trend is present (Fig. 2). The significant negative t-value of -3.91 (as can be seen in Table 1) can be interpreted as a stronger decrease in gamma activity in the post-stimulus period. An overall analysis of the time* condition interaction was executed for all 6 EEG bands. As shown in Table 1, there was a significant positive interaction effect for the alpha and fast beta band, indicating a relatively stronger increase during the post-stimulus phase compared to the pre-stimulus phase. The significant negative interaction effect on the gamma band should be interpreted in the opposite way. 


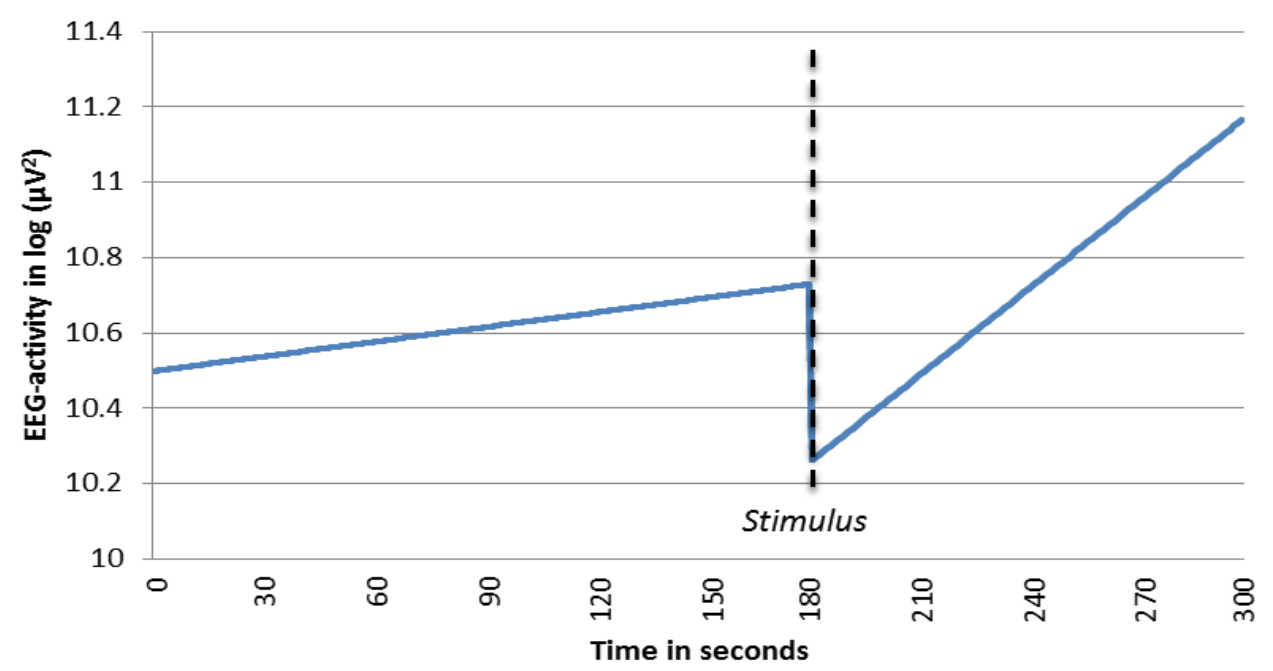

Fig. 1. Fitted time course of spectral power of the alpha band for pre- and post-stimulus period. Not specified per location. Stimulus delivery took place on exactly $t=180$ seconds. The increasing trend in spectral power is more apparent in the post-stimulus period compared to the pre-stimulus period.

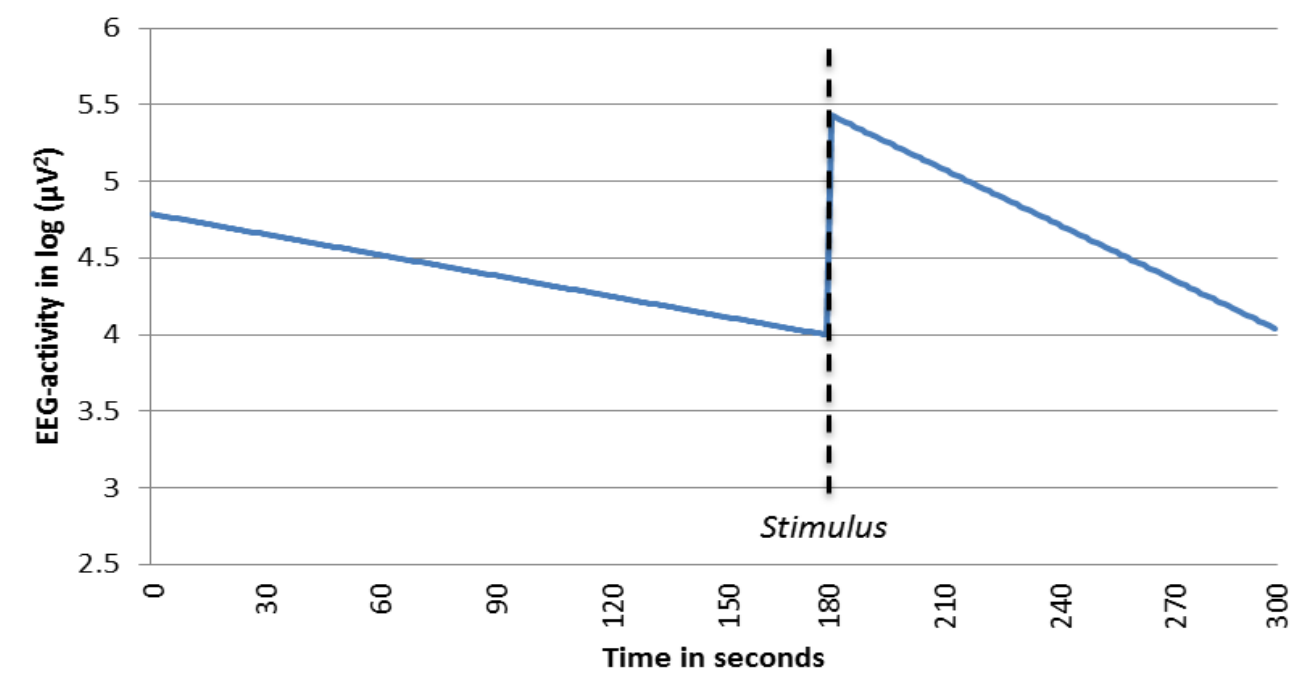

Fig. 2. Fitted time course of spectral power of the gamma band for pre- and post-stimulus period. Not specified per location. Stimulus delivery took place on exactly $t=180$ seconds. In both the pre- and post-stimulus period, a decreasing trend is present; nevertheless, a significant stronger decrease of gamma activity in the post-stimulus period is apparent.
Table 1. Multilevel analyses of time* condition interactions on all 6 EEG-bands

\begin{tabular}{lll} 
Band & T-value & P-value \\
\hline Delta & $-0,80$ & 0.42 \\
Theta & $-0,49$ & 0.62 \\
Alpha & 3,74 & $<0.01$ \\
Slow beta & 4,18 & $<0.01$ \\
Fast beta & $-0,33$ & 0.74 \\
Gamma & -3.91 & $<0.01$ \\
\hline
\end{tabular}

Values shown in table 1 are T-values and p-values of the time*condition interaction effects. Condition was coded as 0 and 1 , contrasting the pre- and post-stimulus period. Significant time*condition interaction effects were found for the alpha, slow beta and gamma band. The negative T-value for gamma activity can be interpreted as a stronger decrease in gamma activity in the post-stimulus period.

In addition, the same models were run for each of the 14 locations separately. As was expected from the (location) aggregated band analyses, for nearly none of the locations a time* condition interaction effect could be demonstrated on the delta and theta bands (Table 2). With respect to alpha and slow beta activity, the strongest effects were seen in the central and parietotemporal area; no robust effects could be detected in the frontal and occipital area. Inspection of these results suggests a lateralization effect, the right side having stronger interaction effects than the left side. Additionally, for the fast beta band there are also significant interaction effects. However, there were both positive and negative interaction effects, which could be a reason for not finding an overall band effect (see previous paragraph). Finally, a significantly much higher significant decrease in gamma activity in the post-stimulus period compared to the pre-stimulus period was found, especially in the frontal, central and occipital regions. 
Table 2. T-values for the time* ${ }^{*}$ condition interaction effects on all $6 \mathrm{EEG}$-bands, per location

$$
\begin{array}{llllllllllllll}
\mathrm{Fz} & \mathrm{F} 3 & \mathrm{~F} 4 & \mathrm{Cz} & \mathrm{C} 3 & \mathrm{C} 4 & \mathrm{Pz} & \mathrm{P} 3 & \mathrm{P} 4 & \mathrm{Oz} & \mathrm{O} 1 & \mathrm{O} 2 & \mathrm{T3} & \mathrm{T} 4
\end{array}
$$

$\begin{array}{lllllllllllllll}\text { Delta } & -1,53 & -1,49 & -1,81 & -0,59 & -0,74 & -0,57 & -0,90 & -0,48 & 0,70 & -0,22 & 0,16 & -0,06 & -0,95 & 0,32\end{array}$

$\begin{array}{lllllllllllllll}\text { Theta } & -1,80 & -2,05^{*} & -1,81 & -0,30 & -0,80 & 0,13 & 1,13 & 1,00 & 1,60 & -0,47 & -0,06 & -0,23 & 0,35 & 1,37\end{array}$

Alpha $\quad 2,49^{*} \quad 0,65 \quad 1,44 \quad 4,06^{*} \quad 1,87 \quad 5,07^{*} \quad 4,75^{*} \quad 3,60^{*} \quad 5,53^{*} \quad 1,30 \quad 4,18^{*} \quad 2,85^{*} \quad 2,73^{*} \quad 3,95^{*}$

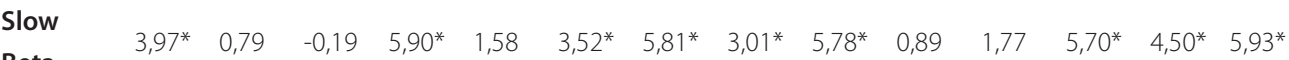

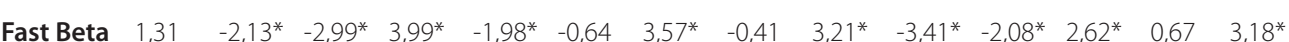

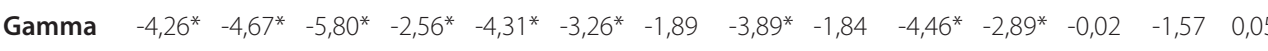

Values shown are T-values, demonstrating the significant time ${ }^{*}$ condition interaction effects per location for all frequency bands. Condition was coded as 0 and 1, contrasting the pre- and post-stimulus period. Results with corresponding P-values $\leq 0.05$ were considered to be significant (all significant results were marked with *). Negative T-values can be interpreted as a stronger decrease in EEG activity in the post-stimulus period.

\section{The effect of subjective stress on the EEG time course}

For all EEG frequency bands, the interaction between the PSS-10 and the EEG time course during the experiment was examined, contrasting the pre-stimulus period and the post-stimulus period.

\section{Stress factor}

The stress component of the PSS-10 had a significant effect on the delta and theta condition*time interaction (Table 3). These results indicate that with a higher perceived stress score, a more pronounced increase in delta- and theta-activity exists during the post-stimulus phase when compared to the pre-stimulus phase.

When specifying for location, taking into account all 14 locations, a frontal and central effect can be seen for theta activity (Table 4). Also, this PSS time course interaction effect appeared to be related to the left hemisphere (F3, C3, P3, O1, T3). There was a relatively strong PSS time course interaction effect on fast beta and gamma activity in the temporal area, as can be seen in table 4 .
Table 3. T-values for the time*condition*subjective stress interactions on all 6 EEG-bands

\begin{tabular}{lllll}
\hline Bands & \multicolumn{2}{l}{ PSS Coping } & \multicolumn{2}{l}{ PSS Stress } \\
\hline & T-value & P-value & T-value & P-value \\
Delta & -0.54 & 0.59 & 1.96 & 0.05 \\
Theta & -1.83 & 0.07 & 2.74 & 0.01 \\
Alpha & -1.80 & 0.07 & -1.39 & 0.17 \\
Beta slow & -2.31 & 0.02 & 0.96 & 0.34 \\
Beta fast & -3.01 & $<0.01$ & -0.46 & 0.65 \\
Gamma & -2.25 & 0.02 & -1.16 & 0.24 \\
\hline
\end{tabular}

Values shown are T-values and their corresponding p-values, demonstrating the time*condition interaction effects. Condition was coded as 0 and 1 contrasting the pre- and post-stimulus period. Subjective stress was defined by the T. values can be interpreted as a stronger decrease in EEG activity in the post-stimulus period.

Table 4. T-values for the time*condition*PSS stress interactions on all 6 EEG-bands, per location

$$
\begin{array}{llllllllllllll}
\mathrm{Fz} & \mathrm{F} 3 & \mathrm{~F} 4 & \mathrm{Cz} & \mathrm{C} 3 & \mathrm{C} 4 & \mathrm{Pz} & \mathrm{P} 3 & \mathrm{P} 4 & \mathrm{Oz} & \mathrm{O} 1 & \mathrm{O} 2 & \mathrm{~T} 3 & \mathrm{~T} 4
\end{array}
$$

$\begin{array}{lllllllllllllll}\text { Delta } & 2.87^{*} & 1.93 & 3.18^{*} & 1.93 & 1.46 & 2.47^{*} & 1.16 & 1.23 & 1.73 & 1.96^{*} & 1.16 & 1.43 & 1.66 & 1.85\end{array}$

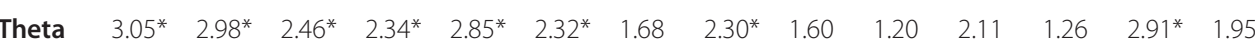

$\begin{array}{lllllllllllllll}\text { Alpha } & 0.32 & 0.20 & 0.45 & -1.35 & -1.64 & -0.45 & -2.62^{*} & -1.97^{*} & -1.20 & -0.88 & -1.75 & -1.30 & -1.04 & -0.88\end{array}$

$\begin{array}{lllllllllllllll}\text { Beta } & 1.86 & 2.24^{*} & 1.60 & 0.85 & -0.31 & 0.86 & -0.22 & -0.58 & 0.71 & 1.31 & 1.60 & 1.96^{*} & -2.29^{*} & 0.91\end{array}$

slow $1.86-2.24 * 1.60-0.85$

$\begin{array}{lllllllllll}\text { fast } & 0.70 & 2.52^{*} & 1.34 & -0.02 & -1.73 & 0.57 & -0.21 & -2.87 & 0.65 & 1.35\end{array}$

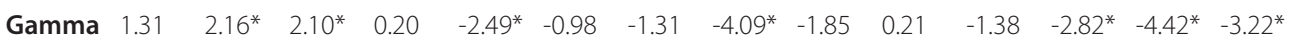

Values shown are T-values, demonstrating the time*condition*PSS stress interaction effects per location for all

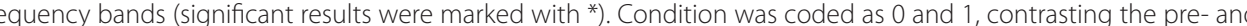
post-stimulus period. Negative T-values can be interpreted as a stronger decrease in EEG activity in the post-stimulus period. 


\section{Coping factor}

The coping factor of the PSS 10 shows a relation with slow and fast beta activity as well as with gamma activity. The results of the multilevel analyses show that with a low coping capacity (i.e. high score on the questionnaire), a stronger decrease over time takes place in slow beta, fast beta and gamma activity during the post-stimulus phase, in contrast with the pre-stimulus phase. Figures of the predicted estimated EEG time course were computed for the fast beta and gamma band (Fig 3. and Fig. 4).

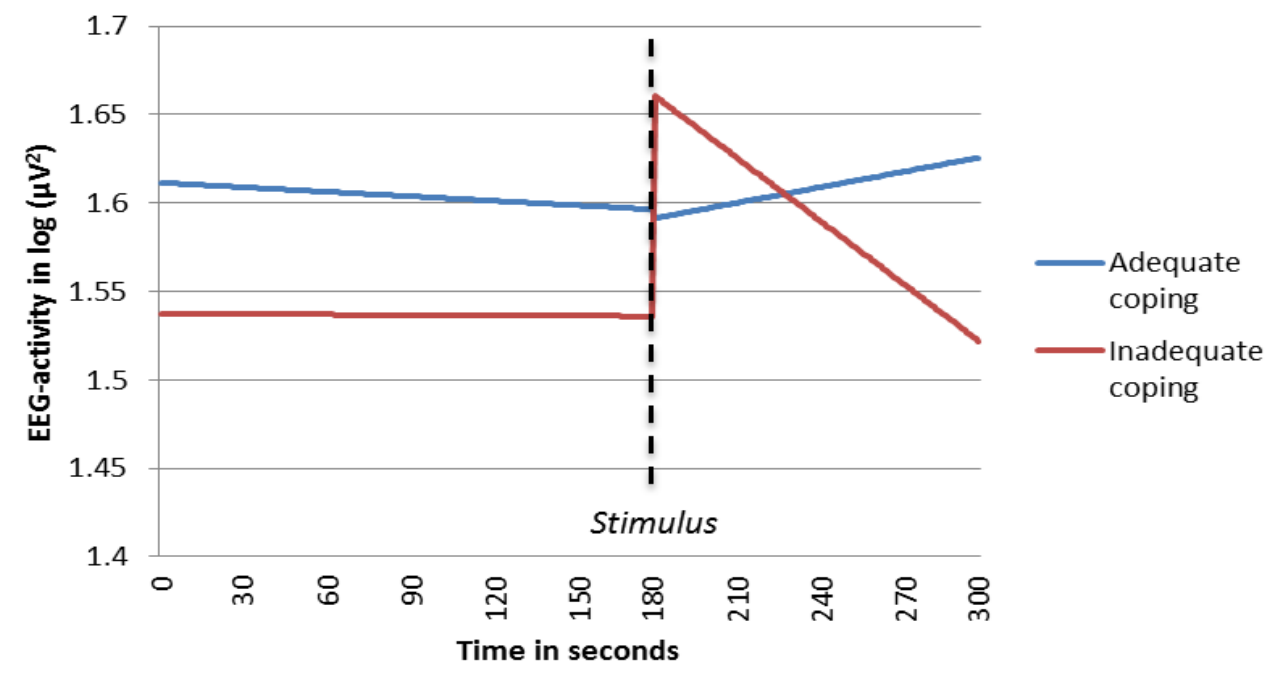

Fig. 3. Fitted time course of spectral power of the fast beta band, interacted with coping capacity. Adequate coping and inadequate coping are contrasted. With a low coping capacity (i.e. high score on the questionnaire), a stronger decrease over time takes place in the post-stimulus period for spectral power of the fast beta band.

As shown in Table 3, the coping component of the PSS-10 has a significant effect on the time course of the complete beta band and the gamma activity. Table 5 depicts a specification for the 14 cranial locations. As can be seen, the time*coping interaction effect on slow beta has a trend on many locations, but this trend is only significant in parietal and temporal areas. On fast beta activity as well as on gamma activity there are significant effects on almost all locations. Additionally, there is also a clear significant effect on frontal alpha activity, which could not be demonstrated in the aggregated band analyses. It appears that with lower coping capacity alpha activity decreases more in the post-stimulus phase when compared to the pre-stimulus phase.

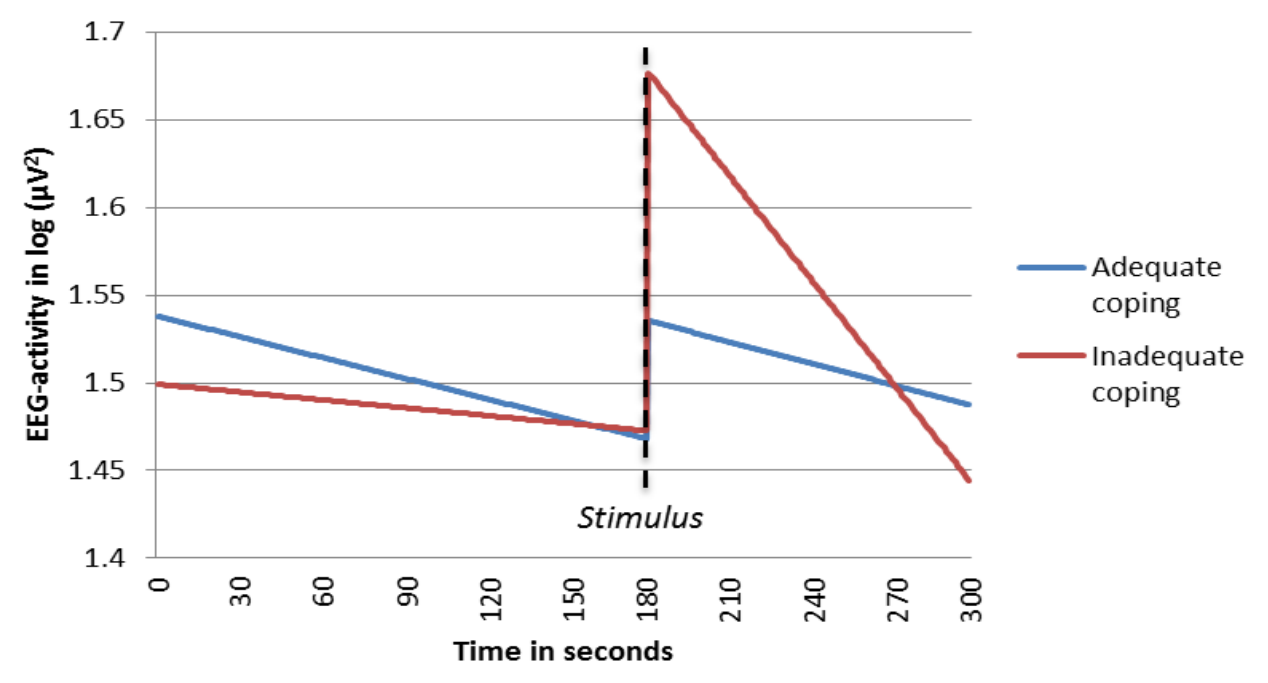

Fig. 4. Fitted time course of spectral power of the gamma band, interacted with coping capacity. Adequate coping and inadequate coping are contrasted. With a low coping capacity (i.e. high score on the questionnaire), a stronger decrease over time takes place in the post-stimulus period for spectral power of the gamma band.

Table 5. T-values for the time*condition*PSS coping interactions on all 6 EEG-bands, per location

\begin{tabular}{lllllllllllllll}
\hline & Fz & F3 & F4 & Cz & C3 & C4 & Pz & P3 & P4 & Oz & $\mathbf{0 1}$ & $\mathbf{0 2}$ & T3 & T4 \\
\hline Delta & 0.03 & -0.95 & 0.76 & 0.90 & -0.45 & 0.90 & 0.20 & 0.10 & 0.67 & -0.36 & -1.11 & -0.18 & -0.71 & 1.20 \\
Theta & $-2.00^{*}$ & -1.24 & -1.90 & -1.56 & -0.89 & -1.50 & -1.05 & -0.39 & -1.88 & -1.94 & $-2.08^{*}$ & $-2.40^{*}$ & -0.54 & -1.52 \\
Alpha & $-2.52^{*}$ & $-2.37^{*}$ & $-2.19^{*}$ & -1.80 & $-2.11^{*}$ & -1.32 & -1.14 & -1.76 & -0.90 & -0.54 & -1.60 & -0.86 & -1.91 & -1.19 \\
Beta slow & -1.60 & -1.70 & -1.72 & -1.53 & -1.80 & $-2.56^{*}$ & -1.18 & $-2.22^{*}$ & $-2.68^{*}$ & -0.55 & -1.31 & -1.54 & $-2.49^{*}$ & $-2.57^{*}$ \\
Beta fast & $-2.33^{*}$ & $-2.37^{*}$ & -1.34 & -1.23 & $-2.61^{*}$ & $-2.15^{*}$ & $-2.83^{*}$ & $-3.92^{*}$ & $-3.50^{*}$ & $-2.12^{*}$ & $-2.09^{*}$ & $-3.42^{*}$ & $-3.77^{*}$ & $-3.31^{*}$ \\
Gamma & $-2.62^{*}$ & $-2.19^{*}$ & -0.62 & $-2.54^{*}$ & $-2.76^{*}$ & -1.73 & $-2.09^{*}$ & $-3.00^{*}$ & $-2.45^{*}$ & -0.98 & -0.62 & $-2.99^{*}$ & $-3.34^{*}$ & $-2.72^{*}$ \\
\hline
\end{tabular}

Values shown are T-values, demonstrating the time*condition*PSS coping interaction effects per location for all frequency bands (significant results were marked with*). Condition was coded as 0 and 1 , contrasting the pre-and poststimulus period. Negative T-values can be interpreted as a stronger decrease in EEG activity in the post-stimulus period. 


\section{DISCUSSION AND CONCLUSIONS}

This is, to the best of our knowledge, the first study investigating how the time course of stress-related EEG activity can be modeled using multilevel regression analysis. Based on evidence reported in experimental stress studies, we formulated expected directions of EEG time* ${ }^{*}$ condition interaction effects for alpha and gamma activity. Further, we examined whether and how subjective stress impacted these time*condition interaction effects, for both the stress factor and the coping factor of the PSS-10.

\section{EEG time course within the stress experiment}

With respect to the EEG time course of alpha activity pre- and post-stimulus, alpha activity appeared to increase more during the post-stimulus period, than the pre-stimulus period as a priori expected. This interaction effect may be considered obvious, since subjects were told that after experiencing one stimulus, no other stimulus would be delivered. The poststimulus period can therefore be conceptualized as a phase in which the arousal effect from the induced stressor fades away.

A similar argumentation can be given for the a priori expected larger decreasing effect of gamma activity in the post-stimulus period. The decrease of gamma activity in the present experiment is consistent with findings in previous research, in which an increase in gamma activity was associated with experimentally induced stress in anxious people [8][22]. It is remarkable that most of the significant gamma effects were found in the frontal, central and temporal regions. In addition to the a priori hypothesized effects, an increasing effect in the slow beta band was observed. Before interpreting this effect, a next step would be to replicate this post-hoc finding.

The PSS-10 was used to examine whether the EEG time course is influenced by perceived stress and coping capacity. No a priori hypotheses were formulated with respect to bands, locations and direction of time effects. Significant interaction effects between the PSS-10 subscales and the EEG time course were demonstrated. Interestingly, the coping and stress subscales showed different effects on the time course of spectral power. Concerning the stress factor of the PSS-10, a higher perceived stress score was accompanied by a greater increase in deltaand theta-activity during the post-stimulus phase, compared to the pre-stimulus phase. In contrast, the analyses of the coping factor of the PSS-10 showed that a low coping capacity was associated with a greater decrease in slow beta, fast beta and gamma activity during the post-stimulus phase. In case of aggregating all cranial locations, no interaction effects of the coping factor of the PSS-10 on the time course of alpha activity could be demonstrated. However, when analyzing all locations separately, significant effects on frontal alpha activity stand out.
The results of earlier studies can be brought to bear on the interpretation of the interaction effects between PSS and the time course of the spectral power of delta and theta bands. First, Klimesch et al. reported that increased theta activity is associated with the encoding of new information and recall of episodic memory [23,24]. In these articles, it was demonstrated that the highest amount of relative theta power was apparent in the frontal and central areas. This is in line with the results of the present study. Second, it is known that stressful or emotionally arousing events can activate memory $[25,26]$. Based on these findings, the following post hoc explanation is postulated: the increased theta-activity during the post-stimulus phase for people with a higher stress score may correspond to a recall of episodic memory, and thus to an evaluation of the stressor. Another post hoc explanation is based on previous studies, in which the authors report an increase in delta and theta spectral power in the frontal area during a cold pressor test due to stress and painful activation [27,28]. It was also implicated that heightened delta activity reflects the stress component of human pain responsivity in such a stress task [27]. Thus, the increase in delta and theta activity during the post-stimulus phase for people with a higher stress score may be related to this proposed mechanism. Although no a priori hypothesis was formulated, the interaction effect of the PSS coping factor and the EEG time effect on the alpha band in the frontal region (larger increase during the post-stimulus phase for subjects with a greater coping capacity) may be intuitively logical. Stated otherwise, in subjects with low perceived control, a state of relaxation after a stressor seems to be more difficult to attain, and is in line with results observed in other studies $[4,5,29]$

Since anxiety can be viewed as a form of stress, the results of some studies examining the relationship between anxiety and EEG activity are relevant. A relationship between anxiety and lower alpha activity in the prefrontal cortex is often suggested. The prefrontal cortex is directly connected with the amygdala, hippocampus and hypothalamus. The prefrontal cortex can inhibit limbic activity through these connections, as shown in human anxiety disorder studies [26,30-32]. This mechanism may also apply to the present study, in which the interaction effect between stress coping and spectral power in the pre- and post-stimulus phase was examined. A clear effect was demonstrated in the frontal area for alpha activity, indicating that subjects with a lower coping capacity show a larger decrease in alpha activity. In other words, the impact of the acute stressor appears to be greater in subjects with lower coping capacity.

As to the significant effects observed in the other frequency bands, a clear post hoc explanation is lacking and replication of these findings is needed.

The generalizability of this particular stress experiment to a daily life stress situation may be a point of critique. However, we think that the uncontrollability and unpredictability of the 
stressor in the experimental paradigm are fundamental requirements of a stressful situation in general.

For future research, it would be interesting to use sLORETA analyses to further investigate regulatory mechanisms.

Since multilevel analysis is not frequently used in this area of research, relatively little knowledge on this topic has been gathered. The results of the present article should be interpreted as proof of principle regarding EEG changes during a stress experiment. Since stress reactivity is not a static but a dynamic process, multilevel analysis may be preferred to map out the EEG time course before, during and after a stressor. This statistical technique allows investigators to unravel psychophysiological response mechanisms in stress experiments. The fact that different time effect was found for the anticipatory period and the post-stimulus period does plead for not merely examining main effects within stress experiments, but also investigating the time course. When focussing only on main effects, much of the information on underlying processes may not be revealed. Also, in case of a V-shaped mechanism or an inversed V-shaped mechanism, pre-post effects could be cancelled out and no main effect would be apparent.

In conclusion, this article demonstrates that with the current paradigm stress can be quantified with specific changes in EEG activity. Subsequently, the issue to be investigated is whether this reactivity depends on personal and environmental related variables. In this article the influence of perceived stress on cortical stress reactivity was shown. Including psychosocial variables measuring coping styles as well as stress-related personality aspects allows us to examine the interconnection between mind and body. This may especially be the case in stress research investigating the transformation process of acute stress to chronic stress.

Finally, it is important to mention the potential of this work in the rehabilitation of patients with a stress disorder, such as post-traumatic stress disorder. Since coping capacity is associated with health consequences later in life, it would be interesting to measure the electroencephalographic activity of these patients using the present stress experiment. If EEGactivity related to lower coping capacity could be objectified, early detection and preventive intervention of inadequate coping may be possible.

\section{ACKNOWLEDGMENTS}

We would like to thank Lonneke Bodar and Marga Schnitzeler for their supportive role in this study and Jacco Ronner and Ron Mengelers for technical support.

\section{REFERENCES}

Feuerstein M, Labbé, E. E., \& Kuczmierczyk, A. R. (1986) Health psychology: A psychobiological perspective. New York: Plenum Press.

2. Luijcks R, Hermens HJ, Bodar L, Vossen CJ, Os J, et al. (2014) Experimentally Induced Stress Validated by EMG Activity. PloS one 9: e95215.

3. Lewis RS, Weekes NY, Wang TH (2007) The effect of a naturalistic stressor on frontal EEG asymmetry, stress, and health. Biol Psychol 75: 239-247.

4. Master SL, Amodio DM, Stanton AL, Yee CM, Hilmert CJ, et al. (2009) Neurobiological correlates of coping through emotional approach. Brain Behav Immun 23: 27-35.

5. Goodman RN, Rietschel JC, Lo LC, Costanzo ME, Hatfield BD (2013) Stress, emotion regulation and cognitive performance: the predictive contributions of trait and state relative frontal EEG alpha asymmetry. Int Psychophysiol 87: 115-123.

6. Yu X, Zhang J, Xie D, Wang J, Zhang C (2009) Relationship between scalp potential and autonomic nervous activity during a mental arithmetic task. Auton Neurosci 146: 81-86.

7. Ishitani N, Masumoto Y, Yoshihara T, Yamasaki Y (2005) Changes in electroencephalographic activities following pressure stimulation in humans. Psychiatry Clin Neurosci 59: 644-651.

8. Cipresso P, Gaggioli A, Serino S, Pallavicini F, Raspelli S, et al. (2012) EEG alpha asymmetry in virtual environments for the assessment of stress-related disorders. Stud Health Technol Inform 173: 102-104.

9. Coan JA, Allen JJ (2004) Frontal EEG asymmetry as a moderator and mediator of emotion. Biol Psychol 67: 7-49.

10. Cole C, Zapp DJ, Katherine Nelson S, Perez-Edgar K (2012) Speech presentation cues moderate frontal EEC asymmetry in socially withdrawn young adults. Brain Cogn 78: 156-162

11. Pizzagalli D (2007) Electroencephalography and high-density electrophysiological source localization. In: J. T. Caccioppo LGT, \& G. Berntson, editor. Handbook of psychophysiology (3rd ed). Cambridge, UK: Cambridge University Press. pp. 56-84.

12. Nitschke JB, Heller, W., Miller, G.A. (2000) Anxiety, stress, and cortical brain function. New York: Oxford Universiy Press

13. Oathes DJ, Ray WJ, Yamasaki AS, Borkovec TD, Castonguay LG, et al. (2008) Worry, generalized anxiety disorder, and emotion: evidence from the EEG gamma band. Biol Psychol 79: 165-170

14. Ezzati A, Jiang J, Katz MJ, Sliwinski MJ, Zimmerman ME, et al. (2014) Validation of the Perceived Stress Scale in a community sample of older adults. Int J Geriatr Psychiatry 29: 645-652

15. Vossen H, Van Breukelen G, Hermens H, Van Os J, Lousberg R (2011) More potential in statistical analyses of event-related potentials: a mixed regression approach. International journal of methods in psychiatric research 20: e56-68.

16. Bromm B, Meier W (1984) The intracutaneous stimulus: a new pain model for algesimetric studies. Methods and findings in experimental and clinical pharmacology 6: 405-410.

17. Klem GH, Luders HO, Jasper HH, Elger C (1999) The ten-twenty electrode system of the International Federation. The International Federation of Clinical Neurophysiology. Electroencephalogr Clin Neurophysiol Suppl 52: 3-6.

18. Bellinghausen L, Collange J, Botella M, Emery JL, Albert E (2009) [Factorial validation of the French scale for perceived stress in the workplace]. Sante Publique 21:365-373. 
19. Mitchell AM, Crane PA, Kim Y (2008) Perceived stress in survivors of suicide: psychometric properties of the Perceived Stress Scale. Res Nurs Health 31: 576-585.

20. Nordin M, Nordin S (2013) Psychometric evaluation and normative data of the Swedish version of the 10-item perceived stress scale. Scand J Psychol 54: 502-507.

21. Lazarus RS (1984) Stress, appraisal, and coping / Richard S. Lazarus, Susan Folkman; Folkman S, editor. New York: Springer Pub. Co.

22. Miskovic V, Ashbaugh AR, Santesso DL, McCabe RE, Antony MM, et al. (2010) Frontal brain oscillations and socia anxiety: a cross-frequency spectral analysis during baseline and speech anticipation. Biol Psychol 83: 125-132.

23. Klimesch W (1999) EEG alpha and theta oscillations reflect cognitive and memory performance: a review and analysis. Brain Res Brain Res Rev 29: 169-195.

24. Klimesch W, Schimke H, Schwaiger J (1994) Episodic and semantic memory: an analysis in the EEG theta and alpha band. Electroencephalogr Clin Neurophysiol 91: 428-441.

25. de Quervain DJ, Aerni A, Schelling G, Roozendaal B (2009) Glucocorticoids and the regulation of memory in health and disease. Front Neuroendocrinol 30: 358-370.

26. Roozendaal B, McEwen BS, Chattarji S (2009) Stress, memory and the amygdala. Nat Rev Neurosci 10: 423-433.

27. Chen AC, Dworkin SF, Haug J, Gehrig J (1989) Topographic brain measures of human pain and pain responsivity. Pain 37: 129-141.

28. Chang PF, Arendt-Nielsen L, Chen AC (2002) Dynamic changes and spatial correlation of EEG activities during cold pressor test in man. Brain Res Bull 57: 667-675.

29. Vanneste S, Joos K, Langguth B, To WT, De Ridder D (2014) Neuronal correlates of maladaptive coping: an EEGstudy in tinnitus patients. PLoS One 9: e88253.

30. Pessoa L (2008) On the relationship between emotion and cognition. Nat Rev Neurosci 9: 148-158.

31. de Carvalho MR, Velasques BB, Freire RC, Cagy M, Marques JB, et al. (2013) Alpha absolute power measurement in panic disorder with agoraphobia patients. J Affect Disord 151: 259-264.

32. Groenewegen HJ, Berendse HW, Wolters JG, Lohman AH (1990) The anatomical relationship of the prefrontal cortex with the striatopallidal system, the thalamus and the amygdala: evidence for a parallel organization. Prog Brain Res 85: 95-116: discussion 116-118. 


\section{Chapter 5}

Processing pain: The modifying role of pain hypervigilance on trapezius muscle activity in a pain habituation experiment

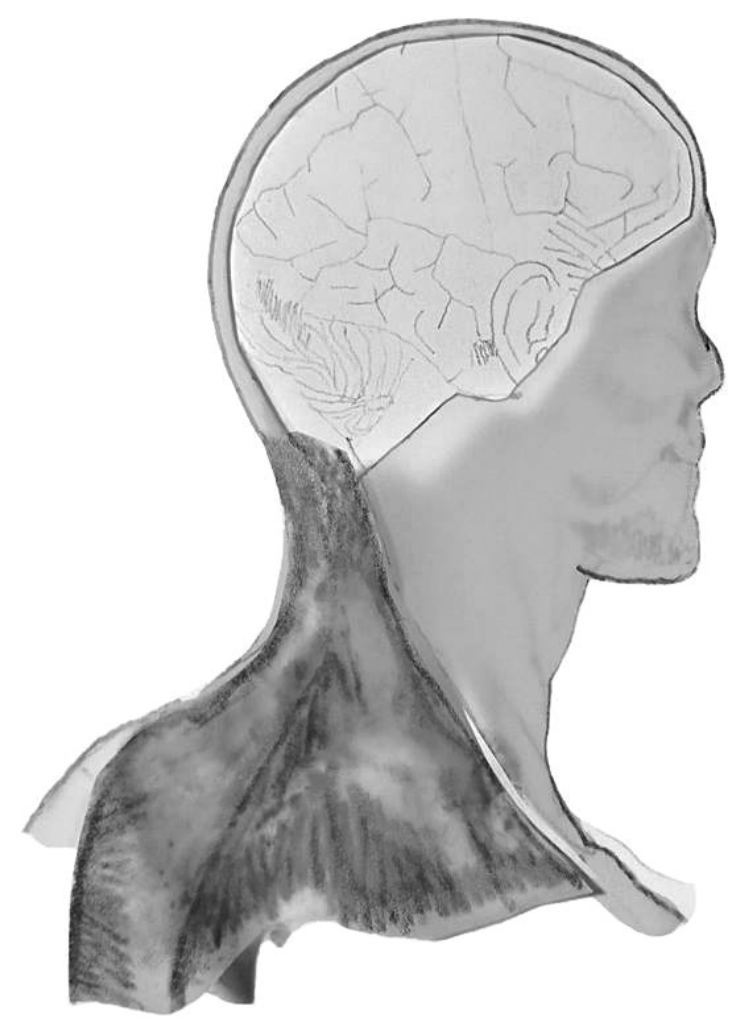

Luijcks R ${ }^{1 *}$, Vossen $\mathrm{CJ}^{1,2}$, Hermens $\mathrm{HJ}^{3,4}$, van Os $\mathrm{J}^{1,5}$, and Lousberg R

1 Department of Psychiatry \& Psychology, Maastricht University Medical Centre, Maastricht The Netherlands 2 Department of Anesthesiology and Pain Medicine, Maastricht University Medical Centre, Maastricht, The Netherlands

3 Roessingh Research and Development, Enschede, The Netherlands

4 Telemedicine Group, Faculty of Electrical Engineering. Mathematics and Computer Science, University of Twente, Enschede, The Netherlands

Department of Psychosis Studies, Institute of Psychiatry, King's College London, King's Health Partners, London, United Kingdom 


\section{ABSTRACT}

Evidence suggests that attention to pain (pain hypervigilance), a key concept in the fearavoidance model, moderates the experience of pain and pain behavior. Here, we examine the moderating effect of pain hypervigilance on EMG (electromyographic) reactivity of the trapezius muscle in a pain habituation experiment.

In a pain-free general population sample $(n=54), E M G$ responses to 25 repeated painful electrical stimuli were assessed. The main aim of the study was to model the time course of EMG activity. Next, it was investigated to what degree pain hypervigilance moderates EMG activity. Pain hypervigilance was indexed using the Pain Vigilance and Attention Questionnaire (PVAQ).

In the anticipatory phase, a linear decrease in EMG activity was observed, followed by an exponential increase. Analyses of the pain reactivity phase showed inverse decreasing EMG activity over time, followed by a linear increase. Higher PVAQ-scores were associated with higher mean level of anticipatory EMG activity, and the linear decrease was reduced to a lesser extent, compared to the low PVAQ-scores.

The influence of pain hypervigilance on linear and non-linear time effects accentuates the importance of a biopsychosocial approach towards pain. Increased EMG activity in anticipation of a painful event, possibly leading to higher pain perception and thus completing a vicious circle, may provide additional evidence for a model of the development of chronic pain

\section{INTRODUCTION}

Pain, as a state in which the integrity of the body is threatened, is a physical stressor. A painful event will not only generate a pain response (reactivity), but can also be accompanied by a state of cognitive stress (anticipatory activity) [1], especially if the upcoming event is uncontrollable and unpredictable [2]. In addition, pain responses are dependent on properties of the upcoming stimulus, such as novelty, intensity and threat value [3].

Previously, we described an experimental stress paradigm, in which a single uncontrollable and unpredictable painful stimulus is presented and stress responses were measured at the level of trapezius muscle electromyographic (EMG) activity [4]. Taking into account the dual nature of the pain stimulus, the EMG time courses during both the anticipatory (stress) phase and the post-stimulus reactivity phase were examined, using multilevel regression analyses. The results demonstrated that anticipatory and reactivity responses were distinctly different, showing a relative increase in EMG activity in the anticipatory phase and a relative decrease in EMG activity during the post-stimulus phase. In a further study (submitted), it was shown that level of childhood adversity modified the anticipatory EMG stress response: a greater level of childhood adversity was associated with an increase in EMG activity during the anticipatory stress phase.

Many studies suggest that attention to pain, a key concept in the fear-avoidance model, influences the experience of pain and pain behavior [5-8]. Pain-related fear can induce a state of preoccupation with or excessive attention to pain, often referred to as hypervigilance $[2,9]$. Pain hypervigilance in turn is likely to influence perceived pain severity and other pain responses [2, 9-13]. In addition, pain hypervigilance maintains and amplifies bodily sensations and thus influences processing and experience of pain [14-16]. The fear-avoidance model provides an important theory for the mechanism in the development of chronic pain. Distraction from pain improves pain tolerance or decreases perception of pain severity.

In order to study these issues in an objective experimental paradigm of physiological pain exposure and physiological pain responses, we investigated the moderating aspect of pain hypervigilance on psychophysiological activity in a pain habituation experiment comprising a series of 25 electrical, painful stimuli [17, 18], executed in a pain-free general population sample. In an experimental design, uncontrollability and unpredictability is reduced. Despite increased level of controllability, the pain habituation experiment nevertheless will contain (a series of) anticipatory (stress) and (pain) reactivity phases. Given the repeated measure design of the experiment, analyses should be controlled for habituation effects [19-21]. 
EMG activity during the anticipatory and pain reactivity phase was modeled, using variables indexing time course. The intermediate phase (i.e. the time between the pain reactivity phase and a subsequent anticipatory phase) was also modeled, though, a priori, no time effects were expected. This basic model was extended by including pain hypervigilance as a potential modifying factor.

It was hypothesized that the anticipatory phase would be characterized by a linear and, based on the findings of our earlier study, independently a non-linear (exponential) increase of EMG activity. Second, an inverse time course, representing a non-linear rapid decline of EMG activity, was expected for the pain reactivity phase. Third, both a linear as well as an inverse habituation effect was expected. Enhanced anticipatory EMG activity was expected for subjects with high scores of pain hypervigilance. Also, a stronger exponential anticipatory effect was predicted. Finally, it was hypothesized that higher attention to pain would be accompanied by a slower return to baseline.

\section{MATERIALS AND METHODS}

\section{Ethics Statement}

The study was approved by the medical ethics committee of the Academic Hospital Maastricht and Maastricht University (NL40284.068.12/METC 12-3-015) and was conducted according to the principles of the Declaration of Helsinki. Subjects provided written informed consent, before the start of the experiment.

\section{Subjects}

The experiment is part of a larger study. Participants consisted of a general population sample residing in the city Maastricht, the Netherlands, and had responded to flyers. Out of a total sample of 121 participants (78 females and 43 males), 54 pain-free (chronic and/or recurrent pain complaints during the past six months as well as acute pain at the moment of inclusion) right-handed subjects were selected (23 males, 31 females). Their age ranged from 18 to 65 years (mean $=37.5$ years; $S D=17.4$ years). Other exclusion criteria were the following: use of antidepressants, antipsychotics, anti-epileptics or anxiolytics in the past year or use of alcoholic beverages in excess of 10 units per day. Subjects were asked to abstain from alcoholic beverages the evening before and caffeine-containing drinks three hours prior to the experiment. Recompense for time spent was 50 euros.

\section{Electro-shocker and stimuli}

An electro-shocker (type Shocko-100-AA-20, developed by Maastricht Instruments BV and approved for usage in experimental studies) was used in order to execute the experiment, comprising 25 electrical stimuli [17]. The shock electrode was applied intracutaneously on the top of the middle finger of the non-dominant left hand, as described by Bromm and Meier [18]. Stimuli were electrical pulses of 10 milliseconds duration. Preparation of the electrode was not experienced as painful.

At the beginning of the procedure, for every subject both the sensation and pain threshold were determined. In order to achieve this, the intensity of the stimulus was gradually increased, starting at zero mA intensity. The sensation threshold was defined as the intensity level of the first stimulus which was consciously experienced. The pain threshold was defined as the intensity level of the first stimulus which was experienced as painful. The procedure of determining these personal thresholds was repeated three times in order to attain reliable estimates. The intensity of the delivered painful stimuli during the experiment was calculated as follows:

\section{Stimulus $=$ pain threshold $+0.25^{*}($ pain threshold - sensation threshold $)$}

This actual intensity level was computed for each subject individually and was used throughout the experiment. As shown in a previous experiment, this intensity level was experienced as being painful, but still acceptable [17]

\section{Procedure}

First, all electrodes were attached, including EMG-, ECG- as well as the shock electrodes. All electrodes were fixed using electroconductive paste. Cardiac activity was recorded with a standard 3-lead ECG. EMG activity was recorded from the left and right upper trapezius muscles (LTM and RTM respectively). Ag/AgCl electrodes were placed on a point $2 \mathrm{~cm}$ lateral to the midpoint between the acromion process and spinous process of the seventh cervical vertebra [22]. A reference electrode was placed over the spinous process of the seventh cervical vertebra. For all recordings, Brainvision BrainAmp Research Amplifier was used. ECG and EMG were sampled with $1000 \mathrm{~Hz}$. All recordings were conducted in a sound-shielded cubicle (7.1 m2).

After determination of the individual pain threshold, subjects were instructed that a sequence of stimuli would follow. No information was given on the exact number of stimuli nor on the intensity level. In order to quantify the subjective pain intensity, subjects were instructed to rate each perceived stimulus on a numerical rating scale (NRS) [23, 24]. As agreed with the subject and for standardization purposes, the first stimulus should be rated as ' 60 ', on a scale of 0 to $100(0=$ no pain, $100=$ strongest pain imaginable). The inter-stimulus intervals (ISI's) were randomized between $9 \mathrm{~s}$ and $11 \mathrm{~s}$ to minimize the likelihood of stimulus predictability 
[25]. Although subjects were told that the stimuli could vary in intensity, all 25 stimuli were of the same intensity level (see above).

Subjects were instructed to keep both hands on the table, palms down, and not to close thei eyes during the measurement period. The whole procedure was controlled by the software program "Presentation 0.71" (Neurobehavioral Systems).

\section{Psychological measurements}

The Dutch version of the Pain Vigilance and Awareness Questionnaire (PVAQ) was used Although this questionnaire was originally developed to investigate vigilance, preoccupation and awareness associated with pain in chronic pain patients, results have shown that it is also a valid and reliable measure in healthy people [10, 26]. The questionnaire consists of 16 items, which can be scored on a six-point scale (minimum score 0; maximum score 80). The questionnaire has a two-factor structure, comprising both attention to pain and attention to changes in pain. For all analyses, a combined sum score of the questionnaire was used.

\section{Offline data processing}

Inspection of the averaged time course of the raw EMG data indicated that the pain reactivity phase was completed within 3 seconds after the stimuli, at which point a return to baseline was reached. Based on this observation, the inter-stimulus interval was divided into three 3-seconds phases: i) the pain reactivity phase (starting immediately after the stimulus), ii) an intermediate phase (immediately following the pain reactivity phase) and iii) the anticipatory phase (starting three seconds before the next stimulus). Given the variable inter-stimulus intervals (ranging from 9 to 11 seconds), a short period of time between the intermediate phase and the next anticipatory phase remains uninvestigated.

For each phase, EMG data was filtered offline (low pass $0.5 \mathrm{~Hz}$, high pass $250 \mathrm{~Hz}$, $50 \mathrm{~Hz}$ notch filter) and segmented into epochs of $250 \mathrm{~ms}$, resulting in 12 consecutive segments. Raw data were visually inspected for artifacts and, if found, excluded from further analyses. The EMG activity of the trapezius muscle was corrected for cardiac activity. Using regression analysis, the variance due to ECG activity was removed from the uncorrected EMG variables and the unstandardized residual was saved. For each $250 \mathrm{~ms}$ epoch, the root mean square value was calculated.

\section{Statistical analysis}

The data had hierarchical structure, each record consisting of a segment (level 1) nested within an individual (level 2), requiring multilevel random regression analyses. EMG activity of the left and right trapezius muscles served as the dependent variable in all models. In order to obtain normality, the dependent variables were log transformed. The predictor variables of primary interest were time-related variables. These variables were elected based on an expected naturalistic behavioral pattern: an increasing pattern in the anticipatory phase, a decreasing pattern in the reactivity phase and a variable pattern in the intermediate phase. Thus, for the anticipatory phase a linear and exponential ( $e^{\text {segment }}$ time effect was modeled. For the pain reactivity phase, in addition to a linear effect, an inverse time effect was added. Additionally, for the intermediate phase, all three time effects were included.

For all three phases, it was tested whether there was a linear or inverse habituation effect over all 25 stimuli. Therefore, stimulus number and its inverse (1/stimulus number) were incorporated in the models.

Next to these time effects within trials (across the 12 consecutive segments) and time effects of habituation (across the 25 stimuli), age, sex, ISI and the calculated difference between the pain threshold and the sensation threshold were added as covariates in all models. Furthermore, in all models random time effects (for both segments and stimuli) as well as a random intercept were estimated.

All statistical analyses were performed using SPSS 22.0. P-values below 0.05 were considered statistically significant.

In order to determine which autoregressive structure should be used, the -2 log likelihood of a so called empty model was calculated. An autoregressive (AR1) structure resulted in significantly better (lowest -2 log likelihood) covariance structure than that of its competitors. The AR1 structure was therefore used for all statistical analyses.

An example of the SPSS syntax of a multilevel model is included in appendix A.

\section{RESULTS}

The sample of pain-free subjects included 54 participants. Four subjects were excluded from the analyses due to technical errors with the shocker or to errors in the measurement of electromyography, leaving $n=50$ analyzable participants (31 females, 19 males). Age ranged from 18 to 65, with a mean age of 36.1 years (SD 16.7). Two subjects did not fill in the PVAQ because of an administrative error. The sum score on PVAQ ranged from 7 to 59 (mean 28.1; $\mathrm{SD}=12.9$ ). The score on the subscale 'attention to pain' ranged from 0 to 35 (mean 14.8; SD 8.0); the score on the subscale 'attention to changes in pain' ranged from 0 to 39 (13.3; SD 7.0). In al 
interaction models, the continuous PVAQ-score was used as interaction term.

The mean level of sensation threshold of painful stimuli was $0.3 \mathrm{~mA}$ (SD $0.2 \mathrm{~mA}$ ); the mean level of the pain threshold was $1.3 \mathrm{~mA}$ (SD $0.75 \mathrm{~mA}$ ); in addition, the mean level of calculated difference between these two levels was $1.0 \mathrm{~mA}$ (SD $0.7 \mathrm{~mA}$ ).

Before presenting the results of the analyses of the three phases separately, it is important to mention the following. With respect to habituation (the time effect across the 25 stimuli), only a linear effect was apparent and was included in all further models. The inverse stimulus component was not used anymore as a predictor variable.

\section{Basic model: linear and exponential EMG time course during the} anticipatory phase

Fig. 1 shows the averaged EMG activity around one stimulus, ranging from 3 seconds before to 6 seconds after the stimulus (including all 3 phases). There is an increase in EMG activity in anticipation of a stimulus, a sharp increase as immediate reaction, followed by a rapid decrease in the post-stimulus phase.

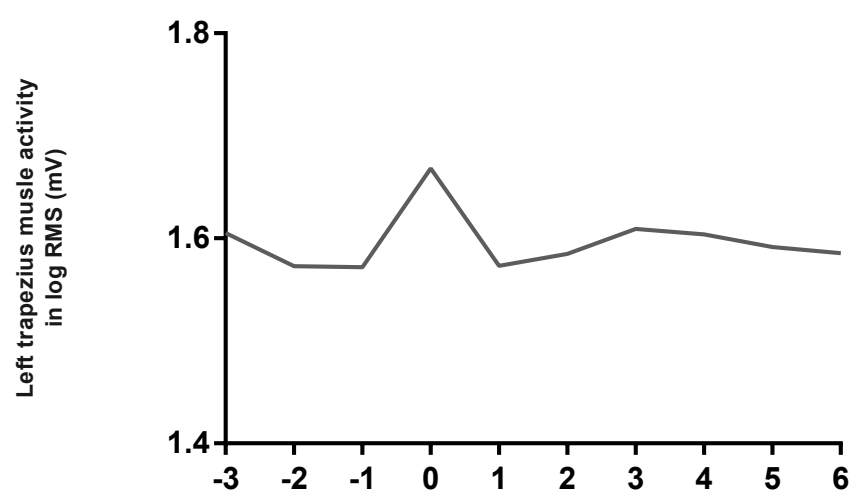

Time (s)

Figure 1. EMG activity during the three phases of the experiment, based on the multilevel models. This figure was computed to illustrate the course of EMG activity. For the anticipatory pre-stimulus phase, a decreasing linear effect ( -3 to -2 seconds pre-stimulus) and a clear exponential effect ( -1 to 0 seconds pre-stimulus) was apparent. For the post-stimulus phase, a rapid decrease in EMG activity ( 0 to 1 second post-stimulus) was found, resulting in a significant inverse time effect. Additionally, a linear increase (1 to 3 seconds post-stimulus) was demonstrated. In the intermediate phase, no significant time effects were demonstrated.
First, it was examined whether there were significant linear and non-linear effects during the anticipatory phase. Analyses showed that both a significant linear and an exponential EMG time course were apparent for both trapezius muscles. The negative T-value of the linear effect indicates a decreasing trend of EMG activity in the pre-stimulus phase. The positive T-value of the exponential effect shows the exponential increase at the end of the anticipatory phase. The corresponding results are depicted in Table 1. It has to be noted that linear time exponential time and stimulus number were included as random components in the model. All random components were highly significant.

No significant interaction effects between the time course within the anticipatory phase (the 12 consecutive segments) and linear habituation over the 25 stimuli were apparent (all p's $>0.464$ ). This applied to both the linear time course as well as the exponential time course.

Table 1. Time effects in the anticipatory pre-stimulus phase.

\begin{tabular}{lcccc}
\hline & \multicolumn{2}{c}{ Left trapezius muscle (LTM) } & \multicolumn{2}{c}{ Right trapezius muscle (RTM) } \\
\hline & T-values & $p$-values & T-values & $p$-values \\
Linear time & -8.496 & $<0.001$ & -7.516 & $<0.001$ \\
Exponential time & 8.035 & $<0.001$ & 7.116 & $<0.001$ \\
\hline
\end{tabular}

Values are T-values and $p$-values for the linear and exponential time effects in the multilevel random regression models. Left trapezius muscle activity and right trapezius muscle activity served as the dependent variables. Main effects of age, sex, inter-stimulus intervals and number of stimulus were included. Incorporated random components of the models were intercept, stimulus number and the exponential time value.

\section{Basic model: linear and inverse EMG time course during the pain reactivity} phase

In the pain reactivity phase (3 seconds after the stimulus), both a linear as well as an inverse time effect were present for the left and right trapezius muscles (all $p<0.001$ ). These results are shown in Table 2.

No significant interaction effect between the linear time course within the reactivity phase (the 12 consecutive segments) and linear habituation over the 25 stimuli was apparent, for neither the left nor the right trapezius muscle (both p>0.698). However, the interaction effect with the inverse time course within the reactivity phase and linear habituation had a T-value of $-3.211(p=0.001)$ for the LTM and a T-value of $-3.834(p<0.001)$ for the RTM. These results indicate a faster return to baseline as the experiment proceeds. The concurrent executed model is included in appendix B. 
Table 2. Time effects in the pain reactivity phase.

\begin{tabular}{lcccc}
\hline & \multicolumn{2}{c}{ Left trapezius muscle (LTM) } & \multicolumn{2}{c}{ Right trapezius muscle (RTM) } \\
\hline & T-values & p-values & T-values & $p$-values \\
Linear time & 5.335 & $<0.001^{* *}$ & 4.226 & $<0.001^{* *}$ \\
Inverse time & 6.845 & $<0.001^{* *}$ & 4.636 & $<0.001^{* *}$ \\
\hline
\end{tabular}

Values are T-values and $p$-values for the linear and inverse time effects in the multilevel random regression models. Left trapezius muscle activity and right trapezius muscle activity served as the dependent variables. Main effects of age, sex, difference between pain threshold and sensation threshold, inter-stimulus intervals and number of stimulus were included. Incorporated random components of the models were intercept, stimulus number and both linear and inverse time effects.

\section{Basic model: linear, inverse and exponential EMG time course during the} intermediate phase

Similar analyses as for the pre- and post-stimulus period were carried out for the intermediate phase. Linear, inverse and exponential functions were examined. Left trapezius muscle activity and right trapezius muscle activity served as the dependent variables. Main effects of age, sex, inter-stimulus intervals and number of stimulus were included. Incorporated random components of the models were intercept, stimulus number and linear, inverse and exponential time variables. Conform a priori expectation, results showed no significant fixed EMG time effects during the intermediate phase for the left and right trapezius muscle, neither for linear time effects ( $p=0.165$ and $p=0.957$, respectively), nor for inverse time effects $(p=0.133$ and $p=0.879$, respectively), nor for exponential time effects ( $p=0.365$ and $p=0.640$, respectively).

\section{Influence of pain hypervigilance on EMG activity during the different} phases of the experiment

In order to examine a possible moderating effect of pain hypervigilance on EMG activity across the different phases, the calculated (continuous) PVAQ-score was added as an interaction variable.

In the anticipatory phase, both an interaction effect of PVAQ-score and linear EMG activity as well as an interaction effect of PVAQ-score and exponential EMG activity were included in the multilevel model. Results showed significant interaction terms for both trapezius muscles (Table 3). The main effect of PVAQ on EMG activity at the beginning of the anticipatory phase appeared to be significant; a higher score is accompanied by higher EMG activity.
Table 3. Interaction between EMG activity and PVAQ-score in the anticipatory phase.

\begin{tabular}{lcccc} 
& \multicolumn{2}{c}{ Left trapezius muscle (LTM) } & \multicolumn{2}{c}{ Right trapezius muscle (RTM) } \\
\cline { 2 - 5 } & T-values & $\mathrm{p}$-values & T-values & $\mathrm{p}$-values \\
\hline Linear time*PVAQ & 2.943 & $0.003^{* *}$ & 2.649 & $0.008^{* *}$ \\
Exponential time*PVAQ & -3.202 & $0.001^{* *}$ & -2.928 & $0.003^{* *}$ \\
\hline
\end{tabular}

Values are T-values and $p$-values for the linear and exponential EMG time courses in the multilevel random regression models. Left trapezius muscle activity and right trapezius muscle activity served as the dependent variables. Main effects of age, sex, inter-stimulus intervals and number of stimulus were included. Incorporated random components of the models were intercept, stimulus number and exponential time variables.

For illustrative purposes, it was decided to depict the EMG time course as moderated by pain hypervigilance. Based on the obtained estimates from the regression models, EMG activity was calculated for both a low (15 points, i.e. mean score - 1 SD in this population) and a high score (41, mean score +1 SD in this population) on the PVAQ. Predicted EMG activity over time during the anticipatory phase for a low and a high PVAQ-score were based on the model estimates (see appendix A) and displayed in Fig. 2.

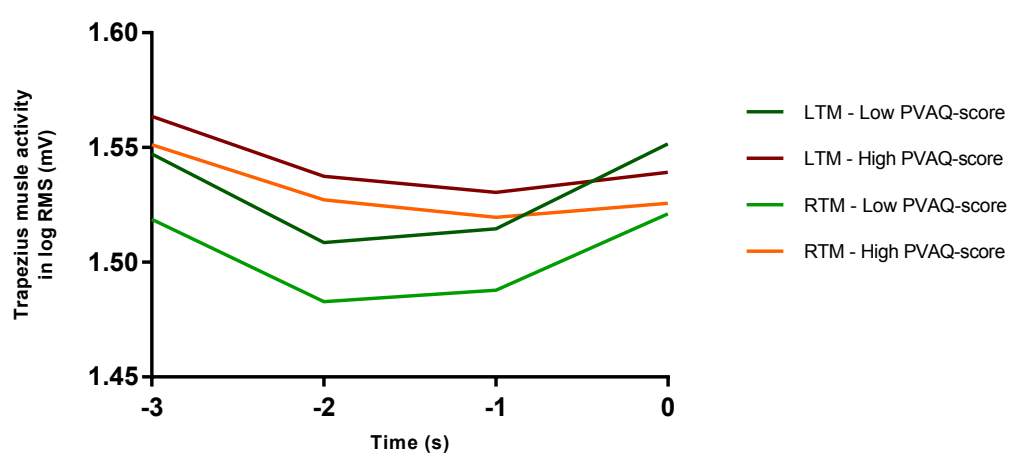

Fig. 2. Fitted left trapezius and right trapezius EMG activity over time during the anticipatory stress period, in interaction with PVAQ-score. For illustrative purposes, EMG activity over time was calculated for a high PVAQ-score (41 points, i.e. mean score + 1 SD in this population) and a low PVAQscore (15 points, i.e. mean score - 1 SD). For subjects with higher pain hypervigilance, higher EMG activity and a slightly decreasing time course can be observed, compared to subjects with a low PVAQ-score, who show lower EMG activity. Additionally, for these subjects, an initial decrease and an exponential time effect at the end of the anticipatory phase can be observed. 
For the pain reactivity phase, both an interaction between the (continuous) PVAQ-score and the linear EMG activity as well as with the inverse EMG activity were included in the multilevel model. Results showed that both interaction terms were not significant, for both trapezius muscles (all p>0.201)

For the intermediate phase, interaction terms between the PVAQ-score and the linear, inverse and exponential time course were included in the multilevel model. Results showed that none of the interaction terms were significant for either trapezius muscles (all $p>0.481$ ).

\section{DISCUSSION}

The first goal of the present study was to examine trapezius muscle activity over time in a pain experiment. Results confirm that the EMG activity in both the anticipatory phase and the pain reactivity phase contained linear and non-linear time effects. These time effects represent the natural course of muscle activity during the experiment.

For the anticipatory phase, a linear reduction in EMG activity and an exponential increase in EMG activity over time were demonstrated (Table 1). The exponential increase indicates that subjects anticipate on painful stimuli, a psychological process which is accompanied by increased muscle activity. Next to this rapid exponential increasing EMG activity just before the stimulus, an independent linear decrease in EMG activity over time was observed. This decreasing EMG activity may be viewed as a late return to baseline in reaction to the previous stimulus.

The analyses on the pain reactivity phase (i.e. the first three seconds post-stimulus) showed an inverse decrease in EMG activity for both trapezius muscles (Table 2). This inverse time course was a priori expected, since it is known that after a painful stimulus the muscle reactivity reaches a maximum relatively fast and then quickly returns towards baseline. After this initial phase of rapidly decreasing EMG activity, a linear increase in EMG activity over time is apparent. These results suggest that the pain reactivity phase has not come to an end yet. However, in the intermediate phase of the experiment (i.e. 3 to 6 seconds post-stimulus), no significant changes in EMG activity could be demonstrated, which indicates that no pain (re)activity is present in this phase anymore.

With respect to a general tendency over all 25 stimuli, a linear habituation of EMG activity was observed in both left and right trapezius muscles, in all phases. This habituation effect was a priori expected and signifies that, on average, the muscle activity of the trapezius muscles reduces as the experiment proceeds.
Concerning the interaction between habituation and EMG activity over time, no effects could be found for the anticipatory and the intermediate phase. In the pain reactivity phase however, an interaction effect between habituation over the 25 stimuli and the inverse EMG activity across the segments was demonstrated. This interaction effect indicates a faster return to baseline as the experiment proceeds, a phenomenon which matches with a natural physiological reaction pattern [27].

For all models, several random effects were demonstrated and turned out to be highly significant, justifying the need for multilevel modelling in psychophysiological research in a repeated measure design. Further, since it was demonstrated in this experiment that the EMG time effects are partly non-linear, only focusing on averaged EMG activity would have been insufficient.

\section{The influence of pain hypervigilance}

The second goal of this study was to investigate whether or not pain hypervigilance has a modifying effect on trapezius muscle activity. To this end, the Pain Vigilance and Attention Questionnaire (PVAQ) was used. During the anticipatory phase, elevated scores on pain vigilance were associated with stable and slightly decreasing EMG activity. On the contrary, low scores on pain vigilance were accompanied by a stronger linear reduction in EMG activity. Additionally, a clear exponential interaction effect was shown at the end of the anticipatory phase. Since a lower score on the PVAQ-questionnaire is associated with lower EMG activity at the beginning of the anticipatory phase, followed by a stronger reduction in EMG activity when compared with high PVAQ-scores, it can be concluded that a low PVAQ-score is associated with a lower mean EMG activity level. This is also illustrated in Fig. 2.

The results suggest that subjects with a relatively low pain hypervigilance-score have lower and more decreasing EMG activity, but show anticipatory reactivity while awaiting the stimuli. n contrast, subjects with a high score maintain more or less stable (slightly decreasing), though elevated EMG activity, without any sign of exponential anticipatory activity. In other words, subjects with high scores of pain vigilance appear to have higher EMG activity in the anticipatory phase, possibly corresponding to a continuous state of attention. Subjects with a low score show increasing EMG activity only at the end of the anticipatory phase, as if intuiting that the stimulus is about to be received. Post hoc, it could be postulated that high PVAQscores are associated with maintenance of high muscle activity in the whole course of the anticipatory phase, and therefore no additional exponential activity at the end of the phase emerges. 
Finally, we examined the role of pain hypervigilance during the pain reactivity phase. It was hypothesized that pain hypervigilance would be associated with a slower return to baseline. However, no significant interaction effects were found. A post hoc interpretation is that this could be due to the fact that the direct reactivity to a stimulus can be viewed as a nociceptive withdrawal reflex $[28,29]$. Cognitive evaluation of the stimulus may not have a moderating effect [30]. Yet, it should be noted that a cognitive evaluation is presumed to exert its influence on the subsequent pre-stimulus phase, reflected by the modifying effect of pain hypervigilance on EMG activity in the following anticipatory phase.

\section{Clinical implications}

To our knowledge, this is the first study to investigate the relationship of pain hypervigilance on trapezius muscle reactivity using a pain habituation experiment in pain-free subjects. In this study, higher scores on pain vigilance were associated with generally heightened anticipatory EMG activity and a different anticipatory exponential effect. In previous studies, it has been hypothesized by several authors that heightened EMG reactivity could play a role in the transition process of acute to chronic pain [31, 32]. Assuming that the results of the present study are generalizable to daily life, the heightened EMG activity in anticipation of a stressor for (relatively) hypervigilant subjects may progress into pain, especially if painful events occur frequently or repetitively. Once the stress-pain liaison is progressing, this could lead to even higher levels of pain vigilance, thus initiating a vicious circle. A biopsychosocial mechanism may thus lead to the development of chronic pain (syndromes). In line with this hypothesis, it is often thought that subjects with chronic pain are characterized by hypervigilance for painrelated information [33], and are vulnerable to maintain a chronic pain state [32].

\section{Limitations}

First, only the muscle activity of the trapezius muscles was measured. The choice for the electrode placement was based on earlier studies, investigating pain and stress. In future studies, it would be interesting to also examine the activity of other muscles (for example the paraspinal muscles) [34, 35]. Second, only pain-free subjects were included in the analyses. In total, a sample of 121 subjects participated. About half of the sample experienced chronic or recurrent pain during the past six months, or was experiencing pain at the moment of inclusion. However, because of the heterogeneity of the 'pain group', differing in both pain state (acute or chronic) as well as in pain location (ranging from head to toe), it was decided not to include these subjects. Nevertheless, it would be fascinating to execute the pain habituation protocol in a specific clinical sample. Finally, the present study was cross-sectional, which does not yield information on causality over time. For future studies, it would be interesting to carry out this experiment in a prospective design.

\section{CONCLUSIONS}

EMG activity in this pain habituation experiment was modeled with multilevel analysis. It was concluded that a distinction should be made between the anticipatory stress of a painful event and the reactivity following this event. Muscle activity of the trapezius muscles was moderated by the level of pain vigilance. This finding underlines the importance of a biopsychosocia approach in the study of pain and stress. The heightened EMG activity in anticipation of a painful event, possibly leading to higher pain perception and thus completing a vicious circle, may provide a foundation of a model for the development of chronic pain. Future studies are required to test this mechanism.

\section{ACKNOWLEDGEMENTS}

We would like to thank Lonneke Bodar and Marga Schnitzeler for their supportive role in this study and Jacco Ronner and Ron Mengelers for technical support. All statistical methods were developed with adequate in house statistical support. 


\section{REFERENCES}

1. Grillon C, Ameli R, Woods SW, Merikangas K, Davis M. Fear-potentiated startle in humans: effects of anticipatory anxiety on the acoustic blink reflex. Psychophysiology. 1991:28(5):588-95. PubMed PMID: 1758934.

2. Crombez G, Van Damme S, Eccleston C. Hypervigilance to pain: an experimental and clinical analysis. Pain. 2005;116(1-2):4-7. doi: 10.1016/j.pain.2005.03.035. PubMed PMID: 15927387.

3. Eccleston C, Crombez G. Pain demands attention: a cognitive-affective model of the interruptive function of pain. Psychol Bull. 1999;125(3):356-66. PubMed PMID: 10349356

4. Luijcks R, Hermens HJ, Bodar L, Vossen CJ, Van Os J, Lousberg R. Experimentally induced stress validated by EMG activity. PLoS One. 2014;9(4):e95215. doi: 10.1371/journal.pone.0095215. PubMed PMID: 24736740; PubMed Central PMCID: PMC3988146.

5. Vlaeyen JW, Linton SJ. Fear-avoidance and its consequences in chronic musculoskeletal pain: a state of the art. Pain. 2000;85(3):317-32. PubMed PMID: 10781906.

6. Crombez G, Eccleston C, Van Damme S, Vlaeyen JW, Karoly P. Fear-avoidance model of chronic pain: the nex generation. Clin J Pain. 2012;28(6):475-83. doi: 10.1097/AJP.0b013e3182385392. PubMed PMID: 22673479.

7. Dittmar $\mathrm{O}$, Baum C, Schneider R, Lautenbacher S. Effects of context and individual predispositions on hypervigilance to pain-cues: an ERP study. J Pain Res. 2015;8:507-21. doi: 10.2147/JPR.S80990. PubMed PMID: 26316802; PubMed Central PMCID: PMC4540212

8. Baum C, Huber C, Schneider R, Lautenbacher S. Prediction of experimental pain sensitivity by attention to painrelated stimuli in healthy individuals. Percept Mot Skills. 2011;112(3):926-46. doi: 10.2466/02.09.22.PMS.112.3.926946. PubMed PMID: 21853779

9. Lautenbacher $S$, Huber C, Kunz M, Parthum A, Weber PG, Griessinger N, et al. Hypervigilance as predictor of postoperative acute pain: its predictive potency compared with experimental pain sensitivity, cortisol reactivity, and affective state. Clin J Pain. 2009:25(2):92-100. PubMed PMID: Medline:19333152.

10. McCracken LM. "Attention" to pain in persons with chronic pain: A behavioral approach. Behav Ther 1997;28(2):271-84. doi: Doi 10.1016/S0005-7894(97)80047-0. PubMed PMID: WOS:A1997XZ53300006.

11. Villemure C, Bushnell MC. Cognitive modulation of pain: how do attention and emotion influence pain processing? Pain. 2002;95(3):195-9. PubMed PMID: Medline:11839418

12. Marker RJ, Stephenson JL, Kluger BM, Curran-Everett D, Maluf KS. Modulation of intracortical inhibition in response to acute psychosocial stress is impaired among individuals with chronic neck pain. J Psychosom Res. 2014;76(3):249-56. doi: 10.1016/j.jpsychores.2013.12.001. PubMed PMID: 24529046.

13. Wong WS, Lam HM, Chow YF, Chen PP, Lim HS, Wong S, et al. The effects of anxiety sensitivity, pain hypervigilance, and pain catastrophizing on quality of life outcomes of patients with chronic pain: a preliminary, cross-section analysis. Qual Life Res. 2014;23(8):2333-41. doi: 10.1007/s11136-014-0683-y. PubMed PMID: 24748558.

14. Hollins M, Harper D, Gallagher S, Owings EW, Lim PF, Miller V, et al. Perceived intensity and unpleasantness of cutaneous and auditory stimuli:an evaluation of the generalized hypervigilance hypothesis. Pain. 2009;141(3):21521. doi: 10.1016/.jpain.2008.10.003. PubMed PMID: 19121558; PubMed Central PMCID: PMC2654196.

15. McDermid AJ, Rollman GB, McCain GA. Generalized hypervigilance in fibromyalgia: evidence of perceptual amplification. Pain. 1996;66(2-3):133-44. PubMed PMID: 8880834

16. Hollins $\mathrm{M}$, Walters $\mathrm{S}$. Experimental hypervigilance changes the intensity/unpleasantness ratio of pressure sensations: evidence for the generalized hypervigilance hypothesis. Exp Brain Res. 2016;234(6):1377-84. do: 10.1007/s00221-015-4541-0. PubMed PMID: 26724932.
17. Vossen H, Van Breukelen G, Hermens H, Van Os J, Lousberg R. More potential in statistical analyses of eventrelated potentials: a mixed regression approach. Int J Methods Psychiatr Res. 2011;20(3):e56-68. doi: 10.1002/ mpr.348. PubMed PMID: 21812066.

18. Bromm B, Meier W. The intracutaneous stimulus: a new pain model for algesimetric studies. Methods Find Exp Clin Pharmacol. 1984;6(7):405-10. PubMed PMID: Medline:6503475.

19. Gold MS, Gebhart GF. Nociceptor sensitization in pain pathogenesis. Nat Med. 2010;16(11):1248-57. doi: 10.1038/ nm.2235. PubMed PMID: 20948530

20. Greffrath W, Baumgartner U, Treede RD. Peripheral and central components of habituation of heat pain perception and evoked potentials in humans. Pain. 2007;132(3):301-11. doi: 10.1016/.jpain.2007.04.026. PubMed PMID: 17533117

21. Hollins $M$, Harper $D$, Maixner W. Changes in pain from a repetitive thermal stimulus: the roles of adaptation and sensitization. Pain. 2011;152(7):1583-90. doi: 10.1016/j.pain.2011.02.049. PubMed PMID: 21454015; PubMed Centra PMCID: PMC3114199.

22. Jensen C, Vasseljen O, Westgaard RH. The influence of electrode position on bipolar surface electromyogram recordings of the upper trapezius muscle. Eur J Appl Physiol Occup Physiol. 1993:67(3):266-73. PubMed PMID. 8223542.

23. Jurth C, Rehberg B, von Dincklage F. Reliability of subjective pain ratings and nociceptive flexion reflex responses as measures of conditioned pain modulation. Pain Res Manag. 2014;19(2):93-6. PubMed PMID: 24555177; PubMed Central PMCID: PMC4028659.

24. Williamson A, Hoggart B. Pain: a review of three commonly used pain rating scales. J Clin Nurs. 2005;14(7):798804. PubMed PMID: Medline:16000093

25. Oka S, Chapman CR, Kim B, Shimizu O, Noma N, Takeichi O, et al. Predictability of painful stimulation modulates subjective and physiological responses. J Pain. 2010;11(3):239-46. doi: 10.1016/j.jpain.2009.07.009. PubMed PMID: 19853519

26. Roelofs J, Peters ML, Muris P, Vlaeyen JW. Dutch version of the Pain Vigilance and Awareness Questionnaire validity and reliability in a pain-free population. Behav Res Ther. 2002;40(9):1081-90. PubMed PMID: 12296493.

27. Rankin CH, Abrams T, Barry RJ, Bhatnagar S, Clayton DF, Colombo J, et al. Habituation revisited: an updated and revised description of the behavioral characteristics of habituation. Neurobiol Learn Mem. 2009;92(2):135-8. do: 10.1016/.nIm.2008.09.012. PubMed PMID: 18854219; PubMed Central PMCID: PMC2754195.

28. Serrao M, Pierelli F, Don R, Ranavolo A, Cacchio A, Curra A, et al. Kinematic and electromyographic study of the nociceptive withdrawal reflex in the upper limbs during rest and movement. J Neurosci. 2006;26(13):3505-13. doi: 10.1523/NNEUROSCI.5160-05.2006. PubMed PMID: 16571758.

29. Peterson CL, Riley ZA, Krepkovich ET, Murray WM, Perreault EJ. Withdrawal reflexes in the upper limb adapt to arm posture and stimulus location. Muscle Nerve. 2014;49(5):716-23. doi: 10.1002/mus.23987. PubMed PMID: 23929755

30. Terkelsen AJ, Andersen OK, Molgaard H, Hansen J, Jensen TS. Mental stress inhibits pain perception and heart rate variability but not a nociceptive withdrawal reflex. Acta Physiol Scand. 2004:180(4):405-14. PubMed PMID: Medline:15030382.

31. Heydari A, Nargol AVF, Jones APC, Humphrey AR, Greenough CG. EMG analysis of lumbar paraspinal muscles as a predictor of the risk of low-back pain. Eur Spine J. 2010;19(7):1145-52. PubMed PMID: Medline:20127260

32. Burns JW. The role of attentional strategies in moderating links between acute pain induction and subsequent psychological stress: evidence for symptom-specific reactivity among patients with chronic pain versus healthy nonpatients. Emotion. 2006:6(2):180-92. doi: 10.1037/1528-3542.6.2.180. PubMed PMID: 16768551. 
33. Pincus T, Morley S. Cognitive-processing bias in chronic pain: a review and integration. Psychol Bull. 2001;127(5):599-617. PubMed PMID: 11548969.

34. Lewis S, Holmes P, Woby S, Hindle J, Fowler N. The relationships between measures of stature recovery, muscle activity and psychological factors in patients with chronic low back pain. Man Ther. 2012;17(1):27-33. do 10.1016/j.math.2011.08.001. PubMed PMID: 21903445.

35. Wolff B, Burns JW, Quartana PJ, Lofland K, BruehI S, Chung OY. Pain catastrophizing, physiological indexes, and chronic pain severity: tests of mediation and moderation models. J Behav Med. 2008;31(2):105-14. doi: 10.1007/ s10865-007-9138-z. PubMed PMID: 18158618; PubMed Central PMCID: PMC4180106.

\section{Appendix A}

MIXED Log_RMS_left WITH age sex ISI stimulus linear_time exponential_time PVAQ_score difference_pain_sensation_threshold

/CRITERIA $=$ CIN(95) MXITER(100) MXSTEP(10) SCORING(1) SINGULAR(0.000000000001) HCONVERGE(0,

ABSOLUTE) LCONVERGE(0, ABSOLUTE) PCONVERGE(0.000001, ABSOLUTE)

$/ F \mid X E D=$ age sex $|S|$ stimulus linear_time exponential_time PVAQ_score

difference_pain_sensation_threshold

linear_time* PVAQ_score exponential_time* PVAQ_score |SSTYPE(3)

METHOD=REML

/PRINT=SOLUTION TESTCOV

/RANDOM=INTERCEPT exponential_time stimulus | SUBJECT(ppn) COVTYPE(UN)

/REPEATED = time_within_subject | SUBJECT(ppn) COVTYPE(AR1). 


\section{Appendix B}

Estimates of Fixed Effectsa

\begin{tabular}{|c|c|c|c|c|c|c|c|}
\hline \multirow{2}{*}{ Parameter } & \multirow{2}{*}{ Estimate } & \multirow{2}{*}{ Std. Error } & \multirow{2}{*}{ df } & \multirow{2}{*}{$\mathrm{t}$} & \multirow{2}{*}{ Sig. } & \multicolumn{2}{|c|}{$95 \%$ Confidence Interval } \\
\hline & & & & & & Lower Bound & Upper Bound \\
\hline Intercept & 1,382239 & 140553 & 47,925 & 9,834 &, 000 & 1,099627 & 1,664851 \\
\hline $\begin{array}{l}\text { Difference_pain_ } \\
\text { sensation_threshold }\end{array}$ & ,001273 & ,002930 & 40,959 &, 435 &, 666 &,- 004644 & ,007191 \\
\hline Age &,- 002764 & 002451 & 40,960 & $-1,128$ & ,266 & -007714 &, 002185 \\
\hline Sex & 001113 & ,086720 & 40,960 &, 013 & 990 &,- 174026 & , 176252 \\
\hline$|S|$ & 009779 & 002960 & 3850,798 & 3,304 & ,001 & ,003976 & ,015583 \\
\hline Stimulus number &,- 005504 &, 001871 & 44,685 & $-2,942$ &, 005 &,- 009274 &,- 001735 \\
\hline Segment & 005368 & 001278 & 43,987 & 4,201 & ,000 & 002792 & 007943 \\
\hline Segment_invers & , 124706 & ,021969 & 56,894 & 5,676 &, 000 & ,080712 & , 168699 \\
\hline $\begin{array}{l}\text { Stimulus number } \\
* \text { Segment_invers }\end{array}$ &,- 002270 & ,000592 & 12977,286 & $-3,834$ & ,000 & -003431 &,- 001109 \\
\hline
\end{tabular}

a. Dependent Variable: Log_RMS_rechts. 


\section{Chapter 6}

Summary, discussion and conclusions

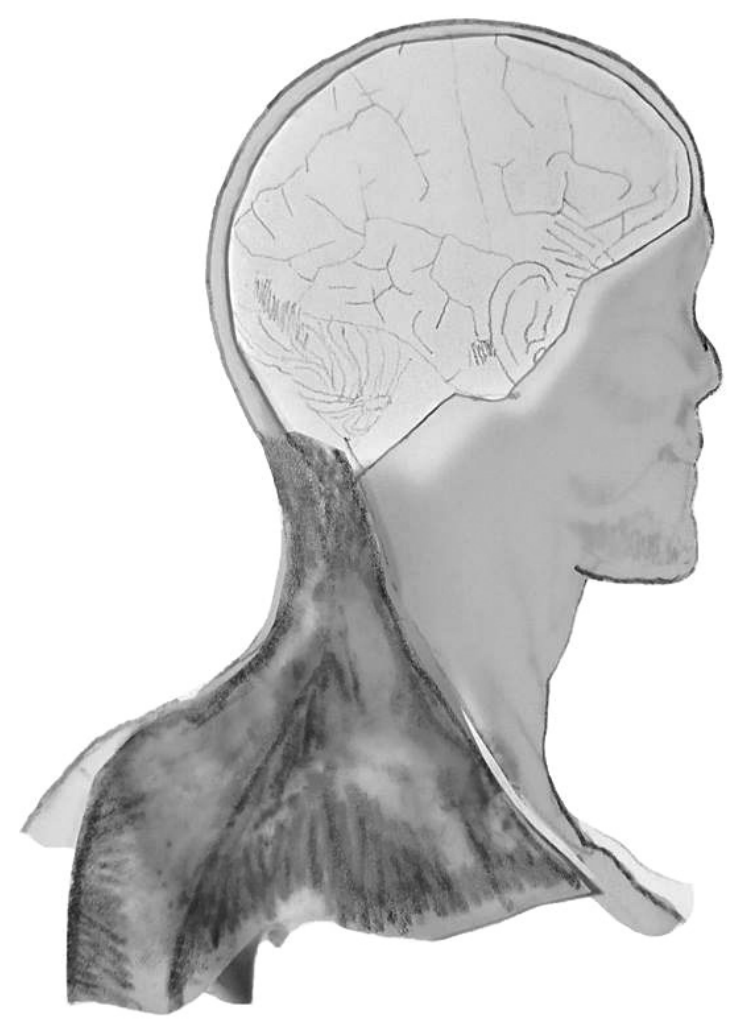


The purpose of this thesis was to investigate psychophysiological reactions in relation to stress and pain, to attain greater insight into the underlying mechanisms and to determine factors associated with, or having influence on, the subjects' vulnerability to develop stress-related health problems or (chronic) pain.

To unravel some of the underlying mechanisms of stress and pain, examining both central and peripheral psychophysiological correlates, the data of the studies presented in this thesis were analyzed with multilevel random regression analyses [1]. More specifically, the focus was to study pain- and stress-related electroencephalographic (EEG) and electromyographic (EMG) activity in two experimental designs. First, we developed a novel stress experiment [2, 3]. Both

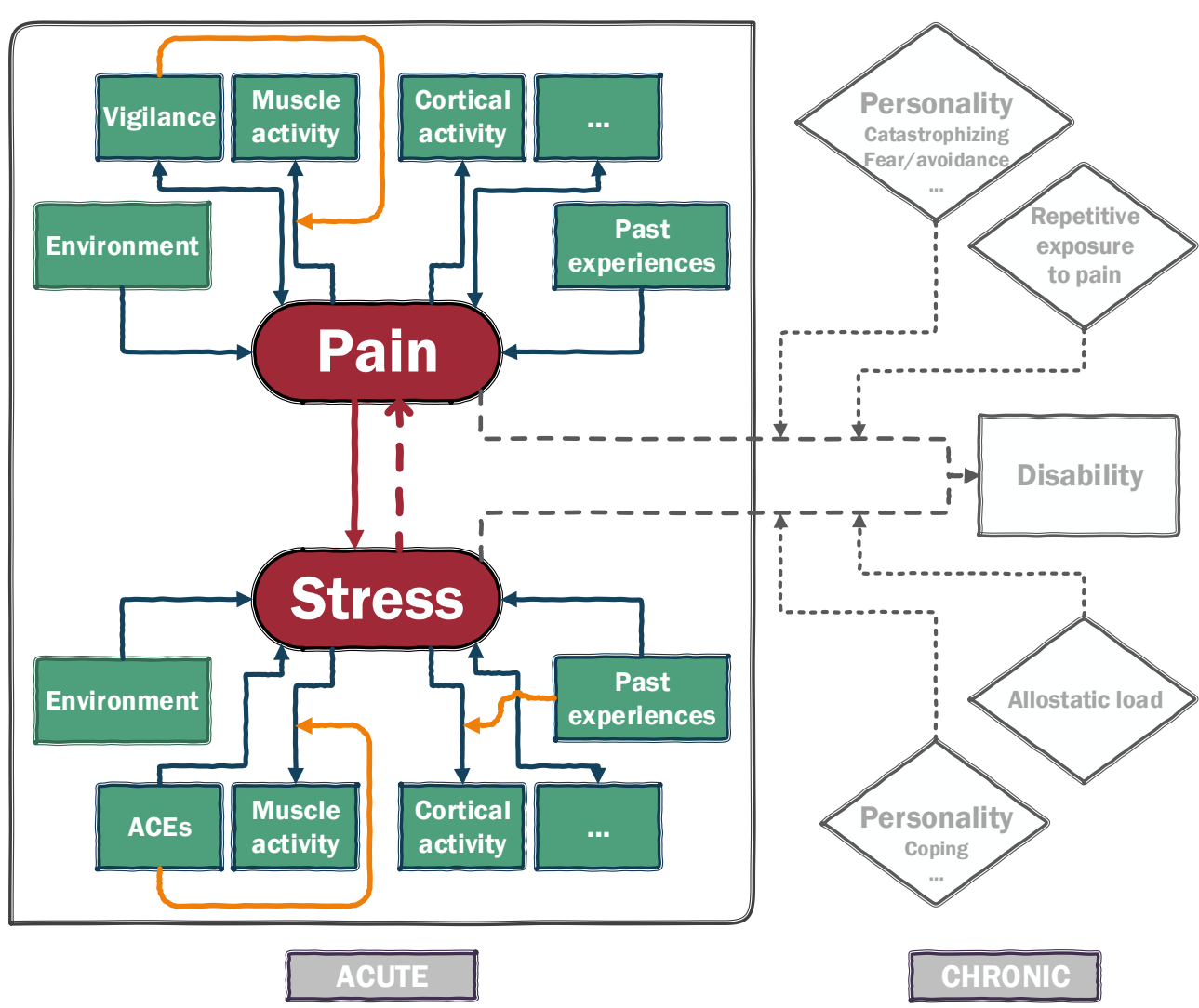

Figure 1. Adapted biopsychosocial model of stress and pain, showing the interplay between the experience of stress and pain. Pain and stress are constructs, which are influenced by several factors. The three orange arrows display three key interactions between psychophysiological activity and psychological behavior, as presented in this thesis. muscle activity and cortical activity were measured and examined. Additionally, in order to obtain further insight into the biopsychosocial mechanisms underlying stress, the influence of several psychological factors on psychophysiological reactivity was investigated.

In addition to this stress experiment, a pain habituation experiment was conducted in order to map the psychophysiological reactivity in relation to pain. Furthermore, the influence of pain hypervigilance on muscle reactivity in this pain habituation experiment was investigated. As evident from the literature, pain and stress can be best studied within a biopsychosocial framework [4-6]. Based on the tight relationship between pain and stress, an example of such a model was proposed in the introduction section of this thesis. An adapted version of this proposed model is shown below. It now includes three additional effects based on the findings of the studies included in this thesis. The three orange arrows display the three key interactions between psychophysiological activity and psychological behavior, as presented in this thesis.

\section{STRESS REACTIVITY AND THE TRAPEZIUS MUSCLES: IS THERE ROOM} FOR 'PSYCH'IN PSYCHOPHYSIOLOGY?

The first aim of this thesis focused on the reactivity of the trapezius muscles within a stres experiment. In Chapter 2, the newly designed stress paradigm was presented. Stimuluselectrodes were placed on the subject's middle finger and one single uncontrollable and unpredictable nociceptive stimulus was presented. Subjects were unaware of the precise moment of stimulus delivery and its intensity level. In this way, a dual stress induction was introduced, consisting of the announcement of the impending receipt of a single electric shock, uncontrollable and unpredictable, thus inducing a cognitive anticipatory stress phase followed by a nociceptive stimulus. EMG activity was measured during this experiment. The differences in mean EMG activity levels in the three phases of the experiment (baseline, pre-stimulus period and post-stimulus period) were in accordance with the intended stress induction: mean EMG activity level was higher in the pre-stimulus period compared with both the baseline and the post-stimulus phase. The second purpose of this study was to introduce multilevel random regression modeling, a technique which was used throughout all the succeeding studies presented in this thesis. Linear and non-linear effects were demonstrated. In conclusion, the results of the first study confirm that the newly-developed stress paradigm induces cognitive stress, resulting in altered muscle activity. It has been clearly demonstrated that the change in muscle activity over time emerges in both linear and non-linear courses and is influenced by the level of subjective stress of the participants. 
In Chapter 3, EMG stress reactivity was investigated as a physiological marker in relation to early life adversity (also called adverse childhood experiences; both terms are used interchangeably in this thesis). In this study, only the anticipatory phase of the stress experiment was used. The sample size of the study was extended. Although several adult stress-related outcomes are moderated by adverse childhood experiences (ACEs), no previous studies had reported on the influence of ACEs on EMG reactivity as a psychophysiological stress measure. Results showed an association between ACEs and altered EMG reactivity, particularly for ACEs in early childhood (0-11 years). No inter-visit ( $t=0$ and $t=6$ months) difference was observed, suggesting a reliable influence of ACEs on psychophysiological activity. A higher ACE-score was accompanied by increased muscle activity in both trapezius muscles during the anticipatory stress phase. Adverse childhood experiences during early childhood were associated with increased EMG stress reactivity, for both trapezius muscles. For ACEs during adolescence (12-17 years) this was less prominent and apparent only for the left trapezius muscle.

\section{MULTILEVEL MODELING AND PSYCHOPHYSIOLOGICAL OUTCOMES}

From Chapters 2 and 3, the following conclusions can be drawn. The validity of the stress experiment was demonstrated: subjective stress was reported, and as a priori expected, trapezius muscle activity was influenced by both stressors (anticipatory stress and physical stress caused by the stimulus). In concordance with these results, several studies observed a difference in EMG activity pre- and post-stimulus [7-9]. In contrast to these studies, we were also able to demonstrate non-linear effects by using multilevel random regression analyses. Multilevel models recognize the existence of a hierarchy in a dataset, by allowing for residual components at each level in the hierarchy [10]. Moreover, this statistical technique allows the investigation of both linear and non-linear time courses. Both types of time course were expected, since physiological activity of the body is a natural process. Next, random intercepts can be modeled, thus allowing correction for inter-individual differences [10]. Datasets with repeated measures, such as those used in all the studies presented in this thesis, are suitable for using multilevel analyses, since repeated measures are nested within subjects,

Matters really starts to get interesting when this statistical technique is applied to facilitate the examination of the interaction between psychological measurements and change in psychophysiological activity over time. In the first study, it is important to note that there was no association between the level of subjective anticipatory stress and the mean EMG activity level. It was found, however, that the non-linear time courses in EMG activity, both quadratic and inverse, were moderated by the anticipatory stress level. The discrepancy between these two findings illustrates the importance of using multilevel modeling in this kind of experiments, since it provides us with additional information that might remain hidden without using this technique. All the above findings emphasize the importance of employing advanced statistical techniques to examine natural processes.

\section{THE EFFECT OF CHILDHOOD ADVERSITY}

In Chapter 3, an interaction effect with early life adversity was examined. It was interesting to see that an association between ACEs and altered EMG reactivity could be demonstrated particularly for ACEs in early childhood. This is in line with previous research [11, 12] showing more enhanced effects on adult mental health outcomes associated with exposure to early life adversity at a younger age. The fact that adverse childhood events influence stress reactivity of the trapezius muscle later in life might contribute valuable information about the relationship between early life adversities and the development of several stress-related musculoskeletal disorders. The finding that there was no inter-visit difference in EMG reactivity between the first and second visit (six months between both visits), suggests that this EMG reactivity can be viewed as a robust characteristic. This finding fortifies the thought that altered muscle reactivity due to adverse childhood experiences is of persistent influence. It can be therefore reasoned that when this altered muscle activity could lead to a stress disorder, it would be possible to intervene. In this way, it would be possible to use, for example, myofeedback of the trapezius muscles, in order to create awareness of heightened muscle activity and to adjust this activity (e.g. see Dellve et al. and Larsman et al. [13, 14]). Furthermore, with the knowledge that several other psychophysiological measures are influenced by childhood abuse, therapeutic interventions, focusing on the prevention of the development of stress disorders later in life, should be considered. Recording trapezius muscle activity during a stress experiment could provide us with information about the initial baseline stress reactivity and later progress.

\section{STRESS REACTIVITY AND THE BRAIN}

Just as muscle activity can be viewed as a peripheral correlate of stress, cortical activity can be studied as a central correlate. In the study described in Chapter 4, we analyzed the cortical activity, during the previously mentioned stress experiment, using both the pre-stimulus phase as well as the post-stimulus phase. Again, multilevel modeling was used, allowing us to investigate the ongoing EEG activity over time. As for the EEG time course of alpha activity pre- and post-stimulus, it was demonstrated that this activity increases more during the poststimulus period than during the pre-stimulus period, as a priori expected. Furthermore, a larger decreasing effect of gamma activity in the post-stimulus period was observed, when compared with the pre-stimulus phase. These effects were mostly found in the frontal, temporal and central regions. In addition to the a priori hypothesized effects, an increasing effect in the slow beta band was detected. Alongside these main effects, the Perceived Stress 
Scale (PSS-10) was used to investigate whether the EEG time course depends on perceived stress and coping capacity. Significant interaction effects between the PSS-10 subscales and the EEG changes over time were demonstrated. Interestingly, the interaction effects between coping and stress subscales with the EEG time course differed. Concerning the stress factor of the PSS-10, a higher perceived stress score was accompanied by a greater increase in delta and theta activity during the post-stimulus phase.

Contrary to this finding, the analyses of the coping factor of the PSS-10 demonstrated that low coping capacity is accompanied by a greater decrease in slow beta, fast beta and gamma activity during the post-stimulus phase. Significant effects on frontal alpha activity were also observed.

With the development of advanced techniques such as Functional magnetic resonance imaging (fMRI) in the world of brain research, it sometimes seems as if electroencephalography has entered oblivion. However, the value of EEG should not be underestimated. Electroencephalography is a relatively cheap, non-invasive technique, with a high tempora resolution, which provides us with a wealth of information. The results described in the proofof-principle-study in Chapter 4 show that more information can be derived from EEG than is usually assumed.

In the literature, alpha activity was related to stress multiple times [15, 16]. The results in Chapter 4, however, not only confirmed this relationship, but also indicated a direction of the effect. More specifically, the results allowed us to model a naturalistic course over time, approaching the authentic functioning of the brain. We encourage researchers to consider this technique, and even combine it with additional image forming.

It is interesting to note that the influence of psychological factors can be objectified. Both factors of the PSS (coping and distress subscales) have a different influence. Although the factors are derived from the same questionnaire, it is reasonable to assume that both factors are related to different pathways in the brain [17, 18]. The challenge in this kind of research is to explain the results and link them to the different areas of the brain. As suggested before, it might be interesting to use fMRI in addition, to provide valuable information about underlying sources [19-22].

\section{PAIN AND HABITUATION}

Finally, in Chapter 5, we examined trapezius muscle activity over time in a pain habituation experiment using multilevel modeling analysis. The pain habituation experiment comprises a series of 25 repeated painful electrical stimuli on the left middle finger. The EMG trapezius muscle activity of the anticipatory and pain reactivity phase (three seconds before and after every painful stimulus respectively) was assessed in a pain-free population. Additionally, it was investigated whether an overall habituation effect was present during the experiment Furthermore, the modifying effect of hypervigilance on trapezius muscle activity was demonstrated. For the anticipatory phase, a linear reduction in EMG activity and an exponential increase in EMG activity over time were demonstrated. The analyses of the pain reactivity phase (i.e. the first three seconds post-stimulus) showed an inverse decrease in EMG activity for both trapezius muscles, followed by a linear increase in EMG activity over time. With respect to a general tendency over all 25 stimuli, a linear habituation of EMG activity was observed in both left and right trapezius muscles. Additionally, in the pain reactivity phase an interaction effect between habituation over the 25 stimuli and the inverse EMG activity across the segments was demonstrated. This interaction effect indicates a faster return to baseline as the experiment proceeds, a phenomenon which matches a natural physiological reaction pattern. As to the influence of hypervigilance, subjects with high scores of pain vigilance appear to have higher EMG activity in the anticipatory phase, possibly corresponding to a continuous state of attention [23]

Habituation can be viewed as a basic mechanism, enabling people to adapt to repeated exposure to painful or stressful events [24]. Several studies have demonstrated the complexity of habituation mechanisms. In order to gather as much mechanistic knowledge as possible, more studies on habituation are strongly recommended [25-29]; especially on the interrelationship between psychological factors and physiological habituation [30, 31]. With the study in Chapter 5, we tried to model an interaction between physiology and psychological behavior during an experiment in which a series of painful stimuli was presented. The results described in Chapter 5 suggest a relationship between physiological habituation and psychological factors. In future studies a three-way-interaction, being habituation over a series of stimuli $X$ pain reactivity $\mathrm{X}$ hypervigilance, would be very interesting to explore.

The results of Chapter 5 may contribute to a theoretical modeling of habituation and emphasize the need for a multidimensional assessment of stress- and pain-related problems. Besides, the results can provide an impetus to develop new or alternative therapeutic interventions. Therapy might focus on myofeedback and/or the modification of the set of pain- and stressrelated cognitions.

\section{LIMITATIONS AND SUGGESTIONS FOR IMPROVEMENTS}

The studies presented in this thesis have some limitations, which have already been discussed 
in each separate chapter. A few general limitations of the research presented here should nonetheless be pointed out. For the EMG-related studies, only the muscle activity of the trapezius muscles was measured. Collecting EMG measurements at multiple locations on the body could be considered, since EMG activity of the trapezius muscles in stressful situations embeds other information than, for example, the cervical muscles, the paraspinal muscles and the facial muscles [32-35]. For future research it is recommended that there should be further study of muscle activity at these places.

Furthermore, it is important to note that the statistical techniques used in this study do not allow us to examine an unlimited number of segments of the measured EMG activity Therefore, it was necessary to find a balance between the number of segments and the length of the total phase about to be analyzed. In the case of very short segments (e.g. less than 250 $\mathrm{ms})$, the number of segments would be very high, making it impossible, at least with current software and hardware, to analyze the entire length of the total phase (pre- or post-stimulus).

Along with the suggested alterations of EMG measurements, researchers may opt to include information on cortisol levels in the future in order to further validate the impact of the pain and stress experiment on subjects. Finally, in order to augment the credibility and validity of certain findings, the inclusion of additional questionnaires, such as the Childhood Trauma Questionnaire, could be considered [36, 37]

\section{BACK TO THE FUTURE}

Within the findings of this thesis, the importance of replicability, as it applies to all research should be noted. A number of topics also remain unexamined. For future research, a range of research questions can be proposed. In the next section, some of these questions will be addressed.

\section{Clinical populations}

To enlarge the clinical applicability of the current results demonstrated throughout this research, additional research within specific clinical populations is required in order to create a larger contrast between groups. In the present thesis, the modulatory effects of stress, pain and childhood adversity on psychophysiological measures have been investigated. To continue along this path, future research should focus on stress-related diseases, such as posttraumatic stress syndromes, chronic pain patients and a clinical population of subjects who suffered childhood abuse.
In Chapter 5 of this thesis, a pain-free population was used. Although a large part of the study population reported either acute or chronic pain, a broad dissemination of different complaints was observed. The optimal research group might be a group consisting of subjects suffering from neck and shoulder pain. It would be interesting to examine the contrast between subjects suffering from acute pain and healthy controls on one side, and subjects suffering from chronic pain ("persistent or recurrent pain lasting longer than 3 months" [38]) and healthy controls on the other side.

\section{Interventional options}

In the light of therapeutic options, it would be interesting to intervene experimentally in the clinical groups mentioned immediately above. For example, a clinical population of subjects with chronic pain could be randomized into two groups, starting by executing the protocol and consecutively using myofeedback as a therapeutic intervention. This study could even be executed double-blind, using sham-myofeedback in the control group. After six months, the effect of the intervention could be objectified by re-administering the questionnaires and by executing the experimental protocol again.

\section{Subgroups}

Another tactic could be to focus more on specific subgroups within the clinical populations mentioned above. For example, in Chapter 5, an interaction between pain hypervigilance and psychophysiological pain reactivity was demonstrated. The paradigm mentioned above, comparing a group of subjects experiencing acute pain with a group of healthy controls, could be extended by examining pain hypervigilance. As such, the psychophysiological activity of four groups could be compared: healthy controls, whether hypervigilant for pain or not, and subjects who experience acute pain, whether hypervigilant for pain or not. These analyses would allow us to distill the underlying mechanisms and the impact of pain hypervigilance in both groups.

Similarly, it would be challenging to distinguish two subgroups within a group of subjects who underwent childhood abuse in the past: one being a group of subjects who do not report any complaints related to this abuse any more, the other a group of subjects who still experience negative consequences as a result of abuse in the past. In order to better understand a possible contrast between the two clinical subgroups, a third healthy control group might be added.

\section{Prospective studies}

Alongside these cross-sectional study designs, another prospective study design would allow us to investigate the development of certain phenomena and provide additional information. 
When examining a study population with subjects suffering from acute pain in a prospective design, including interaction with the degree of the subjects' pain hypervigilance, the evolvement of acute pain into chronic pain could be objectified and the link between these mechanisms and psychophysiological activity could possibly be explained.

\section{FINAL REMARKS}

The main purpose of this thesis was to examine psychophysiological activity in stress and pain as ongoing processes and their interaction with psychological behavior. The results have shown that early life adversity, perceived stress and hypervigilance all influence the psychophysiological activity provoked by pain and stress in an experimental setting. These results contribute to a better understanding of the underlying mechanisms of the complexity of pain and stress. Concerning therapeutic options, the results of this thesis reinforce the idea that the complaints of patients suffering from stress or pain should be placed in a biopsychosocial context in order that they may be optimally treated.

\section{REFERENCES}

1. Myers, N.D., et al., Multilevel modeling in psychosomatic medicine research. Psychosom Med, 2012. 74(9): p. 925-36.

2. Luijcks, R., et al., Experimentally induced stress validated by EMG activity. PLoS One, 2014. 9(4): p. e95215.

3. Luijcks, R., et al., The Influence of Perceived Stress on Cortical Reactivity: A Proof-Of-Principle Study. PLoS One, 2015. 10(6): p. e0129220.

4. Contrada, R.J. and A. Baum, eds. The Handbook of Stress Science: Biology, Psychology, and Health. 2010, Springe Publishing Company

5. Gatchel, R.J., et al., The biopsychosocial approach to chronic pain: scientific advances and future directions. Psychol Bull, 2007. 133(4): p. 581-624.

6. Turk, D.C. and A. Okifuji, Psychological factors in chronic pain: evolution and revolution. I Consult Clin Psychol, 2002. 70(3): p. 678-90.

7. Roman-Liu, D., et al., The influence of mental load on muscle tension. Ergonomics, 2013. 56(7): p. 1125-33.

8. Shahidi, B., A. Haight, and K. Maluf, Differential effects of mental concentration and acute psychosocial stress on cervical muscle activity and posture. J Electromyogr Kinesiol, 2013. 23(5): p. 1082-9.

9. Westgaard, R.H., Effects of physical and mental stressors on muscle pain. Scand J Work Environ Health, 1999. 25 Suppl 4: p. 19-24.

10. Hox, J.J., J.K. Roberts, and M. European Association of, Handbook of Advanced Multilevel Analysis. 2011: Routledge

11. Bunce, S.C., R.J. Larsen, and C. Peterson, Life after trauma: personality and daily life experiences of traumatized people. J Pers, 1995. 63(2): p. 165-88.

12. Glaser, J.P., et al., Childhood trauma and emotional reactivity to daily life stress in adult frequent attenders of general practitioners. J Psychosom Res, 2006. 61(2): p. 229-36.

13. Dellve, L., et al., Myofeedback training and intensive muscular strength training to decrease pain and improve work ability among female workers on long-term sick leave with neck pain: a randomized controlled trial. Int Arch Occup Environ Health, 2011. 84(3): p. 335-46.

14. Larsman, P., et al., Prognostic factors for the effect of a myofeedback-based teletreatment service. J Telemed Telecare, 2010. 16(6): p. 336-43.

15. Goodman, R.N., et al., Stress, emotion regulation and cognitive performance: the predictive contributions of trait and state relative frontal EEG alpha asymmetry. Int J Psychophysiol, 2013. 87(2): p. 115-23.

6. Lewis, R.S., N.Y. Weekes, and T.H. Wang, The effect of a naturalistic stressor on frontal EEG asymmetry, stress, and health. Biol Psychol, 2007. 75(3): p. 239-47.

17. Keay, K.A. and R. Bandler, Parallel circuits mediating distinct emotional coping reactions to different types of stress. Neurosci Biobehav Rev, 2001. 25(7-8): p. 669-78.

18. Master, S.L., et al., Neurobiological correlates of coping through emotional approach. Brain, Behavior, and Immunity, 2009. 23(1): p. 27-35.

19. Emmert, K., et al., Active pain coping is associated with the response in real-time fMRI neurofeedback during pain. Brain Imaging and Behavior, 2016: p. 1-10. 
20. Stoeter, P., et al., Cerebral activation in patients with somatoform pain disorder exposed to pain and stress: an fMR study. Neuroimage, 2007. 36(2): p. 418-30.

21. Garfinkel, S.N., et al., Impaired contextual modulation of memories in PTSD: an fMRI and psychophysiological study of extinction retention and fear renewal. J Neurosci, 2014. 34(40): p. 13435-43.

22. Boecker, R., et al., Impact of early life adversity on reward processing in young adults: EEG-fMRI results from a prospective study over 25 years. PLoS One, 2014. 9(8): p. e104185.

23. Eccleston, C. and G. Crombez, Pain demands attention: a cognitive-affective model of the interruptive function of pain. Psychol Bull, 1999. 125(3): p. 356-66.

24. Rankin, C.H., et al., Habituation revisited: an updated and revised description of the behavioral characteristics of habituation. Neurobiol Learn Mem, 2009. 92(2): p. 135-8,

25. Grissom, N. and S. Bhatnagar, Habituation to repeated stress: Get used to it. Neurobiology of Learning and Memory, 2009. 92(2): p. 215-224

26. Schommer, N.C., D.H. Hellhammer, and C. Kirschbaum, Dissociation between reactivity of the hypothalamuspituitary-adrenal axis and the sympathetic-adrenal-medullary system to repeated psychosocial stress. Psychosom Med, 2003. 65(3): p. 450-60.

27. Wust, S., et al., Habituation of cortisol responses to repeated psychosocial stress-further characterization and impact of genetic factors. Psychoneuroendocrinology, 2005. 30(2): p. 199-211.

28. Greffrath, W., U. Baumgartner, and R.D. Treede, Peripheral and central components of habituation of heat pain perception and evoked potentials in humans. Pain, 2007. 132(3): p. 301-11.

29. Smith, B.W., et al., Habituation and sensitization to heat and cold pain in women with fibromyalgia and healthy controls. Pain, 2008. 140(3): p. 420-8.

30. Rhudy, J.L., E.J. Bartley, and A.E. Williams, Habituation, sensitization, and emotional valence modulation of pain responses. Pain, 2010. 148(2): p. 320-7.

31. Smith, B.W., et al., The role of resilience and purpose in life in habituation to heat and cold pain. J Pain, 2009. 10(5): p. 493-500.

32. Henchoz, Y., et al., Effects of noxious stimulation and pain expectations on neuromuscular control of the spine in patients with chronic low back pain. Spine J, 2013. 13(10): p. 1263-72.

33. Johnston, V., et al., Alterations in cervical muscle activity in functional and stressful tasks in female office workers with neck pain. Eur J Appl Physiol, 2008. 103(3): p. 253-64.

34. Woda, A., et al., Effect of experimental stress in 2 different pain conditions affecting the facial muscles. J Pain, 2013. 14(5): p. $455-66$

35. Tsai, C.M., et al., Human masticatory muscle activity and jaw position under experimental stress. J Oral Rehabil, 2002. 29(1): p. 44-51.

36. Thombs, B.D., et al., A validation study of the Dutch Childhood Trauma Questionnaire-Short Form: factor structure, reliability, and known-groups validity. Child Abuse Negl, 2009. 33(8): p. 518-23.

37. Bernstein, D.P., et al., Initial reliability and validity of a new retrospective measure of child abuse and neglect. Am Psychiatry, 1994. 151(8): p. 1132-6.

38. Treede, R.D., et al., A classification of chronic pain for ICD-11. Pain, 2015. 156(6): p. 1003-7. 


\section{Samenvatting}

Valorization

Dankwoord

Curriculum Vitae

List of publications

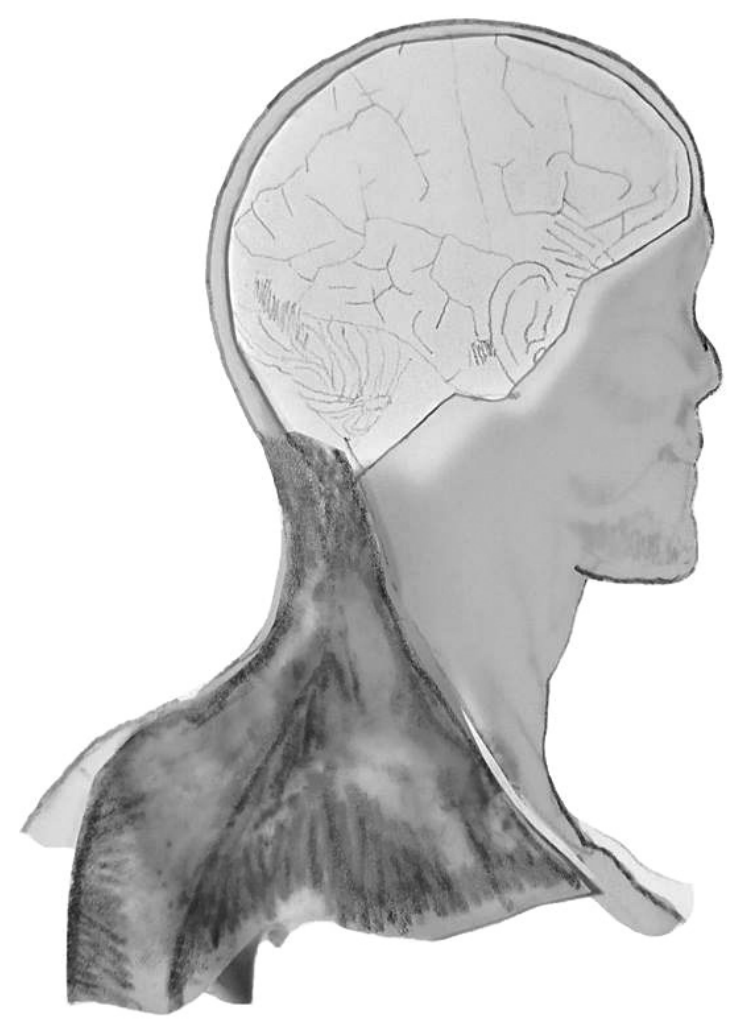


Het doel van het gepresenteerde onderzoek - de basis van het proefschrift dat u in handen heeft - was om psychofysiologische reacties in relatie tot stress en pijn te onderzoeken om zo meer inzicht te krijgen in onderliggende mechanismes. Tevens wilden we vaststellen welke factoren geassocieerd zijn met, of invloed hebben op iemands kwetsbaarheid voor de ontwikkeling van stressgerelateerde gezondheidsproblemen of (chronische) pijn.

Zowel centrale als perifere psychofysiologische parameters zijn onderzocht om deze onderliggende mechanismen te exploreren. De data van de studies die in dit proefschrift gepresenteerd worden, zijn geanalyseerd met behulp van zogeheten 'multilevel regressie analyse'. De psychofysiologische parameters die in dit proefschrift aan bod komen zijn pijn-en stressgerelateerde elektro-encefalografische activiteit (EEG) en elektro-myografische activiteit (EMG): elektrische activiteit welke respectievelijk gegenereerd wordt in het brein of doo spierweefsel. Omdat uit de literatuur gebleken is dat voornamelijk de trapeziusspieren relatief sterk op stress reageren, lag de focus van het onderzoek naar spierreactiviteit ten gevolge van stress juist op deze spieren.

Twee experimentele designs zijn gebruikt om bovenstaande doelstellingen te bereiken Het eerste experiment betrof een nieuw ontworpen stressexperiment. Zowel spieractiviteit als corticale activiteit werd gemeten en onderzocht. Om meer inzicht te krijgen in de biopsychosociale mechanismen van stressreactiviteitwerd bovendien de invloed van een aantal psychologische factoren op de psychofysiologische activiteit gedurende het stressexperiment onderzocht. Naast dit stressexperiment werd ook gebruik gemaakt van een pijn-habituatieexperiment. Het doel van het pijnexperiment was om de psychofysiologische reacties in relatie tot pijn te onderzoeken en om te bekijken wat de invloed is van pijnhypervigilantie (verhoogde aandacht voor pijn) op de spieractiviteit van de trapeziusspieren. Zoals in de literatuur beschreven staat, kunnen concepten als pijn en stress het beste bestudeerd worden binnen een biopsychosociaal raamwerk. Een dergelijk biopsychosociaal model, dat uitgaat van de nauwe relatie die bestaat tussen stress en pijn, is gepresenteerd in de introductie van dit proefschrift.

\section{STRESSREACTIVITEIT EN DE TRAPEZIUSSPIEREN}

Een van de onderzoeksvragen van dit proefschrift betrof het in kaart brengen van de reactiviteit van de trapeziusspieren in stressvolle situaties. In hoofdstuk 2 is het nieuw ontworpen stressparadigma beschreven. Stimuluselektrodes werden op de middelvinger van de linkerhand van de proefpersoon bevestigd. Eerst werd een baselinemeting uitgevoerd. Vervolgens werd in een tijdspanne van 5 minuten één enkele oncontroleerbare en onvoorspelbare pijnlijke prikkel toegediend: proefpersonen werden niet ingelicht over het precieze moment van toediening noch over de intensiteit die de prikkel zou hebben. Op deze manier werd een duale vorm van stress geïntroduceerd. Ten gevolge van het geven van de instructie van het experiment werd er een cognitieve anticipatoire stressfase gecreëerd: de proefpersoon wist dat er onvermijdelijk een pijnlijke prikkel zou volgen. Naast deze vorm van anticipatoire stress werd er een tweede vorm van stress gecreëerd door het toedienen van de pijnlijke prikkel zelf. Op deze manier werd het stressexperiment ingedeeld in verschillende fases: de anticipatoire pre-stimulus fase, een kortdurende directe reactie op de prikkel zelf en de post-stimulus fase, waarin de proefpersoon wist dat er geen pijnlijke prikkel meer zou volgen. Tijdens het gehele experiment werd EMG activiteit gemeten.

De verschillen in gemiddeld niveau van spieractiviteit waren in overeenstemming met met de verwachtingen op basis van stressinductie: het gemiddelde niveau van spieractiviteit was hoger in de pre-stimulus periode in vergelijking met de baseline en de post-stimulus periode. Anticipatoire stress bleek dus geassocieerd met een verhoogd gemiddeld niveau van spierspanning. Het tweede doel van deze studie was om een statistische techniek te introduceren die we tevens bij alle andere studies in dit proefschrift gebruikt hebben: multilevel regressie analyse. Met deze techniek konden lineaire en niet-lineaire tijdeffecten worden aangetoond in de gemeten EMG activiteit gedurende het stressexperiment. De resultaten van de eerste studie laten zien dat het nieuw ontwikkelde stressparadigma valide is en zowel fysieke als cognitieve stress induceert. Er werd duidelijk aangetoond dat de spieractiviteit tijdens het experiment zowel lineair als niet-lineair verandert. Ook waren er aanwijzingen dat de spierreactiviteit beïnvloed werd door het subjectieve stressniveau van de deelnemers.

In hoofdstuk 3 werd EMG stressreactiviteit onderzocht als fysiologische marker in relatie tot traumatische ervaringen in de kindertijd. Deze studie richtte zich enkel op de anticipatoire fase van het stressexperiment. Het aantal geïncludeerde proefpersonen voor deze studie is uitgebreid ten opzichte van de eerste studie. In de literatuur wordt beschreven dat er verschillende stressgerelateerde psychofysiologische variabelen zijn, gemeten op latere leeftijd, die geassocieerd worden met traumatische ervaringen in de kindertijd. Er zijn echter (nog) geen studies die de invloed van deze traumatische ervaringen op spieractiviteit als psychofysiologische stressmaat rapporteren. De resultaten van de studie die beschreven zijn in hoofdstuk 3, tonen een associatie tussen traumatische ervaringen op kinderleeftijd en veranderde spierreactiviteit. Er waren per proefpersoon twee meetmomenten, $t=0$ en $\mathrm{t}=6$ maanden, waarop het stressparadigma op identieke wijze werd uitgevoerd. Er werd geen verschil in effecten geconstateerd tussen beide metingen. Dit suggereert een robuuste, aanhoudende invloed van traumatische ervaringen gedurende de kindertijd op de psychofysiologische activiteit in het latere leven. Hoe hoger de score op de vragenlijst (betreffende traumatische ervaringen), hoe sterker de anticipatoire stressrespons van de 
trapeziusspieren bleek te zijn. Wat opvalt, is dat met name de ervaringen die vroeg in de kindertijd plaatsvonden (tot en met een leeftijd van 11 jaar), gepaard gaan met veranderingen in het EMG. Latere traumatische ervaringen (12 tot en met 17 jaar) lieten dezelfde trend zien, maar de resultaten waren minder prominent en niet altijd significant. Dit is in overeenstemming met de resultaten van eerder onderzoek, die lieten zien dat traumatische gebeurtenissen op vroegere leeftijd meer geassocieerd zijn met problemen wat betreft mentale gezondheid bij volwassenen, in vergelijking met traumatische gebeurtenissen op iets latere leeftijd. Het feit dat negatieve ervaringen in de kindertijd van invloed zijn op de stressreactiviteit van de trapeziusspieren op latere leeftijd, kan een waardevolle bijdrage leveren aan het onderzoek naar de relatie tussen trauma's op kinderleeftijd en de ontwikkeling van diverse stress gerelateerde musculoskeletale aandoeningen.

De bevinding dat er geen verschil was in EMG reactiviteit tussen de eerste en de tweede meting (met een tussenpauze van 6 maanden), versterkt de gedachte dat veranderde spieractiviteit ten gevolge van traumatische gebeurtenissen in de jeugd een blijvende invloed uitoefent. Zo kan beredeneerd worden dat, indien deze veranderde spieractiviteit zou leiden tot een stressgerelateerde aandoening, dit een mogelijkheid tot interventie biedt. Een van de therapeutische mogelijkheden is om myofeedback te geven op de spieractiviteit van de trapeziusspieren. Daarmee kan bewustwording gecreëerd worden over deze verhoogde spieractiviteit en kan de proefpersoon controle krijgen over de verhoogde activiteit. Het meten van de spierreactiviteit middels het ontwikkelde stressparadigma kan ons zowel baseline informatie geven over stressreactiviteit alsook over de effectiviteit van een interventie.

\section{MULTILEVEL MODELING EN PSYCHOFYSIOLOGISCHE UITKOMST-MATEN}

De volgende conclusies kunnen getrokken worden naar aanleiding van hoofdstuk 2 en 3:

De validiteit van het stressexperiment werd aangetoond: de proefpersonen rapporteerden subjectieve stress en, zoals a priori verwacht was, werd de spieractiviteit van de trapeziusspieren beïnvloed door de duale stressor. Onze bevindingen komen overeen met die van diverse andere auteurs die pre-post-verschillen hebben onderzocht. Het is tevens gelukt om aan te tonen dat er sprake is van niet-lineaire tijdeffecten, iets dat bij ons weten met multilevel analyse nog niet eerder onderzocht is.

Datasets met herhaalde metingen, waarbij de herhaalde metingen genest zijn binnen de proefpersoon, zoals gebruikt in alle studies die in dit proefschrift besproken worden, zijn erg geschikt om te onderzoeken met behulp van multilevel analyse. In multilevel analyse wordt rekening gehouden met een dergelijke hiërarchische structuur in de dataset, door het toestaan van residuele componenten op elk level van de hiërarchie. Bovendien laat het gebruik van deze statistische techniek toe dat zowel lineaire als niet-lineaire tijdsverlopen worden onderzocht De fysiologische activiteit van het lichaam is een natuurlijk proces, waardoor beide vormen van tijdeffecten worden verwacht. Ook kunnen zogeheten random intercepten gemodelleerd worden, waardoor er gecorrigeerd kan worden voor interindividuele verschillen

Het is ook aantrekkelijk om deze techniek toe te passen bij onderzoek naar interacties tussen psychologische variabelen en de verandering in psychofysiologische activiteit binnen het experiment. Zo is het bijvoorbeeld een interessant gegeven dat in de eerste studie geen verband werd gevonden tussen het niveau van subjectieve anticipatoire stress en het gemiddelde niveau van spieractiviteit gedurende de verschillende fases van het experiment. Bij het modelleren van kwadratische en inverse tijdeffecten werd er wel een evidente interactie tussen deze tijdeffecten en het niveau van anticipatoire stress aangetoond. De discrepantie tussen deze bevindingen illustreert het belang van het gebruik van multilevel analyse in dit soort onderzoeken: het verkrijgen van additionele en specifiekere informatie die zonder multilevel analyse technieken niet te verkrijgen is. Alle bovenstaande bevindingen benadrukken het belang van het gebruik van geavanceerde statistische technieken als het gaat om het onderzoeken van natuurlijke, (patho)fysiologische processen.

\section{STRESSREACTIVITEIT IN HET BREIN}

Spieractiviteit kan gezien worden als een perifere parameter van stress, terwijl corticale activiteit juist beschouwd kan worden als een centrale parameter. In de studie die beschreven wordt in hoofdstuk 4 analyseerden we de corticale activiteit gemeten gedurende het eerder genoemde stressexperiment. Zowel de pre-stimulus fase als de post-stimulus fase werden gebruikt. Opnieuw werd multilevel analyse toegepast om het beloop van de corticale activiteit (EEG) gedurende het experiment te kunnen onderzoeken.

Met betrekking tot het EEG tijdsbeloop van de alpha activiteit stelden we vast dat de alpha activiteit meer toenam gedurende de post-stimulus periode in vergelijking met de prestimulus periode, conform de a priori hypotheses. Tevens werd aangetoond dat de gamma activiteit in de post-stimulus fase meer afnam in vergelijking met de pre-stimulus periode. Deze effecten werden met name in de frontale, centrale en temporale regionen van het brein geobserveerd. Er werd tevens een toename van activiteit in de trage beta band geobserveerd gedurende de post-stimulusfase in vergelijking met de pre-stimulusfase.

Ook in dit experiment waren wij geïnteresseerd in de interactie tussen psychofysiologische activiteit en psychologische factoren. We gebruikten een gestandaardiseerde vragenlijst met 
betrekking tot de ervaren stress, de Perceived Stress Scale (PSS-10), om te onderzoeken of he tijdsverloop in EEG activiteit wordt beïnvloed door het ervaren stressniveau en het vermogen tot coping. Er werden significante interactie-effecten aangetoond tussen de subschalen van de PSS-10 en de veranderingen in corticale activiteit gedurende het experiment. Een interessante bevinding was dat de interactie-effecten tussen coping en corticale activiteit enerzijds en ervaren stress en corticale activiteit anderzijds erg van elkaar verschilden. Wat betreft de stress-subschaal van de PSS-10 vragenlijst stelden we vast dat een hogere score op ervaren stress werd geassocieerd met een grotere toename van delta en theta activiteit in de post-stimulusfase. De analyses van de coping-subschaal van de PSS-10 lieten juist zien dat een verminderd vermogen tot coping geassocieerd was met een grotere afname in trage beta, snelle beta en gamma activiteit gedurende de post-stimulusfase. Naast deze effecten werden tevens significante effecten in frontale alpha activiteit geobserveerd.

Met de komst van geavanceerde technieken in, zoals bijvoorbeeld fMRI, lijkt het soms alsof elektro-encefalografie in de vergetelheid is geraakt. Het is echter van belang dat de waarde van EEG niet onderschat wordt. Electro-encefalografie is namelijk een relatief goedkope en niet-invasieve techniek, die ons kan voorzien van een schat aan informatie over de temporele werking van het brein. De resultaten die worden beschreven in de proof-of-principle studie in hoofdstuk 4 laten ons zien dat er meer informatie gehaald kan worden uit EEG-activiteit dan vaak wordt verondersteld.

In de literatuur werd alpha activiteit al vaker gelinkt aan stress. De resultaten in hoofdstuk 4 bevestigen deze relatie, maar geven bovendien ook een richting aan het effect. De resultaten stellen ons in de gelegenheid om een natuurlijk tijdsbeloop van de corticale stressreactie te modelleren om op die manier de temporele werking van het brein nog beter te kunnen benaderen. We raden onderzoekers dan ook aan om deze technieken in de toekomst te gebruiken, eventueel in combinatie met aanvullende beeldvormende technieken.

Ten slotte is het boeiend om vast te kunnen stellen dat de invloed van psychologische variabelen geobjectiveerd kan worden. Beide subschalen van de PSS-10 vragenlijst, namelijk de ervaren stress en het vermogen tot coping, bleken een andere invloed te hebben. Hoewel de schalen afgeleiden zijn van dezelfde vragenlijst, is het aannemelijk dat beide schalen gelinkt kunnen worden aan andere 'pathways' in het brein. De uitdaging in dit soort onderzoek is het duiden van de resultaten die gevonden worden, en deze te linken aan verschillende gebieden in het brein. Zoals eerder al gesuggereerd werd, zou het erg interessant en informatief zijn om EEG te combineren met fMRI, om op die manier aanvullende informatie te krijgen over de onderliggende bronnen van de geobserveerde corticale activiteit.

\section{PIJN EN HABITUATIE}

Ten slotte onderzochten we in hoofdstuk 5 de spieractiviteit van de trapeziusspieren van proefpersonen die een pijn-habituatie-experiment ondergingen. Een serie van 25 pijnprikkels werd toegediend, met tussenpozen van ongeveer 10 seconden. Elke prikkel had dezelfde intensiteit, dit wist de proefpersoon niet. Er werden 3 verschillende fases onderscheiden. De pre-stimulus fase betrof 3 seconden voor de prikkel, de pijnreactiviteitsfase betrof 3 seconden na de prikkel en de 3 seconden die daarop volgden werd gedefinieerd als de intermediaire fase. We gebruikten opnieuw multilevel regressie analyse om de gemeten data te analyseren. Tevens werd onderzocht of er een habituatie-effect in de spieractiviteit aantoonbaar was. Naast deze basismodellen onderzochten wij opnieuw of psychologische factoren invloed hadden op (het verloop van) de spieractiviteit van de trapeziusspieren in dit pijnexperiment. De predictor van interesse in deze studie betrof de mate van pijnvigilantie. Wat de anticipatoire fase betrof lieten de resultaten zien dat er sprake was van een lineaire afname in EMG activiteit met vervolgens een exponentiële toename (direct voor toediening van een prikkel). Met behulp van de analyses van de pijnreactiviteitsfase kon worden aangetoond dat er een inverse afname van spieractiviteit was, gevolgd door een lineaire toename.

Met het oog op de algemene tendens over alle 25 stimuli, werd een lineair habituatie effect van spieractiviteit geobserveerd over zowel de linker als de rechter trapezius. Ook werd vastgesteld dat er sprake was van een significante interactie tussen habituatie over de 25 stimuli en het inverse tijdseffect van de spieractiviteit in de pijnreactiviteitsfase. Dit betekent dat er een snellere return to baseline plaatsvindt naarmate het experiment vordert, een fenomeen wat overeenkomt met een natuurlijk fysiologisch reactiepatroon.

Habituatie kan beschouwd worden als een basaal mechanisme, dat mensen toestaat om te adapteren aan repetitieve blootstelling aan (pijnlijke of stressvolle) gebeurtenissen. Verschillende studies hebben de complexiteit van habituatiemechanismen aangetoond. Om zoveel mogelijk mechanistische kennis te vergaren als mogelijk is zijn er nog veel meer studies naar habituatie nodig. Voornamelijk de relatie tussen psychologische factoren en fysiologische habituatie is interessant om uit te werken en te onderzoeken

Als laatste hebben we geprobeerd om een interactie te modelleren tussen fysiologie en psychologische variabelen in een (habituatie)experiment waarin een serie pijnlijke prikkels toegediend werd. Wat betreft de invloed van pijnhypervigilantie bleek dat proefpersonen met hoge scores op pijnvigilantie verhoogde spierreactiviteit laten zien in de anticipatoire fase. Dit is mogelijk het gevolg van een continue staat van aandacht voor pijn. 
Het is aanbevelenswaardig om in toekomstige studies meer onderzoek te doen naar de relatie tussen de habituatie over een serie prikkels, fysiologische (pijn)reactiviteit en pijnrelevante psychologische variabelen. De resultaten beschreven in hoofdstuk 5 zouden bij kunnen dragen aan een theoretisch model betreffende habituatie. Bovendien benadrukken ze de noodzaak voor een multidimensionale aanpak van stress- en pijngerelateerde problemen. Daarnaast kunnen de resultaten leiden tot ontwikkeling van nieuwe en/of aanpassing van bestaande therapeutische interventies. Deze interventies kunnen zich bijvoorbeeld richten op myofeedback of op het aanpassen van pijn- of stressgerelateerde cognities.

\section{BEPERKINGEN VAN HET ONDERZOEK EN SUGGESTIES VOOR} VERBETERING

Het experimentele design zoals gebruikt in dit proefschrift heeft een aantal beperkingen, welke reeds besproken zijn in elk hoofdstuk apart. Er zijn echter ook een aantal algemene beperkingen welke in de volgende sectie worden besproken.

Wat betreft de EMG-gerelateerde studies: voor aanvang van de studies werd besloten om de spieractiviteit van de trapeziusspieren te meten, omdat na een literatuurstudie deze het meest stressgevoelig bleken. Het is echter heel aannemelijk dat de spieractiviteit van de trapeziusspieren gedurende een stress- of pijnexperiment andere informatie toont dan bijvoorbeeld de nekspieren, de paraspinale spieren of de spieren van het gezicht. Voor toekomstig onderzoek zou overwogen kunnen worden om ook de spieractiviteit van bovengenoemde spieren te meten.

Wat betreft de statistische technieken die wij gebruikt hebben voor het analyseren van de data is het belangrijk om te vermelden dat de techniek (soft- en hardware) ons op dit moment nog niet toelaat om een ongelimiteerd aantal segmenten van gemeten EMG activiteit te analyseren. Daarom moest er een balans gezocht worden tussen het aantal segmenten dat softwarematig te modelleren is enerzijds en de lengte van elk segment anderzijds. Als je bijvoorbeeld kiest voor korte segmenten (korter dan 250ms) wordt het aantal segmenten om te analyseren bijgevolg zo groot dat het met de huidige hardware onmogelijk is het gewenste model uit te voeren.

Onderzoekers zouden verder kunnen kiezen voor het includeren van andere stressgerelateerde variabelen,zoals cortisollevel in speeksel of serum, om de impactvanzowel het stressexperiment als het pijnexperiment verder te valideren en te exploreren. Om de betrouwbaarheid van bepaalde bevindingen te vergroten zou men kunnen overwegen om aanvullende vragenlijsten te includeren, zoals bijvoorbeeld de Childhood Trauma Questionnaire.

\section{BACK TO THE FUTURE}

Wat betreft de gepresenteerde (nieuwe) bevindingen in dit proefschrift, is het belangrijk om te benoemen dat repliceerbaarheid noodzakelijk is, zoals voor ieder ander onderzoek. Ook zijn er een aantal zaken die nog niet onderzocht zijn. In het kader van toekomstig onderzoek kan een waaier aan onderzoeksvragen nog worden opgesteld. In de volgende paragrafen wordt hierop ingegaan.

\section{Klinische populatie}

Om de klinische toepasbaarheid van de huidige resultaten en conclusies naar aanleiding van dit onderzoek te vergroten is aanvullend onderzoek in specifieke klinische populaties nodig. In dit proefschrift werden, in een gezonde populatie, de modulerende effecten onderzocht van subjectieve stress, pijnvigilantie en traumatische ervaringen op kindertijd op psychofysiologische maten. Deze lijn van onderzoek voortzettend, zou men in de toekomst kunnen focussen op proefpersonen die te maken hebben met stressgerelateerde ziektes, zoals post-traumatische stress syndromen, op chronische pijnpatiënten of op een klinische populatie van mensen die traumatische ervaringen op de kinderleeftijd hebben ondervonden.

In hoofdstuk 5 van dit proefschrift hebben we gebruik gemaakt van een pijnvrije subgroep. Een groot deel van onze totale studiepopulatie rapporteerde ofwel acute ofwel chronische pijn, maar er werd een grote verscheidenheid aan klachten tentoongespreid. Voor toekomstig onderzoek lijkt een populatie van patiënten die lijden aan nek- en schouderklachten geschikt. Het zou bijvoorbeeld interessant zijn om het contrast te onderzoeken tussen proefpersonen met acute pijn en gezonde controles enerzijds en proefpersonen met chronische pijn (klachten langer dan 3 maanden) en gezonde controles anderzijds.

\section{Opties voor interventie}

In het kader van therapeutische opties zou het de moeite waard zijn om experimenteel te interveniëren in de eerdergenoemde klinische populaties. Zo zou een klinische populatie van patiënten met chronische pijn gerandomiseerd kunnen worden in 2 groepen. Bij deze 2 groepen zou in een baselinemeting de psychofysiologische stress- en pijnreactiviteit gemeten kunnen worden, om vervolgens myofeedback als therapeutische interventie toe te passen. $\mathrm{Na}$ 6 maanden is het mogelijk om het effect van de interventie te objectiveren door opnieuw de vragenlijsten af te nemen en het experimentele protocol uit te voeren. Ten slotte is het te overwegen om een dergelijke therapie in de vorm van een dubbelblinde randomized clinical trial uit te voeren (gebruikmakend van sham-myofeedback). 


\section{Subgroepen}

Een bijkomstig aspect zou kunnen zijn dat er gefocust wordt op meer specifieke subgroepen bij de al eerder genoemde klinische populaties. Zo werd bijvoorbeeld in hoofdstuk 5 een interactie tussen pijnhypervigilantie en psychofysiologische pijnreactiviteit aangetoond. Bovengenoemd voorgesteld paradigma, waarbij je een groep proefpersonen met acute pijn vergelijkt met een groep gezonde controles, zou de onderzoekspopulatie onderverdeeld kunnen worden in subgroepen wat betreft pijnvigilantie. Zo zouden vier verschillende groepen vergeleken kunnen worden: gezonde controles, die ofwel hoog ofwel laag scoren op pijnhypervigilantie en proefpersonen met acute pijn, die ook hoog of laag scoren op pijnhypervigilantie. Op die manier kan de impact van de mate van pijnvigilantie onderzocht worden en onderliggende mechanismen gedestilleerd worden.

Op een zelfde manier zou een populatie proefpersonen met traumatische ervaringen op kinderleeftijd onderverdeeld kunnen worden in twee groepen, namelijk in een groep die geen (ernstige) klachten meer ervaart van deze trauma's en een groep die nog steeds de nadelige gevolgen ondervindt. Om het contrast tussen deze twee groepen goed te kunnen begrijpen zou ervoor gekozen kunnen worden om een derde groep met gezonde controles toe te voegen.

\section{Prospectieve studies}

Naast de genoemde cross-sectionele studies die eerder voorgesteld werden, zou een prospectieve studie ons in staat stellen om de ontwikkeling van bepaalde fenomenen te onderzoeken en kan het ons additionele informatie bieden. Bij het onderzoeken van een acute pijnpopulatie in een prospectief design is het mogelijk de ontwikkeling van acute naar chronische klachten in kaart te brengen. De link tussen veranderende psychofysiologische reactiviteit, psychologische factoren en de translatie naar eventuele chroniciteit kan op die manier onderzocht worden.

\section{SLOTCONCLUSIE}

Het belangrijkste doel van dit proefschrift was om de psychofysiologische activiteit die gepaard gaat met stress en pijn te onderzoeken en om de interactie met psychologische variabelen in kaart te brengen. De resultaten hebben ons laten zien dat zowel traumatische gebeurtenissen op de kinderleeftijd, mate van ervaren stress en coping alsook pijnhypervigilantie van invloed zijn op de psychofysiologische activiteit in een setting waar stress en pijn experimenteel worden gemanipuleerd. De resultaten dragen bij aan een toenemend inzicht in de (complexe) onderliggende mechanismen van de constructen pijn en stress. De in dit proefschrift ontwikkelde paradigmata voor pijn- en stressreactiviteit stellen ons in staat om kwetsbaarheidsfactoren te kunnen detecteren. De resultaten onderstrepen het belang van een biopsychosociale benadering van pijn- en stressgerelateerde klachten. De uitdaging is om deze kennis te integreren in preventieve interventies teneinde chroniciteit van klachten te voorkomen, alsmede in de optimalisatie van curatieve behandelingen. 
Samenvatting

Valorization

Dankwoord

Curriculum Vitae

List of publications

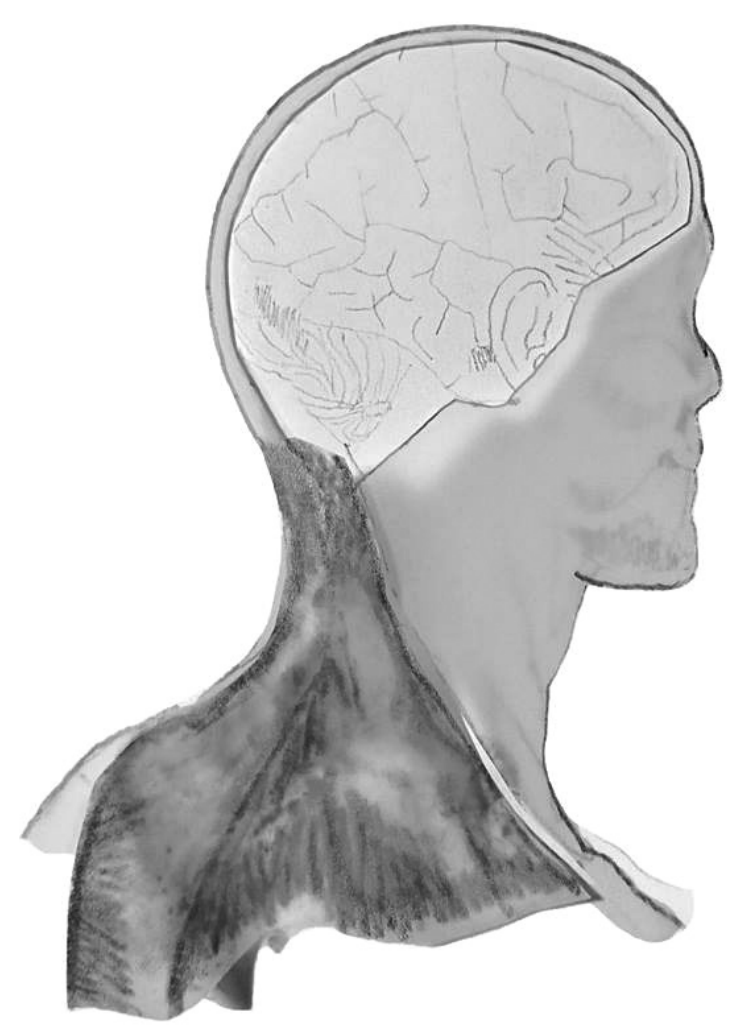


All research leads to new conclusions, no matter how large or small the findings are, and the results can lead us in new directions to further elaborate the topic. The aim of this thesis was to examine the concepts of stress and pain by exploring the effects on psychophysiological measures. The results of the research presented here have yielded new insights in the underlying mechanisms of stress and pain, and more specifically, provided new information on interactions between psychological factors and pain- and stress-related psychophysiological activity. All results are discussed in the corresponding chapters. Broader clinical implication are reflected on in the general discussion chapter. Here, the implications of this thesis are discussed in an extensive context, and a more general view, in order to expound the societal relevance of the research presented in this thesis.

\section{SOCIETAL RELEVANCE OF THIS THESIS}

As mentioned earlier, pain and stress are common phenomena to all of us. Alongside commonly experienced daily hassles, many of us also experience (serious) adverse life events, either in childhood or later in life, which can have an enormous impact. Acute pain and acute stress are essential in our lives, allowing us to survive. When it comes to long-lasting or recurrent pain, however, the impact on the subject as well as the psychosocial circumstances (personality, work, family, society etc.) may create the perfect scenario for progression into chronicity. In this case, pain seems not to serve any goal. Prolonged exposure to stress makes us vulnerable and predisposes us to mental disorder and stress-related health problems.

The prevalence of chronic pain, chronic pain syndromes, prolonged exposure to stress and stress-related health problems is high in modern society. The economic burden is therefore extensive. Over and above the medical costs, a large share of the economic burden is caused by non-medical costs, such as work absenteeism and unemployment benefits. Furthermore, it creates personal suffering, which influences both the subject as well as his or her environment. The results presented in this thesis reflect the inter-individual differences in experience of stress and pain on a psychophysiological level. They suggest the possibility to broaden the personalization of medicine by offering possible tailor-made interventions. The paradigms used in this thesis, concerning both stress and pain reactivity, allow us to detect risk factors that increase vulnerability. If these risk factors can be distilled, interventions could be applied where needed. For example, it would be interesting to screen for childhood adversities during the treatment of mental illnesses or stress-related illnesses. When identified, interventions, such as myofeedback or cognitive behavioral interventions, can be implemented. Applied in such way, these interventions can be fast and cost-effective, and can increase the prognostic values. The present research can be viewed as the first steps toward clinical appliance.

\section{INNOVATIVE TECHNIQUES}

With ongoing innovation in IT, some former limitations are no longer an issue. Even during the past few years, while conducting this research, improvements in statistical software have allowed us to use better fitting statistical techniques. Additionally, advances in computer hardware have enabled us to perform more sophisticated statistical analyses. Researchers should exploit these new possibilities and choose the best fitting statistical technique to examine their data, to approach the essence of the matter of interest. In all studies presented in this thesis, multilevel random regression analysis was used in order to unravel the complex structure of the data. The results showed the benefit of using this advanced statistical technique presenting additional and more specific information in comparison with conventiona techniques. Multilevel analysis techniques offer great potential in the examination of natural psychophysiological processes, both at group level as well as at the level of the individual.

\section{FUTURE DIRECTIONS}

Although the population of interest for this thesis was a (relatively) healthy, general population, without any diagnosed illnesses/diseases, this research has nevertheless provided evidence that interaction effects on psychophysiological measures are present, for both stress-related diseases and pain-related problems. The research presented in this thesis can therefore be viewed as a proof of principle. In future research, clinical populations can also be included, providing more in-depth insight into the constructs of stress and pain and, above all, into transition processes of chronicity. Populations of interest are patients with chronic pain and patients with stress-related diseases, such as PTSS and fibromyalgia. Also, this proof of principle can be used in the examination of neck pain, possibly resulting from computer usage, smartphones etc. Furthermore, although the focus of the present research was the trapezius muscles, the techniques used can be applied in the examination of the activity of various muscles or muscle groups. Thus, other clinical populations can become the subject of interest, e.g. populations with chronic back pain, orofacial pain, headaches etc. In conclusion, foundations have been laid for the use of a new experimental paradigm, including a multilevel analysis protocol, enabling the examination of diverse populations and specific problems. 
Samenvatting

Valorization

Dankwoord

Curriculum Vitae

List of publications

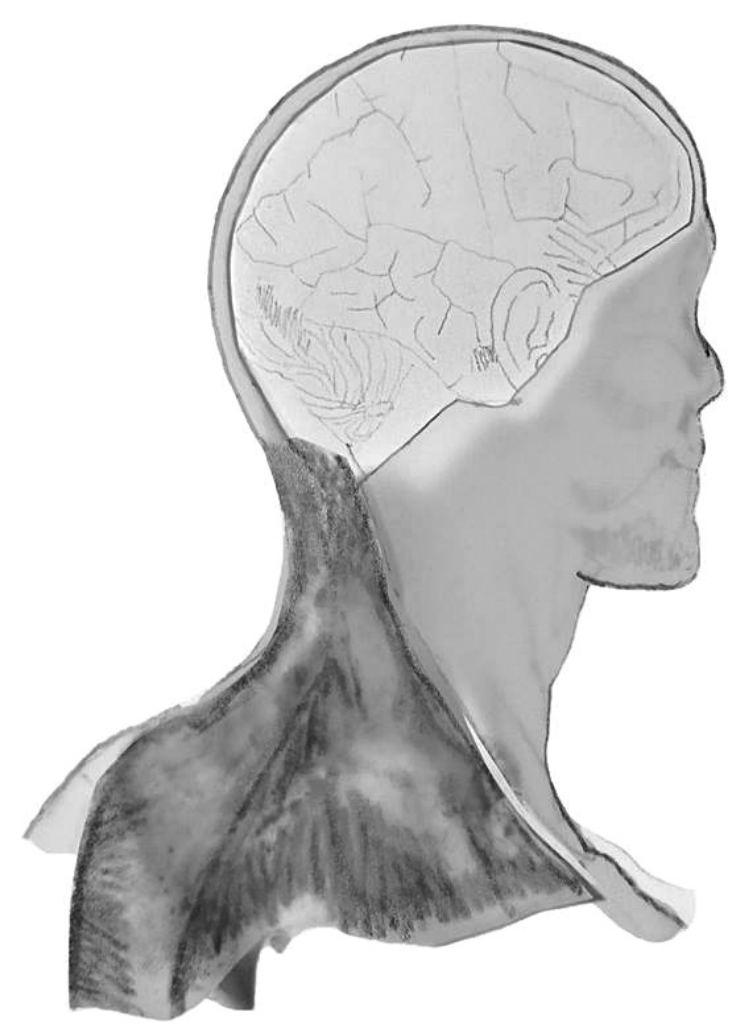


Het dankwoord is het moeilijkste hoofdstuk van het hele proefschrift, zo werd mij op het hart gedrukt. Toch heb ik geprobeerd dit hoofdstuk in een wat rapper tempo te schrijven dan mijn artikelen. Gelukkig had ik het zelf al jong in de gaten: ik wilde een boek schrijven. Talloze malen begonnen, nooit verder gekomen dan een aantal zinnen op de eerste pagina. Inmiddels zijn we meer dan 15 jaar verder en heb ik deze keer wel het doorzettingsvermogen gehad om het af te maken. Het feit dat jullie een boekje voor je neus hebben met ondergetekende als auteur ligt uiteraard niet alleen aan mezelf.

Ik zou nog veel meer mensen moeten bedanken, maar de publicatiekosten gaan per pagina dus ik moest wat keuzes maken. Niet getreurd, als je er niet in staat betekent dat niet dat ik je niet waardeer!

Allereerst, zonder de goede begeleiding van mijn promotieteam en de steun van mijn (onderzoeks)collega's was hier natuurlijk helemaal niets van terecht gekomen. Dus, vele malen dank, allen!

Richel, ik ben nog niemand tegen gekomen die zo enthousiast is over elektrofysiologie, over statistische methoden en over het doen van onderzoek. Op momenten dat ik er niet zoveel zin meer in had was dat erg handig en noodzakelijk. Met name het doorworstelen van dagen vol statistische analyses had ik niet gered zonder jouw hulp. Dank voor de positieve woorden en de peptalks op z'n tijd, en voor je kennis en kunde. De beste tip die ik van je gekregen heb is sowieso dat áls ik te hard rijd, dat ik dan beter zo dicht mogelijk op m'n voorganger ga rijden, zodat ik niet geflitst kan worden. Bij deze: 1) bumperkleven noemen we dat, dat is net zoals te hard rijden niet geaccepteerd op de Nederlandse wegen. 2) Ze flitsen juist vaak aan de achterkant, dus die vlieger gaat niet op! Gelukkig is Birgit degene die jullie auto bestuurt, en meestal rijdt ze alleen maar te hard in Duitsland. Birgit, bedankt voor alle goede zorgen, de thee, en de betrokkenheid!

Jim, ik blijf onder de indruk van de snelheid waarmee mijn teksten retour komen, van je altijd scherpe blik en het gemak waarmee je dit alles lijkt te doen. Dank voor je nooit aflatende positiviteit. De volledig rood gemaakte tekst met allerlei aanpassingen en suggesties worden altijd voorzien van een positieve kreet (brilliant, well done, looking better all the time, excellent) De variant 'dat is echt dynamite' is mijn persoonlijke favoriet. Het werkt aanstekelijk! Dank voor alles! BW, R.

Hermie, mijn tweede promotor. Ook aan jou dank voor je expertise, je vrolijkheid en enthousiasme! De afstand Maastricht-Enschede is behoorlijk, gelukkig zijn wij al tevreden met een ontbijt-overleg in de Beuningse McDonalds. Ondanks je drukke agenda nam je toch tijd voor me en had je zin om energie te steken in dit project. Fantastisch!

Carine, mijn andere tweede. Dank voor je scherpe commentaar en je tips, jouw kritische blik heeft me telkens stappen vooruit geholpen. Ik bewonder je doorzettingsvermogen en je enthousiasme voor onderzoek en werk. On the way heb je me voorzien van goede en zeer waardevolle adviezen; wie weet wat voor toekomst wij samen nog zullen hebben. Lonneke toen ik voor het eerst in Vijverdal kwam was je daar al. Altijd aanpakken en doorzetten. Ondertussen al bijna psychiater, dat kan alleen maar positief uitpakken. Dank voor de gezelligheid, de wijntjes op z'n tijd en je positieve instelling; ik ben trots op je! Suzanne, helaas ben jij al weer een tijdje vertrokken naar het noorden des lands. Gelukkig hebben we niet al te veel tijd fulltime samen in Vijverdal doorgebracht, anders stond ik hier zeker pas een jaar ater. Wel altijd gezellig, niet zo efficiënt. Dankzij jou heb ik heel wat koopappartementen in Groningen gezien. Je hebt het fantastisch gedaan de afgelopen jaren en gaat sowieso een tophuisarts worden. Elaine, net als het voltooien van een triatlon ga je als een gek met analyseren en schrijven. Zoals je ziet, uiteindelijk resulteert het werk in een proefschrift. Veel succes met alles! Marga, zonder jou was dit proefschrift er zeker niet geweest. Als ik alle dataverzameling zelf had moeten doen, was ik nog lang niet klaar geweest. Je hebt het super gedaan! Veel succes met alles in de toekomst.

Dank aan alle collega's in Vijverdal, voor de vriendelijkheid en de hulp hier en daar. In het bijzonder: Sanne, dank voor alle tips \& tricks! Ron, fijn dat je deur altijd open staat, dat je altijd mee wil denken, en dat je alles probeert te doen om gecrashte bestanden te redden als ik in paniek aan je bureau sta.

Dank aan alle studenten die geholpen hebben met de dataverzameling.

Ik wil graag de leescommissie, en in het bijzonder Frenk, bedanken voor hun tijd en interesse.

En natuurlijk, dank aan alle proefpersonen. Zonder jullie kan de wetenschap niet verder komen.

Now is the time om mensen in het zonnetje te zetten die niet veel met het onderzoek an sich te maken hebben gehad, maar daardoor niet minder belangrijk zijn geweest in de totstandkoming van dit proefschrift. Want met hier en daar een bemoedigend woord gezelligheid, wijn, John Tinic's, etentjes en feestjes is het leven van een promovendus weer een stukje leuker!

Allereerst mijn collega's van het SJG: ik kan me geen betere werkplek bedenken, zeker voor mijn eerste baan. Als het kon zou ik nog heel lang blijven. Helaas moet ik toch nog wel ergens 
een opleiding vandaan toveren. Wie weet kruisen onze paden opnieuw, ik zou het heel erg leuk vinden! Dank voor de gezelligheid, het meeleven en de steun (waarbij sorry voor mijn gezeur de laatste tijd)! Een speciale vermelding kun je helaas niet afkopen, trouwens, maar ik draag jullie allemaal een warm hart toe.

Met name de nuchtere woorden van mijn ouders ('Tja, je bent er zelf aan begonnen'; of 'Je bent zeker weer heel moe') hebben bijgedragen aan het realiseren van dit proefschrift. De gezelligheid in de weekenden (of tegenwoordig ook wel doordeweekse dagen) thuis dragen altijd bij aan mijn innerlijke rust: wandelen met Caspertje*, René* (ja, dat is een hondennaam) en nu Floortje of Sam terwijl ons moeder ongevraagd blijft doortetteren (of ben ik degene die altijd zoveel praat?). Lekker buiten, op het speciaal voor mij aangelegde gazon. Wat ook bijdraagt aan mijn rust is een citytrip naar Rome, flaneren en mojito's drinken; als de zigeuners je portemonnee maar met rust laten, dan kun je de functie van je stok beperken tot hulpmidde in plaats van wapen, papa. Laten we maar snel weer een andere stad gaan bezoeken.

Naast mijn ouders wil ik uiteraard mijn broers en zus bedanken. Als we met z'n allen aan tafe zitten laten we elkaar altijd rustig uitpraten en praat niemand er ooit doorheen. Jullie zijn dan ook tot in de puntjes op de hoogte van mijn onderzoek... Gelukkig horen we allemaal de belangrijkste dingen van elkaar, daar gaat het om.

Jan, de oudste van vier. Ik vind het heel fijn dat je er altijd bent op de belangrijke momenten, daar hoef ik nooit over te twijfelen. Ik bewonder je om je enthousiasme, je loyaliteit en je oprechtheid. Met je nieuwste aanstelling zal je het nog druk krijgen de komende jaren, maar ik hoop dat je nog wat tijd over hebt om je eigen dromen na te jagen. Als er geen leuke boerendochter beschikbaar is in Zeeuws-Vlaanderen, moet je het Limburgse heuvelland eens overwegen, dat zou gezellig zijn!

Liselot, de kleinste van vier. Altijd gezellig als jij er bij bent, telefoongesprekken duren volgen George standaard te lang. Gelukkig werken we allebei in de zorg, zo kunnen we de leukste verhalen uitwisselen en mensen diagnosticeren met vreselijke ziektes. Maar het leven bestaat niet alleen maar uit werk (zeker afgelopen jaar niet)! Gelukkig ben je Valentyn tegengekomen en ik ben blij dat ik getuige mocht zijn bij jullie trouw. Zo'n vangst moet je niet laten gaan, zeker niet gezien zijn talent voor koken. Heel veel geluk met elkaar en de kleine Feline, ik ben een heeeele trotse tante!

Jeroen, de luidste van vier. Gelukkig zien wij elkaar af en toe als je weer eens aanmeert in Maastricht en omstreken! Ik moet het zeggen: knap gedaan, de afgelopen jaren. Komt helemaal goed met jou en ik ben benieuwd welke avonturen jullie gaan meemaken met jullie prachtige zeilboot. Gelukkig heb je Rachel altijd aan je zijde, dan hoef ik me niet ongerust te maken ;-). Geniet van het leven (dat hoef ik jullie niet te vertellen natuurlijk)! Natuurlijk mag ook Ozzy niet ontbreken: bedankt om zo prominent aanwezig te zijn.

Henri en Ellen, dank voor de kritische vragen en de oprechte interesse. Jullie staan altijd voor ons klaar, en zonder jullie zolder had ik al menig carnaval (of themafeesten, werkuitjes, noem maar op) zonder pekske rond moeten lopen. Tevens dank voor een continue aanvoer van wasmiddel, wc-papier en zuurvlees, dat mag ook wel eens gezegd worden! Met jullie kreeg ik er nog een heel deel familie bij, langs beide kanten, waardoor ik me ook elk jaar weer kan verheugen op onze gezellige Kerstviering. Jan en Nicole, Koen, Sharon, Wouter, Samira, Janneke, Sander en die lieve kleine Lena: jullie zijn toppers! Zo word ik hier en daar getrakteerd op skilessen, barbecues en tussendoor natuurlijk David Bowie sing-a-longs. Luc ook voor jou een speciale vermelding, zeker gezien het feit dat ik zonder jou alles in Maastricht met de benenwagen zou moeten doen. Bedankt voor alle lieve kaartjes en het feit dat je altijd aan ons denkt.

Anne en El, mijn paranimfen. Ik ben heel blij en opgelucht dat jullie me bijstaan vandaag. Val, hoewel je er fysiek niet bij bent, zullen we zeker aan je denken, want samen zijn we natuurlijk een mooi kwartet. Het eerste jaar in Antwerpen heeft me studie-gerelateerd niet zoveel gebracht (behoudens het knutselen met plastic biomoleculen, ratten dissecteren en de verplichte colleges om half 9 's ochtends waar we al dan niet nuchter aanwezig waren, altijd onder het genot van een kopje thee), maar ik zou júllie voor geen goud willen missen. Ik bewonder jullie alle drie en ben blij dat we met $z^{\prime} n$ allen zoveel moeite doen om elkaar te blijven zien.

Koen, wat begon bij de practica chemie aan de UA, gevolgd door een pannenkoekenfeest ter ere van mijn geplakte fietsband, zette zich de afgelopen jaren gewoon voort. We draaien onze hand niet om voor een dagje Boedapest, Noord-Scharwoude of het 3-landenpunt. Tegenwoordig wat minder vaak, maar daarom niet minder leuk!

Tessie, we zouden elkaar wat vaker moeten zien, maar ondanks af en toe een lange pauze ben je toch een constante factor, gelukkig. Lot \& Pieter, nog steeds zoeken we elkaar op, en daar kan ik alleen maar blij om zijn. Keshia (\&Philip, Lynn en Lauren), mijn Koewachts front. Als ik jullie was, zou ik me als de wiedeweerga eens naar Maastricht bewegen! De planning is namelijk echt om hier nog wel even te blijven hangen. Rianneke, eerst samen in de peuterklas, nu ook in Maastricht! 
Paula, Lisa \& Anouk, mijn matties van de Parkweg. Een groot deel van mijn proefschrift heb ik natuurlijk daar geschreven. Paula, bedankt voor je warrigheid, je gezelligheid en watercrises op z'n tijd. Je doorzettingsvermogen is ongekend, meester! Anouk, de netste, de jongste, de sportiefste mag ik ook wel zeggen. Altijd aan het rennen, lachen en feesten, en dan weer studeren om 8400 's ochtends. Als ik dat soort discipline had gehad... Lisa, meestal was je op stage zodat wij een schare aan huisgenootjes voorbij hebben zien komen (meestal positief...) Gelukkig was je er tussendoor altijd, in je oranje kamer. Bedankt voor je grappigheid, je loyaliteit, je nuchtere blik en je rare theegewoontes. Dat we elkaar nog maar vaak mogen opzoeken!

Dank aan mijn medegeneesco's voor de gezellige BOBjes hier en daar, voor de etentjes en de huisfeestjes. Frank, dank voor de muzikale verrijking van mijn leven. En ook voor de gezelligheid, je enthousiasme en het feit dat je zo in jezelf en anderen gelooft.

Sophia, dank voor het maken van de cover!

Sonja, al die keren dat ik nog snel ff moest studeren in de auto hebben sowieso bijgedragen aan het feit dat ik hier nu sta.

Charlotte W., dank voor het redden op de valreep!

Sistafriends, Annelie, Marion, Mirjam, Niki, Sofie: hoewel de helft alvast gaan zwerven is, blijft de andere helft nog eventjes in het Limburgse land, gelukkig. Bedankt voor alle avonden/ weekendjes/vakanties vol gezelligheid, voor de themataarten of zelfgemaakte mojito's op z'n tijd. Bedankt voor het samen sporten, of samen besluiten dat wijn drinken toch echt gepaster is dan sporten op dat moment, voor het gezellig maken van The Lodge, voor de slaapadresjes in Haarlem, Boxmeer, Zwartsluis, camping Kloosterzande en uiteraard op de Koewacht. Jeroen, geen dank dat wij je aan Annelie hebben gekoppeld. We zagen het al lang aankomen k zou het wel prettig gevonden hebben als jullie als dank de kip toch naar mij vernoemd hadden (voordat ie de dood vond en begraven werd in de groenbak). Helaas, gemiste kans. Sander, goeie keuze om met Niki te trouwen! Ruben, goed dat je Sofie weer terugbrengt naar Maastricht.

Aan alle meeloontjes en banana's! Bas, Bram, Bram B., Martijn, Max, Philippe, Roel, Rolf, Tom, Charlotte, Elles, Ilse, Janina, Kim, Laurie, Stephanie, Veronika: ik heb me vanaf het eerste moment welkom gevoeld. Ik geniet van de etentjes, feestjes, concerten, weekendjes, Weerwolven (deze is voor jou, Bas), babyshowers (van wie niet eigenlijk?), housewarmings, carnavalsdagen/-avonden/-nachten (ik ken alle teksten al bijna even goed als Janina), oudejaarsavonden etc. Mooi hoe iedereen elkaar steunt, zowel in goede als in mindere tijden.
George, a.k.a. Geo, prins Gio, dr. Montiforti, allerliefje. De Parkweg heeft ons heel wat gebracht! Zonder jou zou ik namelijk altijd onder de blauwe plekken zitten van hier en daar wat duizendpoten, spinnen, zilvervisjes en ontelbaar veel bananenboordermotten. Dank om alle zichtbare insecten uit mijn blikveld te verwijderen. Zonder jou zou mijn habitat wat meer op een bescheiden vuilnisbelt gaan lijken, dus dank dat je altijd mijn kleren opvouwt (maar de chaos op mijn bureau gedoogt; ik wed voor 20 euro dat ik het komende week allemaal opruim). Zonder jou was ik nooit winnaar van de Mega ultra super heavy quiz night geworden waarvan de beker godzijdank niet meer in ons appartement staat. Dank voor de cd's van Febries, en misschien ook wel alle andere muzikale bijdrages. Jouw nuchtere kijk op zaken en het aansporen om hier en daar toch maar weer eens wat aan m'n onderzoek te doen heeft de zaken sowieso versneld, zeker de momenten dat je me de bank afduwt als ik in slaap dreig te vallen. Dank om af en toe mijn paniekaanvallen te coveren. Al met al: je bent een topper Gelukkig hebben we de afgelopen jaren met name veel plezier gehad, dus laten we daar nu gewoon maar weer mee verder gaan! 
Samenvatting

Valorization

Dankwoord

\section{Curriculum Vitae}

List of publications

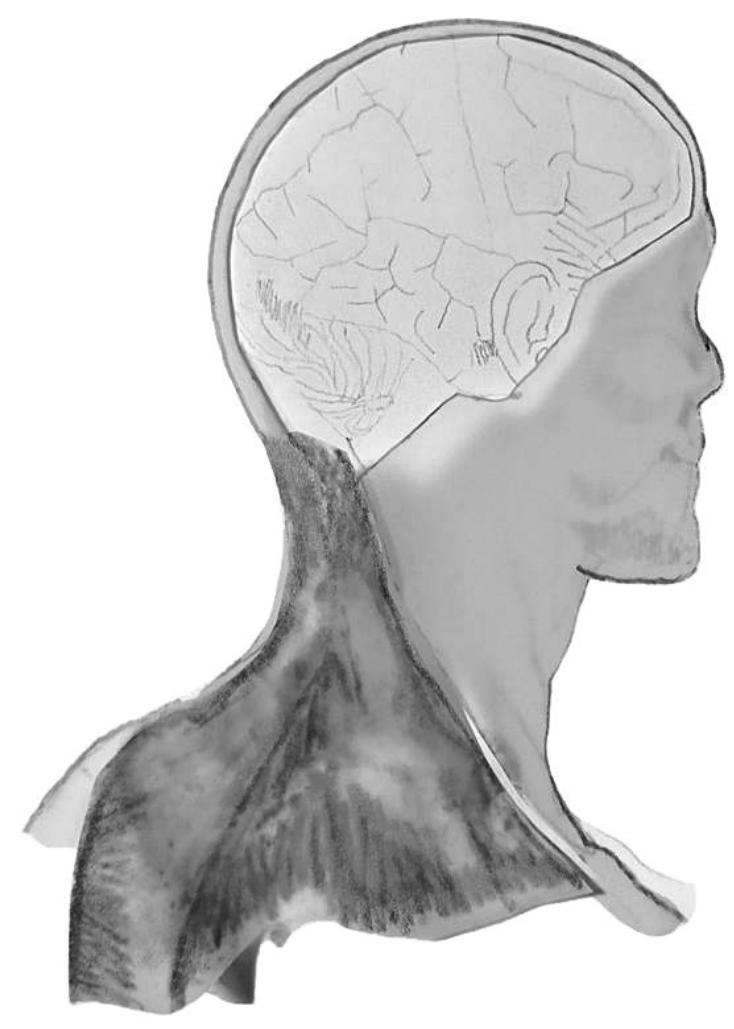


Rosan Luijcks was born on the $13^{\text {th }}$ of April, 1990 in Clinge, Zeeuws-Vlaanderen. She grew up in Koewacht and attended high school at the (Onze Lieve Vrouwe) Presentatie in Sint-Niklaas (Belgium) from 2002 to 2008. After spending a year in biomedical sciences at the University of Antwerp (Belgium), she went for a short period of time to Amsterdam, only to transfer to Maastricht University in October 2009 to receive her medical training. She started her PhDtraining in 2012 and concurrently continued her medical training. She graduated in 2015 and began working at the Emergency Department at Sint-Jans Gasthuis in Weert. She completed her PhD-training, which resulted in the present thesis. 
Samenvatting

Valorization

Dankwoord

Curriculum Vitae

List of publications

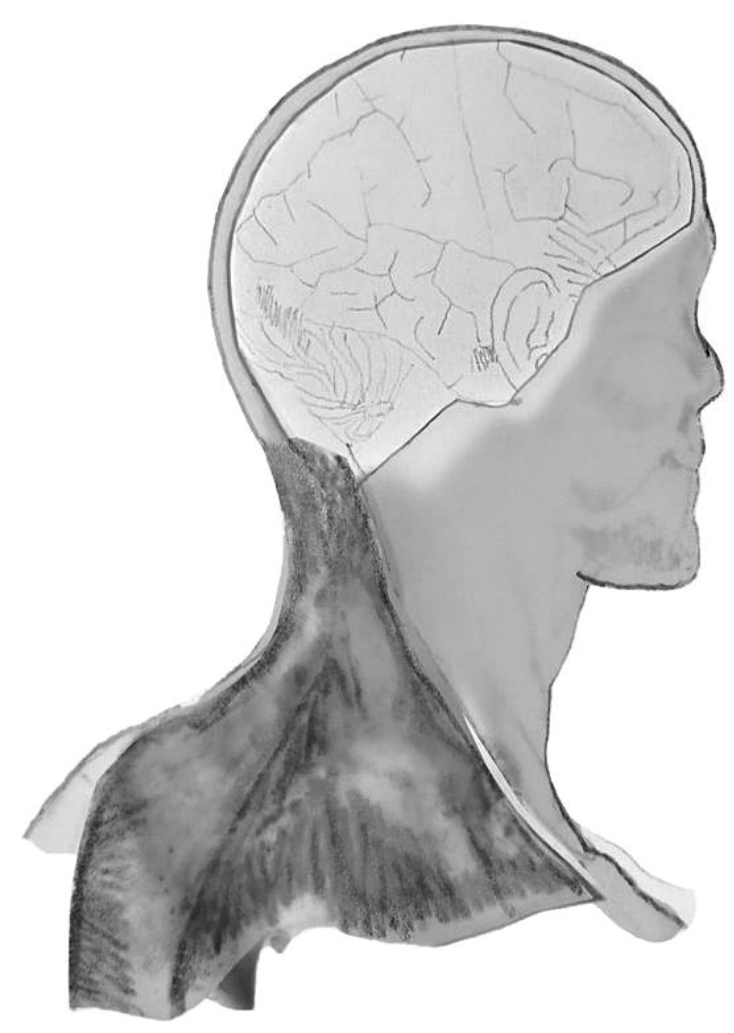


Luijcks R, Hermens HJ, Bodar L, Vossen CJ, Os J and Lousberg R. Experimentally induced stress validated by EMG activity. PLoS One. 2014;9(4):e95215.

Luijcks R, Vossen CJ, Hermens HJ, van Os J and Lousberg R. The Influence of Perceived Stress on Cortical Reactivity: A Proof-Of-Principle Study. PLoS One, 2015. 10(6): p. e0129220

Luijcks R, Vossen CJ, Roggeveen S, van Os J, Hermens HJ and Lousberg R. Impact of childhood adversity on EMG stress reactivity of the trapezius muscle [accepted for publication]

Luijcks R, Vossen CJ, van Os J, Hermens HJ and Lousberg R. Processing pain: The modifying role of hypervigilance on trapezius muscle activity in a pain experiment. [submitted]

Roggeveen S, van Os J, Viechtbauer W, van Alphen A, Ronner J, Luijcks R and Lousberg R. The brain exposed to remote controlled alternating mobile phone conditions: what happens to the EEG? [submitted]

Vossen CJ, Luijcks R, Joosten EAJ, van Os J and Lousberg R. The Influence of Pain Hypervigilance on Cortical Processing and Habituation to Pain in Healthy Subjects: A cross-sectional pain ERP study. [submitted] 
